\title{
Modeling of Magnetic Spintronic Devices: From Theory to Applications
}

\footnotetext{
A Dissertation

Presented to

the Faculty of the School of Engineering and Applied Science

University of Virginia

In Partial Fulfillment

of the requirements for the Degree

Doctor of Philosophy (Electrical and Computer Engineering)

by

Mehdi Kabir

May 2017
} 
(c) Copyright by Mehdi Kabir

All rights reserved

May 2017 


\section{Approval Sheet}

This dissertation is submitted in partial fulfillment of the requirements for the degree of Doctor of Philosophy (Electrical and Computer Engineering)

\section{Mehdi Kabir}

Mehdi Kabir

This dissertation has been read and approved by the Examining Committee:

Mircea R. Stan

Mircea R. Stan, Advisor

Lloyd Harriott

Lloyd R. Harriott, Committee Chair

Jiwei Lu

Jiwei Lu (MSE)

Robert M. Weikle

Robert M. Weikle (ECE)

Arthur W. Lichtenberger

Arthur W. Lichtenberger (ECE)

Accepted for the School of Engineering and Applied Science:

\section{Craig Benson}

Craig Benson, Dean, School of Engineering and Applied Science

May 2017 


\section{Abstract}

In recent years, there has been a paradigm shift ushered in by More-than-Moore technologies which has focused on functional diversification of modern circuits rather than geometric scaling. One of the promising technologies in this field has been spintronics devices which exploits the spin of an electron instead of its charge. Furthermore, the integration of magnetic spintronics devices with MOSFET circuits - demonstrated by commercial devices such as STT-MRAM - has opened the possibility of Systems-on-Chip (SoC) integrated circuits with both types of components.

While there are many physics-based simulators which can study the detailed dynamics of magnetic materials and many CAD tools for large scale circuit design, there is a dearth of simulation tools for circuit designers working with spintronics devices. This dissertation proposes a Verilog-A behavioral hardware model of multiferroic and spin transfer torque (STT) devices which can be incorporated within traditional large scale CAD tools such as Synopsys HSPICE. Using this simulation platform, this work explores how spintronics devices can implement several More-than-Moore applications and proposes circuit and architecture-level designs to realize those applications. This dissertation considers two promising developments in magnetic spintronics devices - logic using multiferroic materials and applications using 
spin-torque nano-oscillators (STNO).

Multiferroic materials describe a class of materials which exhibit both ferroelectric and ferromagnetic behaviors. The combination of these two attributes allows for the control of the magnetic state of the material using an electric field rather than a magnetic fielda process known as electrically assisted magnetic switching (EAMS). This work develops a VerilogA model which captures the EAMS process in multiferroic materials through a compact thermodynamic model. This model demonstrates that multiferroic nanopillars can not only be used to represent binary logic bits, but they also provide a third state that can be used for reconfiguration similar to traditional field programmable gate arrays (FPGAs). This dissertation describes the operations of a reconfigurable array of magnetic automata (RAMA) based on multiferroic nanopillars which can perform ultra-low power computation.

Yet another method to control the magnetization of materials using electrical currents is through the spin transfer torque (STT) effect. The STT effect manifests in magnetic tunnel junctions (MTJ) when a DC current is applied through the junctions. The modularity of the proposed Verilog-A model can be modified to include the STT effect to simulate the behavior of spin torque nano-oscillators. Furthermore, this model shows that connecting multiple STNOs leads to complex behaviors such as synchronization. In an array of parallel-connected STNOs, this synchronization can be exploited for pattern recognition applications. Finally, this dissertation explores applications using STNOs as on-chip RF components such as bandpass and bandstop filters. The nanoscale dimensions, electrically tunable frequencies and integration with MOSFETs make STNOs an attractive option for future RF components of SoC integrated circuit. 


\section{Acknowledgments}

During my time at UVa, I have been privileged to work with some amazing people, without whom this dissertation would not have been possible. First and foremost, I would like to thank my advisor Prof. Mircea Stan. He has been one of the best mentors any graduate student can hope for. Prof. Stan has allowed me immense freedom and opportunity to pursue exciting research work, has guided me and inspired new ideas, and for always pushed me to achieve my full potential. He has taught me how to become a better engineer, and more importantly, a better thinker.

I'm also greatly indebted to the NanoSTAR group led by Prof. Jiwei Lu and Stuart Wolf. They have greatly shaped much of the research work that has become part of this dissertation. They have always made themselves available whenever I have needed help and allowed me to work freely in their lab to design experiments and test new ideas. Similarly, my dissertation would be incomplete if not for Linqiang Luo. His help with the experimental aspects of this project were invaluable in designing, understanding and validating the results of my models. My discussions with him helped me explore and understand many different aspects of my own work and he was there to support me during the difficult times of my graduate studies.

I would also like to thank my $\mathrm{PhD}$ committee members for their valuable inputs which 
have shaped my dissertation. Prof. Bobby Weikle and Art Lichtenberger provided critical help in designing and understanding the RF aspects of my research work. Similarly, Prof. Harriott had influenced my work on multiferroic materials by providing important critiques during my $\mathrm{PhD}$ exam (before joining my committee). Those critiques were very useful in developing a better architecture for RAMA device.

I'm grateful to all my great friends in the High Performance Low Power (HPLP) lab who have supported me all these years. They are the ones that have made graduate student life enjoyable and fun, and being surrounded by such talented people has kept me motivated to becoming a better student.

Finally, I would like to thank my family for their love and support. My loving wife, Farhana, has given meaning to my life and without her encouragements I would not have made it this far. To my parents, words cannot express my gratitude-I only strive to become as good of persons as you are. And to my brother who always knew how to cheer me up. Thank you all. 


\section{Contents}

Contents vi vi

List of Figures. . . . . . . . . . . . . . . . . . viii

1 Introduction $\quad \mathbf{1}$

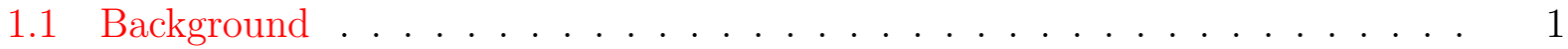

1.2 Thesis Outline . . . . . . . . . . . . . . . . . . . 5

2 Spintronic Framework $\quad 7$

2.1 Spins to Magnets . . . . . . . . . . . . . . . . . . . 7

2.2 Magnetic Ordering . . . . . . . . . . . . . . . . . . . . . 9

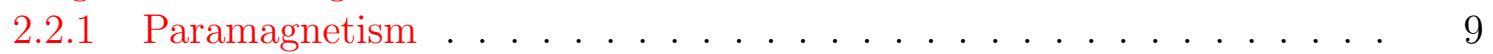

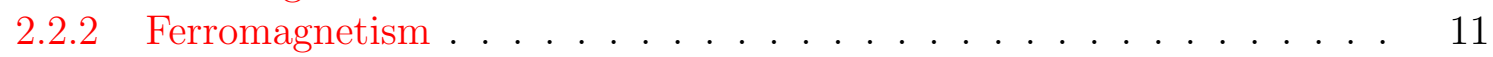

2.3 Landau-Lifshitz-Gilbert Equation . . . . . . . . . . . . . . . . . . 13

2.3.1 Ferromagnetic Dynamics . . . . . . . . . . . . . . . . . 13

2.3.2 Effective Molecular Field . . . . . . . . . . . . . . . . . 15

2.4 Verilog-A Framework . . . . . . . . . . . . . . . . . 19

2.4.1 Micromagnetics . . . . . . . . . . . . . . . . . . 19

2.4.2 Macrospin Model . . . . . . . . . . . . . . . . . 20

3 Multiferroic Spintronic Devices 22

3.1 Multiferroic Materials . . . . . . . . . . . . . . . . . . . . 23

3.1.1 Electrically Assisted Magnetic Switching . . . . . . . . . . . . . . . 24

3.1 .2 Self-assembly . . . . . . . . . . . . . . . . 26

3.2 Thermodynamic Multiferroic Model . . . . . . . . . . . . . . . 26

3.2.1 Landau-Devonshire Theory . . . . . . . . . . . . . . . . . . . . 27

3.2 .2 Coupling to Strain . . . . . . . . . . . . . . . . . . . . . . . . . . . . . . . . 30

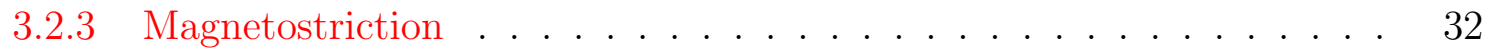

3.3 Multiferroic Verilog Model . . . . . . . . . . . . . . . . . . . 35

4 Reconfigurable Array of Magnetic Automata 38

4.1 Magnetic Quantum Cellular Automata . . . . . . . . . . . . . . . 38

4.2 RAMA: A Multiferroic MQCA . . . . . . . . . . . . . . . . 39

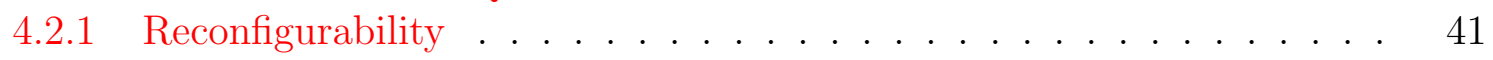

4.2 .2 Input \& Output . . . . . . . . . . . . . . . . . . . . . . . . . 42

4.2.3 Half Select Problem . . . . . . . . . . . . . . . . . . . 42 
4.3 RAMA Circuits . . . . . . . . . . . . . . . . . . 43

4.4 RAMA Architecture . . . . . . . . . . . . . . . . . 46

4.4.1 MQCA Tiling Logic . . . . . . . . . . . . . . . . . . . 46

4.4.2 Logic Primitives \& Clocking . . . . . . . . . . . . . . . . . 48

$4.4 .33 \times 3$ MQCA Tiles . . . . . . . . . . . . . . . . . . 50

4.4 .4 Single-bit Full Adder . . . . . . . . . . . . . . . . . . . . . 51

4.5 RAMA Benchmarking . . . . . . . . . . . . . . 53

5 Modeling of Spin-Torque Nano-Oscillators 55

5.1 Spin Dependent Transport . . . . . . . . . . . . . . . . . . . 56

5.2 Spin Transfer Torque . . . . . . . . . . . . . . . . . . . . . . 59

5.3 Modeling the Dynamic Behavior of STNOs . . . . . . . . . . . . . . 60

5.4 Self-oscillation in STNOs . . . . . . . . . . . . . . . . . . . . . . 62

5.4.1 Universal Oscillator Model . . . . . . . . . . . . . . . . 62

5.4 .2 Precession Generation . . . . . . . . . . . . . . . 68

5.4 .3 Model Validation . . . . . . . . . . . . . . . . . . 72

5.5 Electrically Coupled STNOs . . . . . . . . . . . . . . . . . . . 74

5.5.1 Magnetic vs. Electrical Synchronization . . . . . . . . . . . . 74

5.5.2 Synchronization Dynamics . . . . . . . . . . . . 75

5.6 STNO Geometry \& Oscillations . . . . . . . . . . . . . . . . . . 77

5.6.1 Harmonicity \& Signal Strength . . . . . . . . . . . . . . . . 79

5.6 .2 Dual Barrier MTJ . . . . . . . . . . . . . . . . 81

6 Applications of Synchronized Spin Torque Nano-Oscillators 83

6.1 STNO Array as an Oscillatory Neurocomputer . . . . . . . . . . . . . 83

6.2 Pattern Recognition . . . . . . . . . . . . . . . . . 85

6.3 Texture Segmentation . . . . . . . . . . . . . . . . 87

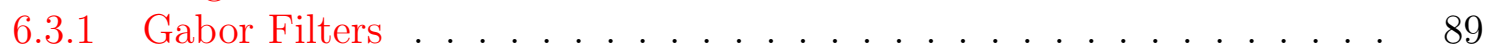

6.3.2 Oscillator-based Filtering . . . . . . . . . . . . . . 90

6.3.3 Texture Detection . . . . . . . . . . . . . . . 93

6.4 Radio Frequency Circuits . . . . . . . . . . . . . . . . . . . . 94

7 Conclusions $\quad 98$

7.1 Future Work . . . . . . . . . . . . . . . . . . . . . . . 99

$\begin{array}{ll}\text { Appendix: Simulation Parameters } & 101\end{array}$

$\begin{array}{ll}\text { Bibliography } & 102\end{array}$ 


\section{List of Figures}

1.1 Evolution of Moore's Law . . . . . . . . . . . . . . . . . . . . . 3

2.1 Magnetic ordering in crystal lattice . . . . . . . . . . . . . . . . 9

2.2 Spontaneous magnetization . . . . . . . . . . . . . . . . . . . 12

2.3 Verilog-A Model of Magnetization Dynamics . . . . . . . . . . . . . . . . 21

3.1 Electrically Assisted Magnetic Switching . . . . . . . . . . . . . . . 25

3.2 Self-assembly of Multiferroics . . . . . . . . . . . . . . . . . 26

3.3 Ferroelectric Hysteresis . . . . . . . . . . . . . . . . . . . . . . . . . . . . . . 29

3.4 Interface between Bismuth Ferrite and Cobalt Ferrite . . . . . . . . . . . 34

3.5 Multiferroic Model Validation . . . . . . . . . . . . . . . . . . 36

3.6 Switching vs. Configuration . . . . . . . . . . . . . . . . . . 37

4.1 MQCA Encoding \& Gates . . . . . . . . . . . . . . . . . . . . . . . . . . 39

4.2 Structure of RAMA Array . . . . . . . . . . . . . . . . . . . . 40

4.3 RAMA Switching . . . . . . . . . . . . . . . . . . . . 41

4.4 Half-Select Problem . . . . . . . . . . . . . . . . . . . . . . . 43

4.5 RAMA Checkerboard Pattern . . . . . . . . . . . . . . 44

4.6 RAMA Majority Gate . . . . . . . . . . . . . . . . . . . 45

4.7 MQCA Tiling . . . . . . . . . . . . . . . . . . . . . . . 47

4.8 MQCA Clocking . . . . . . . . . . . . . . . . . . . . . . . . . . . . 49

4.9 MQCA Orthogonal Tiles . . . . . . . . . . . . . . . . . . . . . 50

4.10 MQCA Fan-out \& Baseline Tiles . . . . . . . . . . . . . . . . . . . 51

4.11 RAMA Single-bit Adder . . . . . . . . . . . . . . . . . . . . . . 52

4.12 Single-bit Adder Waveform . . . . . . . . . . . . . . . . . 53

4.13 RAMA Benchmarking . . . . . . . . . . . . . . . . 54

5.1 In-plane MTJ Dynamics . . . . . . . . . . . . . . . . . . . . 57

5.2 Out-of-plane STNO . . . . . . . . . . . . . . . . . . . . . . . . . . . 64

5.3 Oscillation Power vs. Current . . . . . . . . . . . . . . . . . . . 70

5.4 Current vs. Frequency . . . . . . . . . . . . . . . . . . . . . . 71

5.5 Magnetic Field vs. Frequency . . . . . . . . . . . . . . . . . . . . 72

5.6 Magnetic Field Angle vs. Frequency . . . . . . . . . . . . . . . . . . . 73

5.7 Synchronization Dynamics . . . . . . . . . . . . . . . . . . . 75

5.8 Dynamics of Perpendicular STNO . . . . . . . . . . . . . . . . 78

5.9 Dynamics of Mixed Geometry STNO . . . . . . . . . . . . . . . . 79 
5.10 Dual Barrier Magnetic Tunnel Junction . . . . . . . . . . . . . . . . . . . . 80

5.11 DMTJ Fabrication . . . . . . . . . . . . . . . . . 81

6.1 STNO Array as an Oscillatory Neurocomputer . . . . . . . . . . . . . . . . 84

6.2 Toy Model of Pattern Recognition . . . . . . . . . . . . . . . . . . 85

6.3 Fourier Analysis of STNO Array . . . . . . . . . . . . . . . . 87

6.4 Texture Detection using Gabor Filters . . . . . . . . . . . . . . . . 88

6.5 Gabor Filter Orientation . . . . . . . . . . . . . . . . . . . . 89

6.6 Oscillatory Gabor Filter . . . . . . . . . . . . . . . . . . . . 91

6.7 STNO Gabor Filter . . . . . . . . . . . . . . . . . . . . . . 92

6.8 Gabor Filter Bandwidth . . . . . . . . . . . . . . . . . . . . . 93

6.9 RF Notch Filter using STNO . . . . . . . . . . . . . . . . . . . . 94

6.10 Coupled STNO Notch Filter . . . . . . . . . . . . . . . . . 96

6.11 Thermal Broadening in STNO Filter . . . . . . . . . . . . . . 97 


\section{Chapter 1}

\section{Introduction}

\section{$1.1 \quad$ Background}

Since Gordon Moore observed in 1965 that the number of transistors doubles approximately every two year, the scaling of devices and minimum feature sizes has been largely responsible for improvements in integrated circuits, and has facilitated the growth of the semiconductor industry. Increasingly intensive efforts have been directed to maintaining and extending Moore's law. However, the nature of scaling has already changed, causing major shifts such as the switch to parallelism rather than clock frequency as the primary driver of performance in microprocessors.

As MOSFET technologies approach sub-10nm technology nodes, there is a growing concern that scaling of devices in any form is slowing down, and there is a good chance that it will eventually become infeasible to cost-effectively manufacture devices below a certain feature size. The search for the next "switch" as a replacement to the silicon transistor in order to

facilitate continued scaling is on [1]. While several promising alternatives are being explored, 
each has significant issues that need to be addressed before it can be considered a realistic candidate to replace silicon and CMOS.

While Moore's law-driven scaling has traditionally focused on improving computation and memory capacity, electronic systems of the future will provide value by being multi-functional. Recent roadmaps for integrated circuits envision three domains for future direction of Moores law: 1) More Moore, 2) Beyond CMOS and 3) More than Moore (Figure 1.1). The More Moore domain is expected to deal with traditional silicon transistor and its scalability. The Beyond CMOS domain consists of various nanotechnologies beyond ultimately scaled CMOS (e.g. graphene, silicon nanowire, spintronics, etc.) which can potentially replace silicon and CMOS in the future. The More than Moore domain encompasses various disruptive paradigms which focus on functional diversity and heterogeneous integrated circuits. Morethan-Moore technologies envisage multiple functional units such as RF or bio-inspired circuits alongside traditional silicon on a single chip (System-on-Chip) or package (System-in-Package) to deliver solutions based on specific needs rather than general purpose computing. One promising nanotechnology which spans both Beyond CMOS and More-than-Moore domains is spintronics.

Spintronic devices exploit the spin of an electron rather than its charge to carry information. One common manifestation of electron spin is with ferromagnetic materials. While ferromagnets have featured in electronics since the late 1920s [3], the novelty of magnetic spintronic devices comes from the ability to control the materials magnetization through applied electric fields rather than magnetic fields. By removing the constraints of applying localized magnetic fields, spintronic devices can be miniaturized and operated at nanometer scales, allowing for densities comparable to silicon transistors [4]. With the revolutionary 


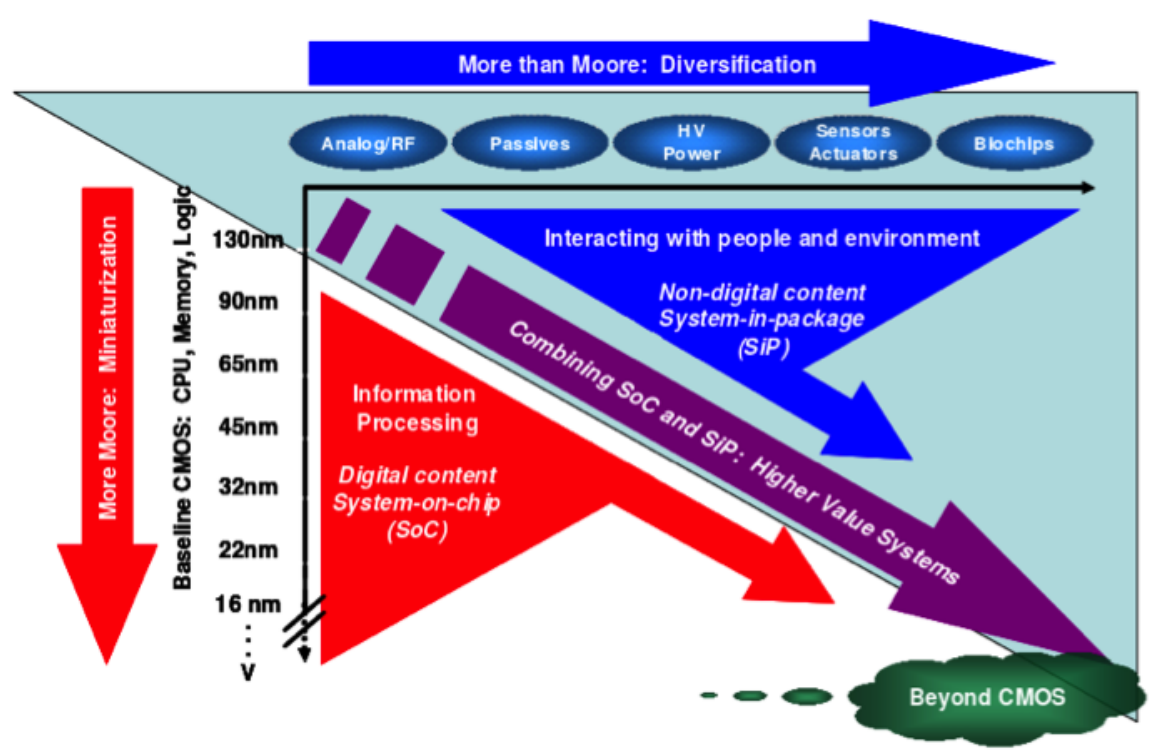

Figure 1.1: Evolution of Moore's Law. Rather than focusing on geometric miniaturization of CMOS (shown as More Moore), current evolution of technology also takes into account the functional diversification of integrated circuits (shown as More-than-Moore). Spintronic technologies in Beyond CMOS category can potentially address both scaling and functional diversities of future technologies. Image from ITRS [2].

discovery of the giant magnetoresistance (GMR) in magnetic thin films [5, 6] and the more recent developments in multiferroics materials [7], spintronic devices have become one of most promising emerging post-silicon technologies. The shift from electron charge to spin also opens up novel possibilities for logical representation - such as magnetic quantum cellular automata (MQCA) - and computation — such as oscillatory neurocomputation. Finally, the integration of magnetic spintronics devices with MOSFET circuits - demonstrated by commercial devices such as STT-MRAM has expanded the possibility for More-than-Moore type applications.

While there are several micromagnetic simulators which can study the detailed dynamics of magnetic materials and many CAD tools for large scale circuit design, there is a dearth of simulation tools for circuit designers working with spintronics devices. This dissertation proposes a Verilog-A behavioral hardware model of multiferroic and spin transfer torque (STT) 
devices which can be incorporated within traditional large scale CAD tools such as Synopsys HSPICE. Using this simulation platform, this work explores how spintronics devices can implement several More-than-Moore applications and proposes circuit and architecture-level designs to realize those applications. This proposal considers two promising developments in magnetic spintronics devices - nanomagnetic logic using multiferroic materials and applications using spin-torque nano-oscillators (STNO).

The major contributions of this dissertation are:

- The development of a spintronic simulation framework using Verilog-A and HSPICE to model the dynamics of mono-domain nanomagnets.

- Incorporation of a thermodynamic free energy model within the spintronic simulation framework to simulate the behavior of multiferroic materials.

- Demonstration of two distinct modes of operation-magnetic switching and reconfigurationwithin multiferroic nanopillars using electrically assisted magnetic switching (EAMS).

- Designing a reconfigurable array of magnetic automata (RAMA) which can perform logic operations using magnetic quantum cellular automata (MQCA) and can be reconfigured similar to traditional field programmable gate arrays (FPGAs).

- Development of a spin-transfer torque (STT) module within the spintronic simulation framework to study the behavior spin torque nano-oscillators (STNOs).

- Investigation of the effects of magnetic tunnel junction (MTJ) geometry on the mode of oscillation of STNOs. 
- Construction of an oscillatory neurocomputer using an array of electrically-coupled STNOs.

- Demonstration of complex pattern recognition, such as texture detection, by exploiting synchronization within the array of electrically-coupled STNOs.

- Designing nanoscale, tunable RF bandpass/bandstop filters using STNOs for Systemon-chip applications.

The contributions of this dissertation will provide an invaluable simulation tool to answer scientific questions about spintronic behavior of magnetic materials. Furthermore, this simulation tool will provide a platform for circuit designers to use spintronic devices alongside traditional circuit elements to explore novel architectures and applications that are possible with this emerging technology.

\subsection{Thesis Outline}

This dissertation is organized as follows. Chapter 2 will briefly explore the dynamical behavior of ferromagnets which will provide the framework for the development of spintronic device models. In Chapter 3, this framework is extended to multiferroic materials and forms the basis of the Verilog-A device model. The model is then verified with comparisons with experimental results. In Chapter 4, this multiferroic device model is used to design a Reconfigurable Array of Magnetic Automata (RAMA) which is capable of implementing logic circuits based on cellular automata computation. In addition, a novel architecture is presented which takes advantage of the multiferroic behavior to implement arbitrary reconfigurable logic. In 
Chapter 5, a Verilog-A model is developed for spin-torque devices. A major focus of this chapter deals with the rich, dynamical behavior of spin-torque nano-oscillators (STNOs). Finally in Chapter 6, the STNO Verilog-A model is used to explore several applications. The first set of applications looks at non-Boolean computation using oscillatory neural networks. The second set of applications explores systems-on-chips (SoC) using STNOs for various RF components. In Chapter 7, some future directions for the evolution of spintronic devices and their respective models are considered. 


\section{Chapter 2}

\section{Spintronic Framework}

Ferromagnets play an immense role in spintronic devices and form the basis for encoding information in magnetic devices. An understanding of the dynamics of ferromagnets under various operating conditions is crucial for the development of spintronic device models. In this chapter we will briefly explore a semiclassical theory of ferromagnets, the goal of which will be to develop the dynamical equations of motion known as the Landau-Lifshitz-Gilbert (LLG) equations. The LLG equations, while phenomenological in nature, provide accurate results for a wide range of magnetic dynamics and will form the framework the Verilog-A model of spintronic devices.

\subsection{Spins to Magnets}

Spin is an intrinsic, quantum mechanical angular momentum in elementary particles which has no classical counterpart. It was first observed in 1922 in the famous Stern-Gerlach experiments [8], where it was shown that a perpendicular magnetic field causes a splitting 
in the atomic spectra of $s$-states of hydrogen atoms. It was Wolfgang Pauli in 1927 who postulated that the electron has an additional degree of freedom which has a quantized angular momentum of $|S|= \pm \frac{\hbar}{2}$. The spin degree of freedom along with Pauli's exclusion principle of fermions was successful in explaining the organization of the periodic table and ushered in the field of quantum chemistry [9].

For charged particles, such as electrons, any angular momentum will create a magnetic moment,

$$
\vec{\mu}=g \frac{-e}{2 m_{e}} \mathbf{J}=-\gamma \mathbf{J}
$$

where $e$ is the electron charge, $m_{e}$ is the electron mass and $\mathbf{J}=\mathbf{L}+\mathbf{S}$ is the total angular momentum composed of both orbital and spin angular momenta. The Landé $g$-factor is a relativistic quantum mechanical correction and for spin- $1 / 2$ particles takes on the value $g_{s} \simeq 2$. The term $\gamma$ is known as the gyromagnetic ratio. The Bohr magneton, $\mu_{B}=-\frac{e \hbar}{2 m_{e}}$, represents the magnetic spin moment of an electron and in SI units takes a value of $-9.24 \times 10^{-24} \mathrm{~J} \cdot T^{-1}$.

Equation 2.1 suggests that for elements with unfilled valence shells (with $\mathbf{J} \neq 0$ ), there will be an overall magnetic moment per atom in a magnetic solids. While this simple argument grossly overestimates the magnetic moment in magnetic materials, it does give an intuitive picture of a magnetic crystal composed of a lattice of magnetic moments (Fig. 2.1). The orientation of these moments determines the behavior of the magnet and that will be the focus of the next section. 
(a)

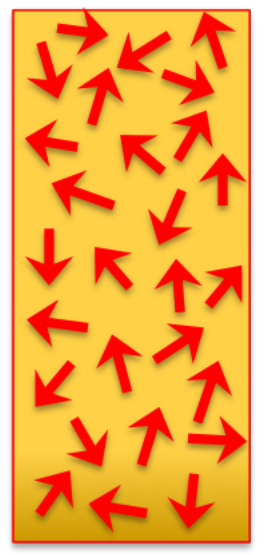

$\mathrm{H}_{\mathrm{EXT}}$

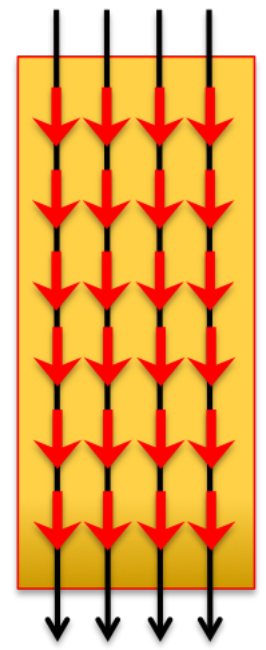

(b)

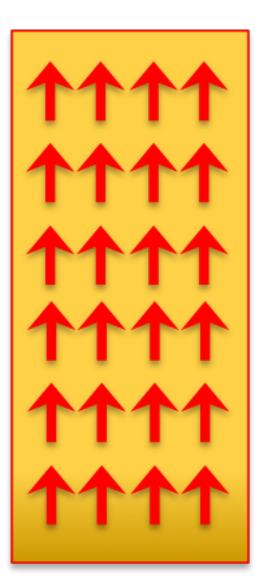

$\mathrm{H}_{\mathrm{EXT}}$

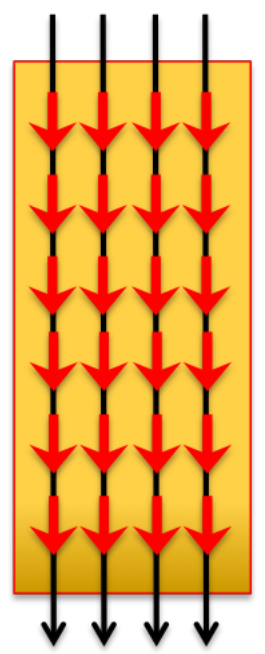

Figure 2.1: (a): Typical ordering of magnetic moments in paramagnetic materials with and without an external magnetic field. (b): Ferromagnetic materials exhibit aligned magnetization even in the absence of an external magnetic field. A nonzero applied magnetic field is needed to nullify or reverse the magnetization direction within a ferromagnet.

\subsection{Magnetic Ordering}

\subsubsection{Paramagnetism}

In this section, we explore the statistical behavior of magnetic solids and determine its equilibrium state. This will allow us to develop the dynamical nonequilibrium behavior as a perturbation from the equilibrium state. Let us consider a bulk magnetic material within a region $\Omega$ (Fig. 2.1a). Now at position $\mathbf{r} \in \Omega$, if we choose a coarse-grain unit volume $d V_{r} \ll \Omega$, such that the number of magnetic moments within the volume is large (i.e. $\gg \mu_{B}$ ), we can define a quantity known as the magnetization such that:

$$
\mathbf{M}(\mathbf{r}) \equiv \frac{1}{d V_{r}} \sum_{i} \overrightarrow{\mu_{i}}=N \mathbf{m}_{\mathbf{0}}
$$


where $N$ is the number atoms per unit volume and $\mathbf{m}_{\mathbf{0}}$ is the total magnetic moment within the unit volume.

In the absence of a magnetic field, there is no preferred orientation and there is zero average magnetization. When an external magnetic field, $\mathbf{H}_{\mathbf{e x t}}$, is applied to a magnetic moment, the energy of the system is given by:

$$
E=-\mu_{0} \mathbf{m}_{\mathbf{0}} \cdot \mathbf{H}_{\mathrm{ext}}
$$

Now if we use the Maxwell-Boltzmann distribution in which $p(E) \propto \exp \left(-\frac{E}{k T}\right)$, then the average magnetization is given by:

$$
\begin{aligned}
\langle M\rangle & =\frac{\int M p(E) d E}{\int p(E) d E} \\
& =\frac{\int_{E_{\min }}^{E_{\max }} N m_{0} \cos \theta \exp \left(\frac{\mu_{0} m_{0} H_{\text {ext }} \cos \theta}{k T}\right) d\left(-\mu_{0} m_{0} H_{\text {ext }} \cos \theta\right)}{\int_{E_{\min }}^{E_{\max }} \exp \left(\frac{\mu_{0} m_{0} H_{\text {ext }} \cos \theta}{k T}\right) d\left(-\mu_{0} m_{0} H_{\text {ext }} \cos \theta\right)} \\
& =M_{s}\left(\operatorname{coth} \beta-\frac{1}{\beta}\right)=M_{s} \mathcal{L}(\beta)
\end{aligned}
$$

where $\beta=\frac{\mu_{0} m_{0} H_{e x t}}{k T}, M_{s}=N m_{0}$ represents the case where all the magnetic moments are aligned with the external magnetic field, and $\mathcal{L}(\beta)$ is the Langevin function. For $\mu_{0} m_{0} H_{\text {ext }} \ll k T$, the Langevin function can be approximated expanded as:

$$
\mathcal{L}(\beta)=\frac{\beta}{3}+\mathcal{O}\left(\beta^{2}\right)
$$


and Eqn. 2.4 reduces to give a linear $M-H$ relationship:

$$
M=\left(\frac{\mu_{0} m_{0} M_{s}}{3 k T}\right) H_{e x t}=\chi H_{e x t}
$$

where $\chi$ is the magnetic susceptibility of the magnetic material. The vast majority of materials exhibit this linear behavior are classified as paramagnetic materials (Fig. 2.1a). Notice that in the limit of high temperature $T \rightarrow \infty$, the magnetization once again vanishes with random magnetic moments.

\subsubsection{Ferromagnetism}

However, there is a class of material, known as ferromagnetic materials, which exhibit very strong magnetization even in the absence of an external magnetic field. Materials such Fe, Co, and $\mathrm{Ni}$ and their alloys have been studied since antiquity for their properties as permanent magnets (for interesting discussion see [10]). The first accurate theory of ferromagnets was proposed by Weiss in 1907 [11], a remarkable achievement given that the quantum mechanical revolution had not yet occurred.

Weiss proposed that in ferromagnets, there was an intrinsic molecular magnetic field which would align the magnetic moments, and furthermore this field was directly proportional to the magnetization of the material:

$$
\mathbf{H}_{\mathbf{m}}=\alpha_{w} \mathbf{M}
$$

where $\alpha_{w}$ is a material dependent parameter. With this molecular field, the external magnetic 
(a)

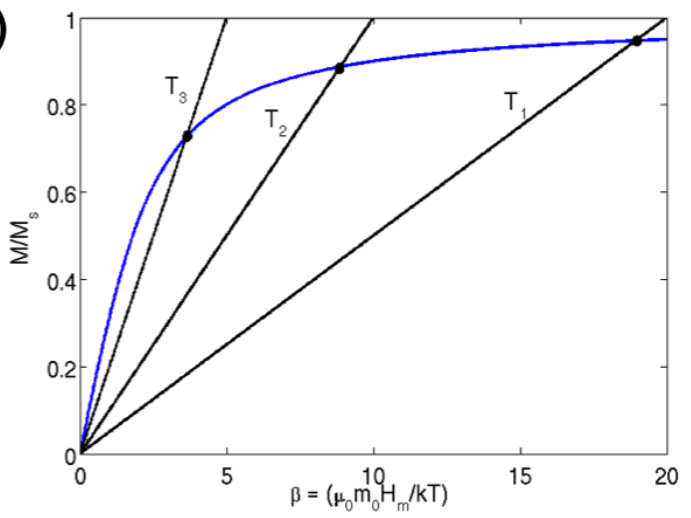

(b)

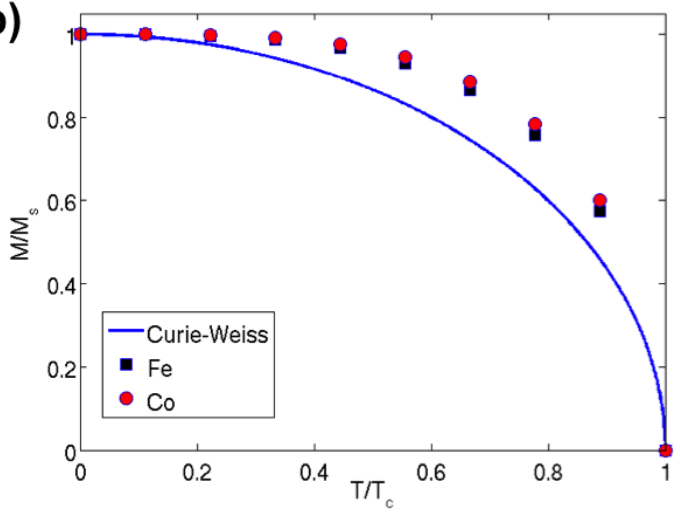

Figure 2.2: (a): Solving the Langevin relation for multiple temperatures. Here $T_{1}<T_{2}<$ $T_{3}$. As temperature increases, the spontaneous magnetization decreases until the Curie temperature is reached when the ferromagnet becomes paramagnetic. (b): Comparison of the Curie-Weiss law to experimental results [10] of Fe and Ni shows good qualitative agreement.

field in Eqn. 2.3 is be modified such that $\mathbf{H}_{\mathbf{e x t}}^{\prime}=\mathbf{H}_{\mathbf{e x t}}+\mathbf{H}_{\mathbf{m}}$. For high temperatures $(\beta \ll 1)$, we can use the approximation given by Eqn. 2.5 with the only modification being $\beta=\frac{\mu_{0} m_{0}\left(H_{e x t}+H_{m}\right)}{k T}$. This gives a $M-H$ relationship of:

$$
M=\left(\frac{T_{c} / \alpha}{T-T_{c}}\right) H_{e x t}, \chi_{F M}=\left(\frac{T_{c} / \alpha}{T-T_{c}}\right)
$$

where $T_{c}=\frac{\alpha \mu_{0} m_{0} M_{s}}{3 k}$ is the Curie temperature and Eqn. 2.8 is known as the Curie-Weiss law. This relation states that when the temperature $T>T_{c}$, a ferromagnet behaves similar to a paramagnetic material. For most metals the Curie temperature is of order $T_{c} \sim 1000 K$, and above this temperature there is only random ordering magnetic moments in the absence of a magnetic field. However, the magnetic susceptibility $\chi_{F M}$ diverges when $T=T_{c}$ indicating a phase transition to a more ordered state. For these low temperatures, the magnetization can be obtained by solving the Langevin relation 2.4 simultaneously with Eqn. 2.7 (Fig. 2.2a). This indicates that below the Curie temperature, the molecular field helps to align the 
magnetic moments to create a nonzero magnetization even without an applied external field (Fig. 2.1b).

Weiss' molecular field theory qualitatively provides a good match for the experimental data of spontaneous magnetization in ferromagnets (Fig. 2.2b). A more rigorous theory of the molecular field was provided by Heisenberg in 1931 from the point of view of quantum mechanics [10]. The discrepancies between experimental data disappear when exchange interactions between spins are taken into account.

Nonetheless, we can use thermodynamical arguments to estimate an effective molecular

field, $\mathbf{H}_{\text {eff }}$ based on the energetics of the crystalline structure and applied fields, in order to find the equilibrium state of the ferromagnet. From this equilibrium state, we can study how an arbitrary deviation from the equilibrium creates a dynamical motion in the magnetization. This motion is explored in the following section.

\subsection{Landau-Lifshitz-Gilbert Equation}

\subsubsection{Ferromagnetic Dynamics}

Assume that we have an effective molecular field $\mathbf{H}_{\text {eff }}$ which aligns the magnetic moments in equilibrium (derivation of this field will be discussed in the next section). Now let us consider a magnetic moment $\mu$ which is out of equilibrium and consider its motion in this effective molecular field. 
From classical mechanics we know that a magnetic moment $\vec{\mu}$ experiences a torque in the presence of a magnetic field:

$$
\frac{d \mathbf{J}}{d t}=\vec{\mu} \times \mathbf{H}
$$

Substituting Eqn. 2.1 yields:

$$
\frac{d \vec{\mu}}{d t}=-\gamma \vec{\mu} \times \mathbf{H}
$$

Now if we consider the dynamics within a bulk ferromagnetic material, then within a small volume $d V_{r}$, the motion of the magnetic moments can be given by:

$$
\frac{1}{d V_{r}} \frac{d \sum_{i} \overrightarrow{\mu_{i}}}{d t}=-\gamma \frac{\sum_{i} \overrightarrow{\mu_{i}}}{d V_{r}} \times \mathbf{H}
$$

which in conjunction with Eqn. 2.2 gives the relation:

$$
\frac{d \mathbf{M}}{d t}=-\gamma \mathbf{M} \times \mathbf{H}_{\mathbf{e f f}}
$$

where $\mathbf{H}_{\mathbf{e f f}}$ is the effective molecular field within the ferromagnet. The motion described by Eqn. 2.12 is the precession of the magnetization around the effective magnetic field with a Larmor frequency of $\omega=\gamma H_{\text {eff }}$. This precession would continue indefinitely if not for the dissipative forces which dampens the oscillation, aligns the magnetization with the effective magnetic field, and returns to the system to equilibrium. To model this dissipation, Gilbert proposed a phenomenological torque term [12] — akin to drag forces in classical mechanicswhose components are proportional to the velocity of the magnetization. Therefore Eqn. 2.12 
is modified as:

$$
\frac{d \mathbf{M}}{d t}=-\gamma \mathbf{M} \times \mathbf{H}_{\mathrm{eff}}+\frac{\alpha}{M_{s}} \mathbf{M} \times \frac{d \mathbf{M}}{d t}
$$

where $\alpha$ is a material dependent damping term and $M_{s}$ is the saturation magnetization. Eqn 2.13 is often recast by multiplying both sides by the magnetization and using the vector identity $\mathbf{a} \times(\mathbf{b} \times \mathbf{c})=\mathbf{b}(\mathbf{a} \cdot \mathbf{c})-\mathbf{c}(\mathbf{a} \cdot \mathbf{b})$ :

$$
\begin{aligned}
& \mathbf{M} \times \frac{d \mathbf{M}}{d t}=-\gamma \mathbf{M} \times\left(\mathbf{M} \times \mathbf{H}_{\mathrm{eff}}\right)+\mathbf{M} \times\left(\frac{\alpha}{M_{s}} \mathbf{M} \times \frac{d \mathbf{M}}{d t}\right) \\
& \mathbf{M} \times \frac{d \mathbf{M}}{d t}=-\gamma \mathbf{M} \times\left(\mathbf{M} \times \mathbf{H}_{\mathrm{eff}}\right)-\alpha M_{s} \frac{d \mathbf{M}}{d t}
\end{aligned}
$$

Now if we substitute Eqn. 2.14 into the second term of 2.13 and re-arrange the terms, we arrive at the Landau-Lifshitz-Gilbert (LLG) equation:

$$
\frac{d \mathbf{M}}{d t}=-\frac{\gamma}{1+\alpha^{2}} \mathbf{M} \times \mathbf{H}_{\mathrm{eff}}-\frac{\gamma \alpha}{\left(1+\alpha^{2}\right) M_{s}} \mathbf{M} \times\left(\mathbf{M} \times \mathbf{H}_{\mathrm{eff}}\right)
$$

The LLG equation will form the core of the Verilog-A spintronic model and can be used to describe a wide variety of magnetic phenomenon with small modifications. Now we describe a general method to derive the effective molecular field using thermodynamical arguments.

\subsubsection{Effective Molecular Field}

Weiss postulated the effective molecular field which was directly proportional to the magnetization of the material (Eqn. 2.7). While this provides a first approximation to the positive feedback mechanism driving ferromagnetism, the model can be further improved by taking 
into account the thermodynamics of the environment for the magnetic material.

In this thermodynamic model, we estimate the free energy contributions from various effects within and external to the magnetic crystal. Once we have the free energy landscape, we can derive the effective molecular field through the the functional derivative of the free energy with respect to the magnetization:

$$
\mu_{0} \mathbf{H}_{\mathrm{eff}}=-\frac{\delta G}{\delta \mathbf{M}}
$$

There are generally four main contributions to this effective field that affect most ferromagnetic materials: the externally applied magnetic field, magnetocrystalline anisotropy, exchange field, and the magnetostatic field.

\section{Zeeman field}

An applied external field, also known as the Zeeman field, has a free energy which prefers to align the magnetization in the same direction as the field. Therefore, for a bulk magnetic material a volume region $\Omega$, the free energy can be represented as:

$$
G_{Z F}=-\int_{\Omega} \mu_{0} \mathbf{M} \cdot \mathbf{H}_{\text {app }} d V
$$

\section{Magnetocrystalline Anisotropy}

In ferromagnetic bodies it is very frequent to deal with anisotropic effects, due to the structure of the lattice and to the particular symmetries that can arise in certain crystals. In fact, in most experiments one can generally observe that certain energy-favored directions exist for a 
given material, i.e. certain ferromagnetic materials, in absence of external field, tend to be magnetized along precise directions, which in literature are referred to as the easy directions. The fact that there is a "force" which tends to align magnetization along easy directions can be taken into account by means of an additional phenomenological term in the free energy functional.

The most common anisotropy effect is connected to the existence of one only easy direction, and in literature it is referred to as uniaxial anisotropy. For the simplicity, we take the easy direction to coincide with the Cartesian z-axis. Now we can define a free energy which is constant on the $x-y$ plane slices perpendicular to the $z$-axis. This free energy would have the form:

$$
G_{A N}=\int_{\Omega} K_{1}\left[1-\left(\frac{\mathbf{M} \cdot \hat{\mathbf{z}}}{M_{s}}\right)^{2}\right] d V
$$

where $K_{1}$ is the anisotropy energy density (in $\mathrm{J} / \mathrm{m}^{3}$ ) and $M_{s}$ is the saturation magnetization. For $K_{1}>0$, the minimum energy occurs when the magnetization is aligned with $z$-axis which represents the easy axis. Meanwhile for $K_{1}<0$, the minimum energy when the magnetization is perpendicular to the $z$-axis and it represents the easy plane anisotropy.

\section{Exchange Field}

The exchange field results from spin-spin interaction between neighboring lattice sites. The full derivation of spin exchange requires a quantum mechanical treatment (See Appendix A), but it can be understood as the tendency of neighboring magnetic moments to align in parallel with each other (anti-parallel for antiferromagnetic materials). In this respect, an energy term which penalizes magnetization disuniformities is introduced in the free energy. 
The free energy can expressed as:

$$
G_{E X C}=\int_{\Omega} A\left[\nabla \cdot\left(\frac{\mathbf{M}}{M_{s}}\right)\right]^{2} d V
$$

where $A$ is the exchange constant determines the strength of the correlation between neighboring magnetic moments and is typically on the order of $\sim 10^{-11} \mathrm{~J} / \mathrm{m}$.

\section{Magnetostatic Field}

Magnetostatic interactions represent the way the elementary magnetic moments interact over long distances within the body. In fact, the magnetostatic field at a given location within the body depends on the contributions from the whole magnetization vector field. Magnetostatic interactions can be taken into account by introducing the appropriate magnetostatic field $\mathbf{H}_{\mathbf{m}}$ according to Maxwell equations for magnetized media:

$$
\begin{cases}\nabla \cdot \mathbf{H}_{\mathbf{m}}=-\nabla \cdot \mathbf{M} & \text { in } \Omega \\ \nabla \cdot \mathbf{H}_{\mathbf{m}}=0 & \text { in } \Omega^{C} \\ \nabla \times \mathbf{H}_{\mathbf{m}}=\mathbf{0} & \end{cases}
$$

The energy stored within a magnetic field is given by:

$$
U_{m}=\int_{\Omega_{\infty}} \frac{1}{2} \mu_{0} \mathbf{H}_{\mathbf{m}}^{\mathbf{2}} d V
$$


Now if we express $\mathbf{H}_{\mathbf{m}}=\mathbf{B} / \mu_{0}-\mathbf{M}$ and realize that $\mathbf{B}$ and $\mathbf{H}_{\mathbf{m}}$ are orthogonal, then the free energy becomes:

$$
G_{M S}=-\int_{\Omega} \frac{1}{2} \mu_{0} \mathbf{M} \cdot \mathbf{H}_{\mathbf{m}} d V
$$

We observe that magnetostatic energy expresses a nonlocal interaction, since the magnetostatic field functionally depends on the whole magnetization vector field.

\subsection{Verilog-A Framework}

\subsubsection{Micromagnetics}

In order to simulate the behavior of spintronic devices, it is important to have an accurate model of ferromagnets. Micromagnetic simulators, such as OOMMF [13] and $\mu$ Mag [14], are numerical solvers which describe the equilibrium configuration of the magnetization in a ferromagnet, or the dynamical response to an applied magnetic field or spin transfer torque. Micromagnetics is a phenomenological description of magnetism which is designed to model spatially non-uniform distribution of magnetization within a ferromagnet.

Micromagnetic simulators use various numerical methods such as finite difference (OOMMF) or finite element $(\mu \mathrm{Mag})$ to spatially divide a magnetic media into small volume cells. Within these cells, the simulator calculates the energy landscape and apply method of steepest descent to find the effective molecular field as in Eqn 2.16. Then the simulator uses the LLG equation (Eqn. 2.15) to update the local magnetization of the cell.

While micromagnetic simulators provide accurate dynamics of ferromagnets, they also tend to be computationally intensive. Furthermore, from the perspective of a circuit designer, 
micromagnetic simulators do not provide a way to interface with other electronic components which are necessary for complete designs. Therefore, a circuit designer requires a hardwarebased spintronic device model, similar to transistor models, which can be integrated into VLSI CAD tools. This work proposes a behavioral Verilog-A model which uses a compact version of the LLG equation (Eqn. 2.15) to simulate the behavior of ferromagnets.

\subsubsection{Macrospin Model}

In order to construct a spintronic device model, couple of challenges needed to be addressed. First, the model needed to capture the dynamic behavior described by micromagnetic simulators within a hardware language framework. Second, since circuit simulators work by solving Kirchhoffs laws for electrical circuits, any spintronic device model would have to bridge the gap between its magnetic properties and the need for electrical signals which circuit simulators understand.

In order to merge the two domains, behavioral Verilog-A hardware language was used to describe the LLG equations of motion, while Synopsys HSPICE circuit simulator was used to integrate the model with the traditional circuit design framework. The Verilog-A model began by making a macrospin approximation to the LLG equations of motion. The macrospin approximation assumes that the magnetization of a sample stays spatially uniform throughout its motion and can be treated as a single macroscopic spin. This eliminates the complex calculations for the exchange (Eqn. 2.19) and magnetostatic energies (Eqn. 2.22). Since the spatial variation of the magnetization is frozen out, exploring the dynamics of magnetic systems is much more tractable using the macrospin approximation than it is using 

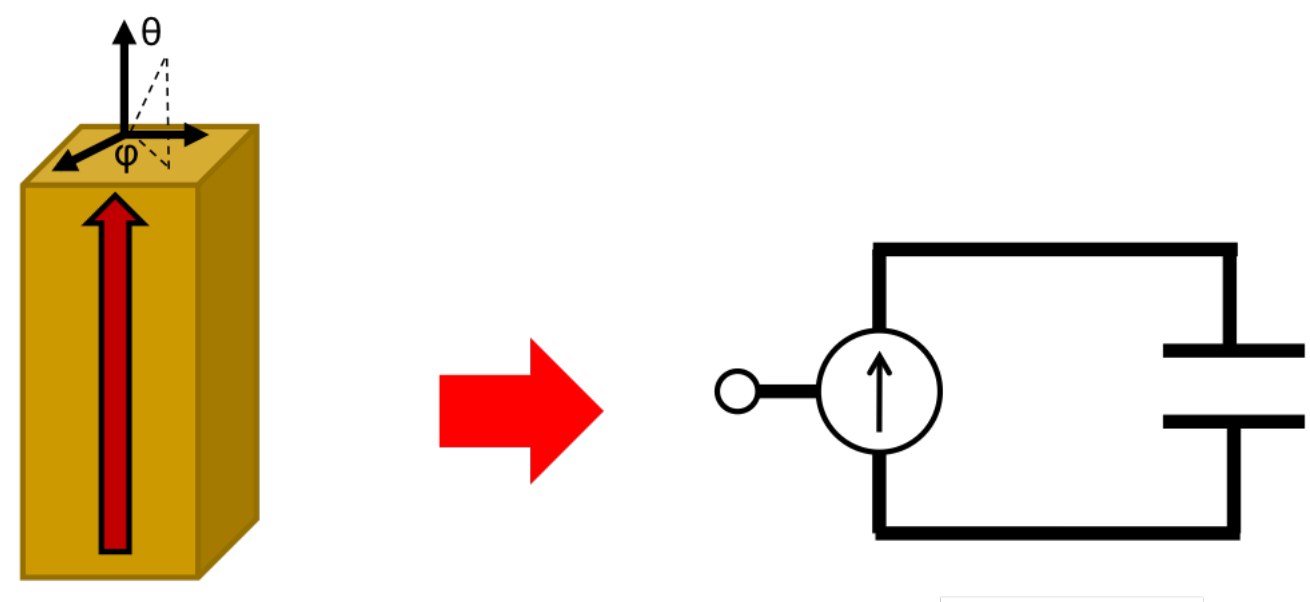

$$
\frac{d \theta}{d \tau}=[\cdots]
$$

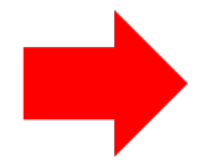

$$
\frac{d V}{d t}=\frac{I}{C}
$$

Figure 2.3: In the macrospin approximation, the magnetic body is treated as having only a single magnetic moment. The orientation of this moment can be represented as spherical angles whose equations of motion can be mapped to the voltage of a capacitor in an integration circuit.

full micromagnetic simulations.

More importantly, the macrospin approximation allows the LLG equations to be decomposed into two sets of differential equations representing the spherical angles of the magnetization. Solving these differential equations gives the dynamic magnetization behavior of the ferromagnet. Interestingly, solving these differential equations is equivalent to solving for the voltage of a charging capacitor connected with the appropriate time varying current source (Fig. 2.3) [15, 16]. This analogous behavior allows the LLG equations to be represented as a circuit which HSPICE can solve. In the following chapters we will use this framework to capture the equations of motion for two types of spintronic devices - multiferroic devices and spin torque nano-oscillators. 


\section{Chapter 3}

\section{Multiferroic Spintronic Devices}

In the previous chapter, we developed the framework to model spintronic devices. In this chapter, we will modify that framework to incorporate the behavior of multiferroic materials. First, we begin with a brief background in multiferroic phenomenon from which we develop a thermodynamic theory of multiferroics. We include this theory within the Verilog-A spintronic framework to produce a simulation framework for multiferroic devices. We then use this device model to describe a novel device consisting of an ordered array of multiferroic nanopillars which is capable of encoding information through its magnetization. Furthermore, we show that this array is capable of cellular automata type computation and can be used to implement a reconfigurable logic device similar to field programmable gate arrays (FPGAs). Finally, we discuss an architecture for this reconfigurable array of magnetic automata (RAMA) which is capable of implementing large scale arbitrary circuits. 


\subsection{Multiferroic Materials}

Multiferroic materials with coexistence of at least two ferroic orders (ferroelectric, ferromagnetic, or ferroelastic) have drawn increasing interest due to their potential for applications as multifunctional devices. In multiferroic materials, the coupling interaction between the different order parameters could produce new effects, such as magnetoelectric (ME) effect.The magnetoelectric response is the appearance of an electric polarization $\mathbf{P}$ upon applying a magnetic field $\mathbf{H}$ (i.e., the direct $\mathrm{ME}$ effect, designated as $\mathrm{ME}_{\mathrm{H}}$ effect: $\mathbf{P}=\alpha \mathbf{H}$ ) and/or the applying an electric field $\mathbf{E}$ (i.e., the converse $\mathrm{ME}$ effect, or $\mathrm{ME}_{\mathrm{E}}: \mathbf{M}=\alpha \mathbf{E}$ ).

Magnetoelectricity has been observed as an intrinsic effect in some natural material systems at low temperature, which have been under intensive study recently [17, 18, 19], motivated by potential applications in information storage, spintronics, and multiple-state memories. Although several compound families have been widely investigated as multiferroic ME materials, a high inherent coupling between multiferroic order parameters (especially above room temperature) has not yet been found in a single-phase compound, which hinders their applications. Research progress in single-phase multiferroic ME materials have been summarized in recent review articles [20, 21].

Alternatively and with greater design flexibility, multiferroic ME composites made by combining piezoelectric and magnetic substances together have drawn significant interest in recent years due to their multifunctionality, in which the coupling interaction between piezoelectric and magnetic substances could produce a large ME response [22] (e.g., several orders of magnitude higher than that in those single phase ME materials so far available) at room temperature. 
The ME effect in composite materials is known as a product tensor property [23], which results from the cross interaction between different orderings of the two phases in the composite. Neither the piezoelectric nor magnetic phase has the ME effect, but composites of these two phases have remarkable ME effect. Thus the ME effect is a result of the product of the magnetostrictive effect (magnetic/mechanical effect) in the magnetic phase and the piezoelectric effect (mechanical/electrical effect) in the piezoelectric one, namely [22]:

$$
\mathrm{ME}_{\mathrm{E}}=\left(\frac{\text { electrical }}{\text { mechanical }}\right) \times\left(\frac{\text { mechanical }}{\text { magnetic }}\right)
$$

This is a coupled electrical and magnetic phenomenon via elastic interaction. That is, for the $\mathrm{ME}_{\mathrm{E}}$ effect, when a electric field is applied to a composite, the polarization phase changes its shape piezoelectrically. The strain is then passed along to the magnetostriction phase, resulting in a magnetization change. Thus, the ME effect in composites is extrinsic, depending on the composite microstructure and coupling interaction across magnetic-piezoelectric interfaces. In this work, we model the composite, multiferroic phase created by ferromagnetic cobalt ferrite $\left(\mathrm{CoFe}_{2} \mathrm{O}_{4} ; \mathrm{CFO}\right)$ embedded in ferroelectric bismuth ferrite $\left(\mathrm{BiFeO}_{3} ; \mathrm{BFO}\right)$.

\subsubsection{Electrically Assisted Magnetic Switching}

The use of electrical field to effect the magnetic properties of a ferromagnet was first demonstrated by Zavaliche et. al [24, 25, 26] in a composite CFO-BFO multiferroic structure. In that work, it was proposed that in a CFO-BFO matrix, an applied electric field can induce a magnetic switching using a magnetic field much weaker than the coercivity of the ferromagnet — an effect known as electrically assisted magnetic switching (EAMS). 
(a)

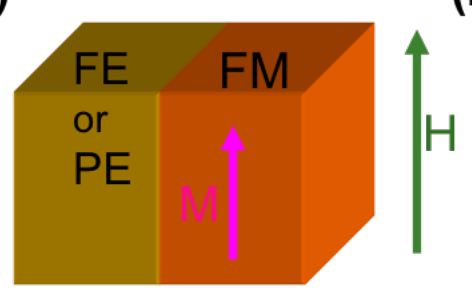

(b)

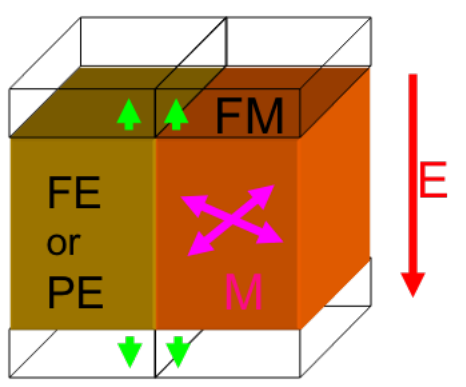

(c)

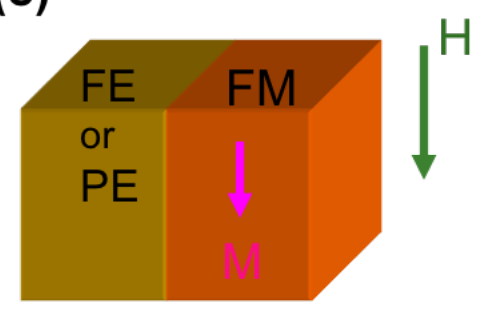

Figure 3.1: (a): Initial state of nanopillars. (b): An applied electric field induces a stress causing the magnetization of the pillar to switch in-plane. (c): Writing can be done using a small biasing magnetic field and removing the polarization with a reverse electric field.

This switching process is shown in Figure 3.1. An initial state with perpendicular magnetic anisotropy and up magnetization is sketched in Figure 3.1a. As the electrical field is ramped up, the $\mathrm{BiFeO}_{3}$ matrix goes through a phase transition during switching (Fig. 3.1b) and the compressive stress reaches a maximum. Because of the intimate contact between the two ferroic components, this compressive stress is transferred to the highly magnetostrictive $\mathrm{CoFe}_{2} \mathrm{O}_{4}$ nanopillars $\left(\lambda_{100}=-350 \times 10^{6}\right)$. Consequently, the nanopillars exhibit an increased perpendicular magnetic anisotropy. Given the negative nanopillars magnetostriction, one would expect that most of them will undergo a transient change into the perpendicular magnetic direction. A fourfold in-plane magnetic anisotropy takes over, and a spin reorientation transition from out-of-plane to in-plane may occur (Fig. 3.1b) to minimize the anisotropy energy. As the piezostress is relieved following the removal of the applied electric field, the initial out-of-plane magnetic anisotropy takes over and magnetization may flip either up or down with equal probabilities (Fig. 3.1c). At this moment, a very small out-of-plane magnetic can switch the nanopillar into a preferred direction to perform a "write" operation. 
(a)

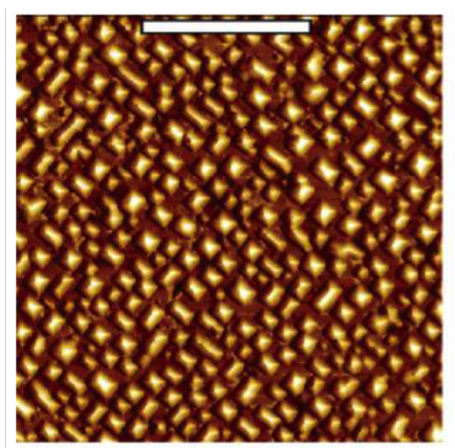

(b)

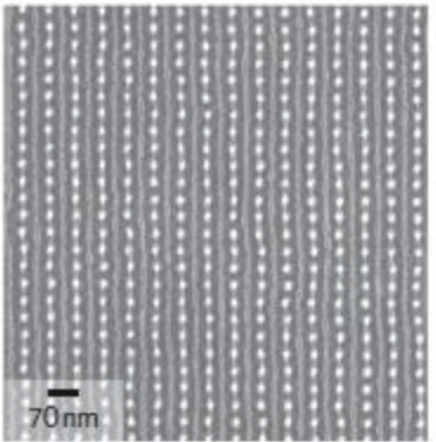

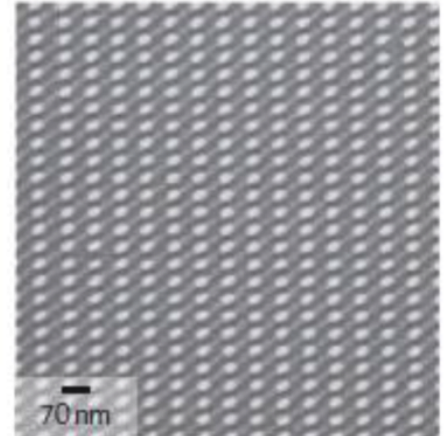

Figure 3.2: (a) AFM image of self-assembled CFO pillars in a BFO matrix [24]. (b) SEM images of block copolymer templates [28].

\subsubsection{Self-assembly}

One of the most promising aspects of multiferroic materials is the possibility of self-assembling nanostructures. It has been shown experimentally that the CFO nanostructures can be self-assembled into columnar nanopillar structures within a BFO matrix (Fig. 3.4) [24]. One of the challenges that remain is to pattern these pillars into a regular set of arrays. Several methods have proposed to address this issue, the most promising of which is block copolymerization $[27,28]$. This approach uses alternating chains of molecules on a substrate to seed the growth of the selfassembled nanostructures (Figure 3.4). Using these bottom-up techniques could allow for the creation of smaller nanostructures that cannot be created using conventional approaches. Furthermore, self-assembled structures can provide a more economical alternative to lithographic processing.

\subsection{Thermodynamic Multiferroic Model}

The theory of multiferroic materials can be developed through energy considerations similar to the dynamics of a ferromagnet (Eqn. 2.15). This section briefly describes the theory developed 
by Landau and Devonshire for ferroelectric materials [29] to model the polarization and strain response within the $\mathrm{BFO}$ material. Then this strain response is linked to ferromagnetic $\mathrm{CFO}$ via the magnetostriction effect. The magnetostriction adds an additional term to the free energy (Eqn. 2.16) which modifies the effective molecular field. This thermodynamic model of multiferroic can easily be implemented through the Verilog-A framework and provides important insights into multiferroic switching.

\subsubsection{Landau-Devonshire Theory}

A fundamental postulate of thermodynamics applied to a ferroelectric is that its free energy F can be generally expressed as a function of ten variables (three components of polarization, six components of the stress tensor, and finally one of temperature). Our goal here is to write down an ansatz for this free energy, using symmetry arguments whenever possible to pare down the number of terms [30]. The second important thermodynamic principle that we will also employ is that the values of the dependent variables in thermal equilibrium are obtained at the free energy minimum when the free energy is optimized.

We make the key approximation that in the vicinity of a phase transition we can expand the free energy in powers of the dependent variables with coefficients that can be fit to experiment or gleaned from crystal symmetry arguments [31]. In the best case, we may be able to truncate this series with only a few terms. In order to be more specific, let us take a simple example where we expand the free energy in terms of a single component of the polarization; for the moment we ignore the strain field, which we will revisit in the next section. We shall choose the origin of energy for the free unpolarized, unstrained crystal to 
be zero, and hence we can write:

$$
F(P, T)=\frac{1}{2} a_{0}\left(T-T_{0}\right) P^{2}+\frac{1}{4} b P^{4}+\frac{1}{6} c P^{6}-E P
$$

where we have truncated the power series at the sixth term, and $a_{0}, b$ and $c$ are coef- ficients that are related to the material properties. Just as in ferromagnets, the first term is related to the Curie temperature $\left(T \sim T_{0}\right)$ where the polarizations become ordered. Therefore, for ferroelectric materials the first term in Eqn. 3.2 is always negative. Furthermore, the other coefficients $b$ and $c$ are assumed to be temperature independent. Since the energy should increase as $P \rightarrow \infty$, the sign of $c$ should also be positive as the leading term.

The equilibrium configuration is determined by finding the minima of free energy, i.e. $\frac{\partial F}{\partial P}=0$. This equation gives us an expression for the electric field $\mathrm{E}$ as a function of the polarization:

$$
E=a_{0}\left(T-T_{0}\right) P+b P^{3}+c P^{5}
$$

Thus for most materials where $b=c=0$, there is a linear relationship between electric field and polarization. However for nonzero coefficients, we notice that for each value of the electric field, there can be multiple equilibrium points. These materials exhibit the nonlinear hysteretic behavior which characterize most ferroelectrics (Fig. 3.3).

When $b>0$, a continuous transition occurs at $T=T_{0}$, and the free energy will evolve continuously as a function of decreasing temperature that has a minima at finite polarizations $P= \pm P_{0}$. This spontaneous polarization can be estimated by setting $E=0$ in Eqn. 3.3; since all the coefficients are positive, we will only retain the two lowest-order terms. The 


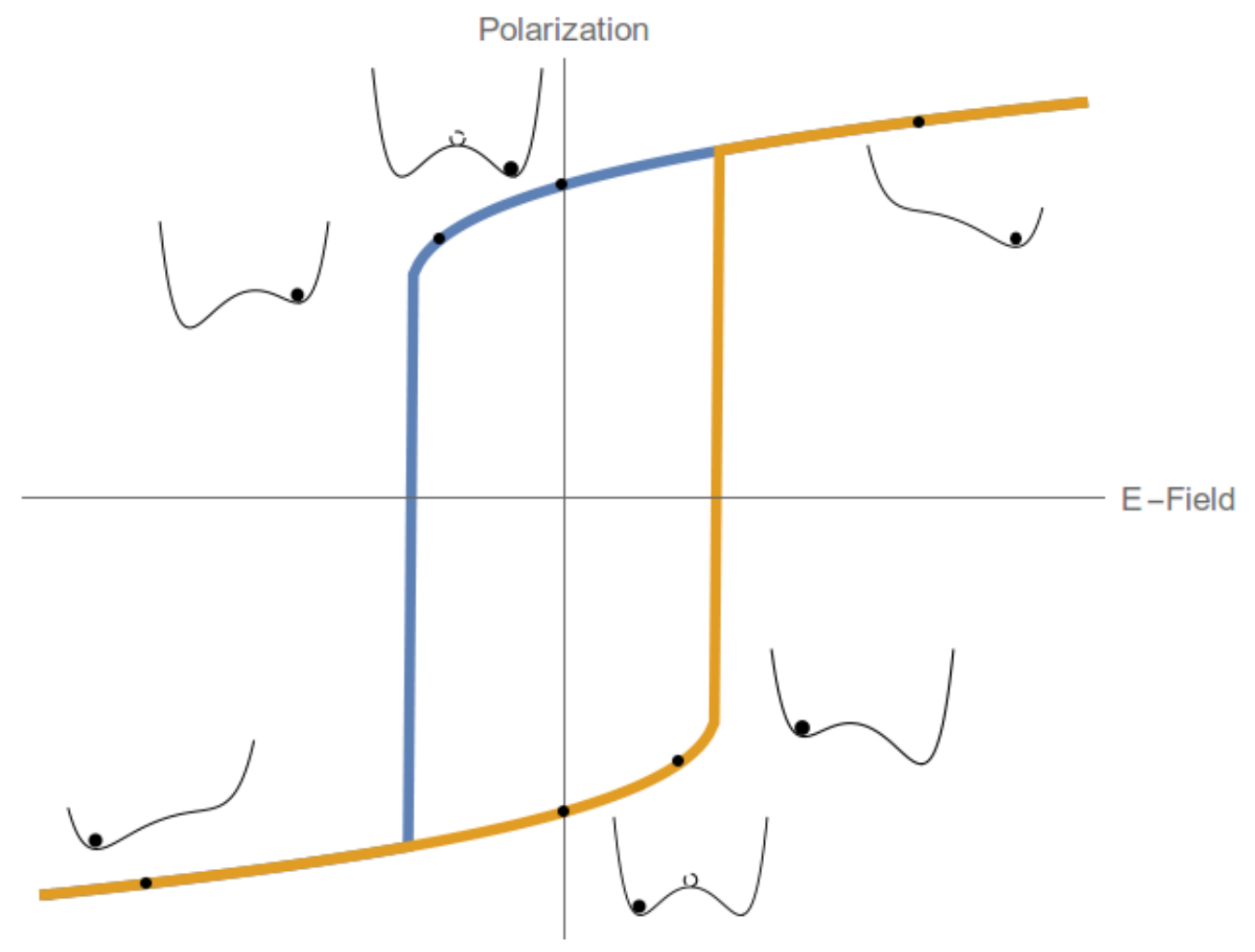

Figure 3.3: Hysteresis loop of ferroelectric material showing nonlinear behavior of the polarization in response to an electric field. The insets show the energy vs. polarization (z-direction) at different points on the hysteresis loop.

result is that the spontaneous polarization $P_{s}$ will increase with decreasing temperature from the Curie temperature $T_{0}$.

In the case of $E=0$, the free energy landscape is symmetric forming a metastable state at zero polarization (see insets in Fig. 3.3). However, a slight deviation from this state forces the system to "choose" one of the spontaneous polarization points. When an electric field is applied, it tilts the energy landscape creating a single global minima. When the energy barriers are sufficiently lowered by the electric field, the polarization rapidly switches to the other equilibrium state thus displaying the hysteretic behavior. 


\subsubsection{Coupling to Strain}

An important feature of ferroelectric materials is their great sensitivity to elastic stress. In order to understand why this is so, we can again take recourse to Landau theory by adding in strain dependent terms to Eqn. 3.2. The strain in a solid is measured by how the displacement $\vec{u}$ of a point in the solid varies with position $\vec{r}$, and since this is the dependence of a vector upon a vector, the answer is a tensor: the strain is usually defined as:

$$
\epsilon_{i j}=\frac{1}{2}\left(\frac{\partial u_{i}}{\partial r_{j}}+\frac{\partial u_{j}}{\partial r_{i}}\right)
$$

where $i, j$ indicate the $x, y, z$ components of the vectors. The strain is therefore a second degree symmetric tensor with six independent components.

In general, the polarization will couple to one or more types of strain, and specifically which ones can generally be seen by inspection. Consider for simplicity a cubic crystal that undergoes a ferroelectric phase transition to a state where the polarization can point along one of the six orthogonal cubic directions. Now it is clear that there is a special axis (one of the six directions after the symmetry has been broken) and so it would no longer be expected that the crystal as a whole will remain cubic one expects a distortion into a tetragonal crystal, which can be described by a tetragonal strain $\epsilon$. The fact that the lowest order coupling allowed in this case is of the form $\epsilon P^{2}$ (and not, for example, $\epsilon P$ or $\epsilon^{2} P$ ) is of course a matter of symmetry.

For a uniaxial ferroelectric, the leading order terms will be of the following form:

$$
F(\epsilon, P)=\frac{1}{2} K \epsilon^{2}+Q \epsilon P^{2}+\ldots-\epsilon \sigma
$$


Here $\epsilon$ a component of the strain field, and the first term represents Hookes law, namely that the elastic energy stored in a solid is quadratically dependent on the distortion, so $K$ is one of the elastic constants. The second term is a coupling between the elastic strain and the polarization; the fact that this is linear in the strain and quadratic in the polarization depends on the special symmetry of the transition [22]. Finally, $\sigma$ is an applied external stress on the crystal which expresses the force per area that neighboring particles of a continuous material exert on each other. Materials with a linear relation between stress and polarization are called piezoelectric.

Using the free energy, which now consists of the terms in Eqn. 3.2 and Eqn. 3.5, we can now determine the properties in equilibrium by minimizing with respect to both $P$ and $\epsilon$ :

$$
\frac{\partial F(P, \epsilon)}{\partial P}=\frac{\partial F(P, \epsilon)}{\partial \epsilon}=0
$$

If we take the second of these equations first:

$$
\frac{\partial F}{\partial \epsilon}=K \epsilon+Q P^{2}-\sigma
$$

We can examine a few different limits to better understand the results of Eqn. 3.7. Firstly, note that if the polarization is zero, we get Hookes law $\epsilon=\sigma / K$. The second - apparently trivial case - is when a stress is applied to force the strain to be exactly zero at all times. This is can occur often when crystals can be considered to be clamped by their surroundings so that no strain is allowed at all. One common situation is of a thin film which is forced to have the lattice constants matched to the substrate, and is free to relax only in the perpendicular direction. In the case of perfect clamping $\epsilon=0$, and the free energy is just as before. 
The third case to consider is when no external stresses are applied $(\sigma=0)$, and we then have

$$
\epsilon=-\frac{Q P^{2}}{K}
$$

so that a spontaneous strain occurs proportional to the square of the polarization. Notice now that we can substitute Eqn. 3.8 into Eqn. 3.5 to represent the strain free energy in terms of polarization only. Finally substituting this into Eqn. 3.2 yields:

$$
F(P, \epsilon(P))=\frac{1}{2} a_{0}\left(T-T_{0}\right) P^{2}+\frac{1}{4}\left(b-2 Q^{2} / K\right) P^{4}+\frac{1}{6} c P^{6}-E P
$$

Now with this equations we can accurately model the polarization and piezoelectric response of the ferroelectric material to an external electric field. Armed with the ferroelectric response, we can now look at the ferromagnetic response to the strain.

\subsubsection{Magnetostriction}

When a substance is exposed to a magnetic field, its dimensions change. This effect is called magnetostriction. It was discovered as long ago as 1842 by Joule, who showed that an iron rod increased in length when it was magnetized lengthwise by a weak field. The fractional change in length $\delta l / l$ is simply a strain, and, to distinguish it from the strain $\epsilon$ caused by an applied stress, we give the magnetically induced strain a special symbol:

$$
\lambda=\frac{\Delta l}{l}
$$

Magnetostriction occurs in all pure substances. However, even in strongly magnetic 
substances, the effect is usually small, usually on the order of $10^{-5}$. Although the direct magnetostrictive effect is small, and not usually important in itself, there exists an inverse effect which causes such properties as permeability and the size and shape of the hysteresis loop to be strongly dependent on stress in many materials. Magnetostriction therefore has many practical consequences, and a great deal of research has accordingly been devoted to it $[32,33,34]$.

Experimental results show that there is a close connection between the magnetostriction $\lambda$ of a material and its magnetic behavior under stress. As a result, the effect of stress on magnetization is sometimes called the inverse magnetostrictive effect. If we impose an additional strain by applying a stress, we expect that the direction of the magnetization will change. We therefore need a general relation between the direction of $M_{s}$ within a domain and the direction and magnitude of $\sigma$. But we know that, in the absence of stress, the direction of $M_{s}$ is controlled by crystal anisotropy, as characterized by the first anisotropy constant $K_{1}$. Therefore, when a stress is acting, the direction of $M_{s}$ is controlled by both $\sigma$ and $K_{1}$. When the magnetostriction is isotropic (as we assume in our system), it can be shown that the magnetostriction energy is given by:

$$
E_{m e}=-\frac{3}{2} \lambda_{100} \sigma \sin ^{2}(\theta)
$$

where $\lambda_{100}$ is a material dependent parameter measuring the strength of the magnetostriction and $\theta$ is the angle difference between $M_{s}$ and the applied stress $\sigma$. Therefore, for a negative value of $\lambda_{100}$ (as in $\mathrm{CFO}$ ), the system prefers the stress to aligned perpendicular to the saturation magnetization. 


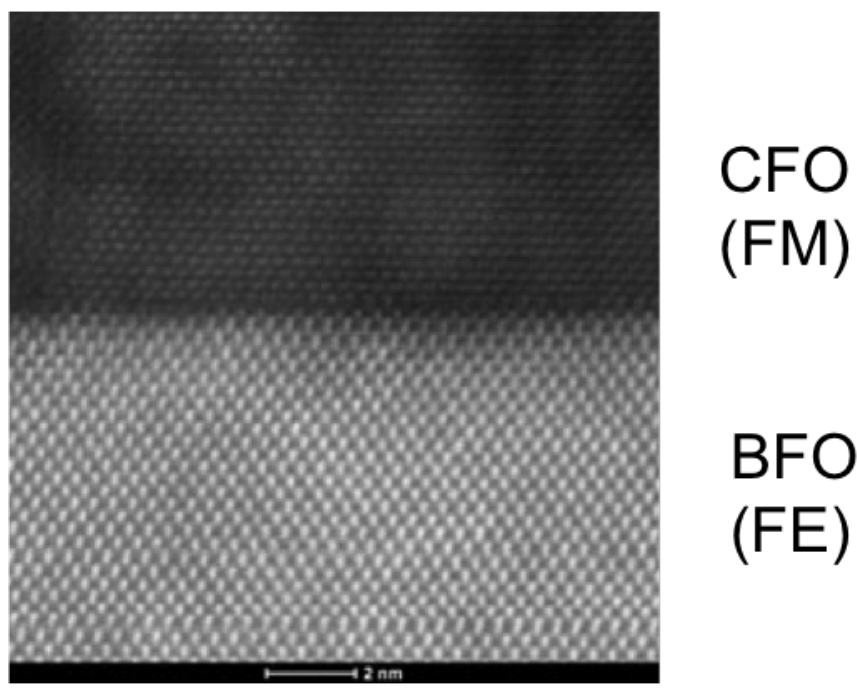

Figure 3.4: AFM image showing the interface between the ferromagnetic cobalt ferrite (CFO) and ferroelectric bismuth ferrite (BFO). Due to the intimate contact between the two layers, stress is transferred from one layer to the other.

Now in the multiferroic model, the stress experienced on the ferromagnet comes from the response of the ferroelectric to an applied electric field. Because of the intimate contact between the ferroelectric BFO and the ferromagnetic CFO (Fig. 3.4), the strain from Eqn. 3.8 is transferred as an applied stress $\left(\sigma=\epsilon Y_{C F O}\right.$, where $Y_{C F O}$ is the Young's modulus) to the CFO. With this energy specification, we can derive another term to the effective molecular field by using Eqn. 2.16 and arrived at an expression for the effective molecular field which is a function of an applied electric field. This effective molecular field will determine the transient magnetization behavior through the LLG equation (Eqn. 2.15). Having established the equations of motion for the Verilog-A model, we now validate the model with experimental results and encounter some interesting results. 


\subsection{Multiferroic Verilog Model}

The differential equations describing the evolution of the multiferroic system can then be translated into a Verilog circuit model. This is done by mapping the variable of interest (i.e. polarization, magnetization angles, etc.) to a voltage buildup on a capacitor. Then the differential equation governing the dynamics of the variable is mapped to a voltage-controlled current source which charges the capacitor. By allowing the circuit simulator to solve the equivalent circuits, we solve self-consistently the differential equations for the polarization and magnetization dynamics.

In order to verify our model, we compared the results from our model with those from experiments. In Fig. 3.5 (top left), we show the simulated polarization and strain behavior of the BFO matrix when an electric field is applied. We then compare it to the experimental data for BFO shown on the top right of Fig. 3.5. Our model does a good job capturing the polarization hysteresis curve with relatively accurate values for the remanence (simulation: $45 \mu \mathrm{C} / \mathrm{cm}^{3}$; experiment: $48 \mu \mathrm{C} / \mathrm{cm}^{3}$ ) and coercivities (simulation: $22 \mathrm{MV} / \mathrm{m}$; experiment: $20 \mathrm{MV} / \mathrm{m}$ ). However, when we compare the hysteresis in the strain (Fig. 3.5 (bottom left)), we find larger discrepancies between the model and experiment - this is because our model only takes into account stress in the out-of-plane direction, whereas in the experiment there are stresses in all directions. In addition, these stresses tend to be inhomogeneous throughout the material and depend on local crystal structures and polarization - an aspect which cannot be captured in a macrospin model. Nevertheless, our goal is not absolute accuracy, but rather the possibility of simulating large arrays as well as hybrid CMOS/nano circuits, and the model provides a good approximation to capture the important features of the multiferroic 

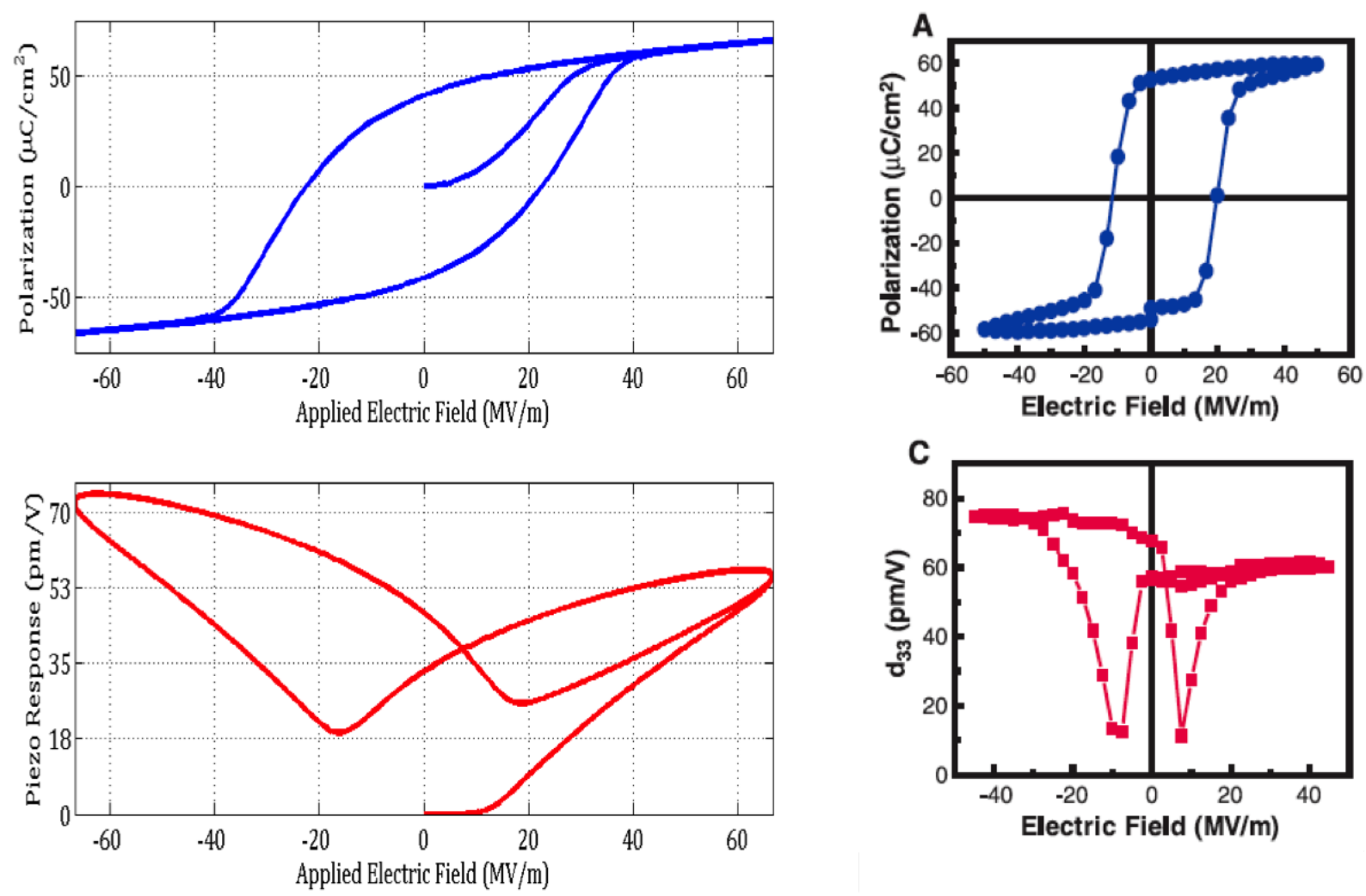

Figure 3.5: The comparison between the Verilog-A simulation of BFO and experiment [35] in ferroelectric behavior (top) and piezoelectric response (bottom).

material for such simulations.

Using our model we also examine the effect an electric field on the magnetization dynamics of the CFO nanopillars. We find that there are three regimes of operation depending on the strength of the electric field. When the applied electric field creates a stress energy that is weak compared to the crystalline and shape anisotropy energies, we find that the magnetization deviates only slightly into the in-plane direction but maintains a strong outof-plane component. However, when the stress energy is comparable to the magnetostatic energies, we see that the magnetization switches from the out-of-plane into the in-plane direction (Fig. 3.6(a)) as predicted by experiments [24, 35, 36]. The energy landscape (shown 


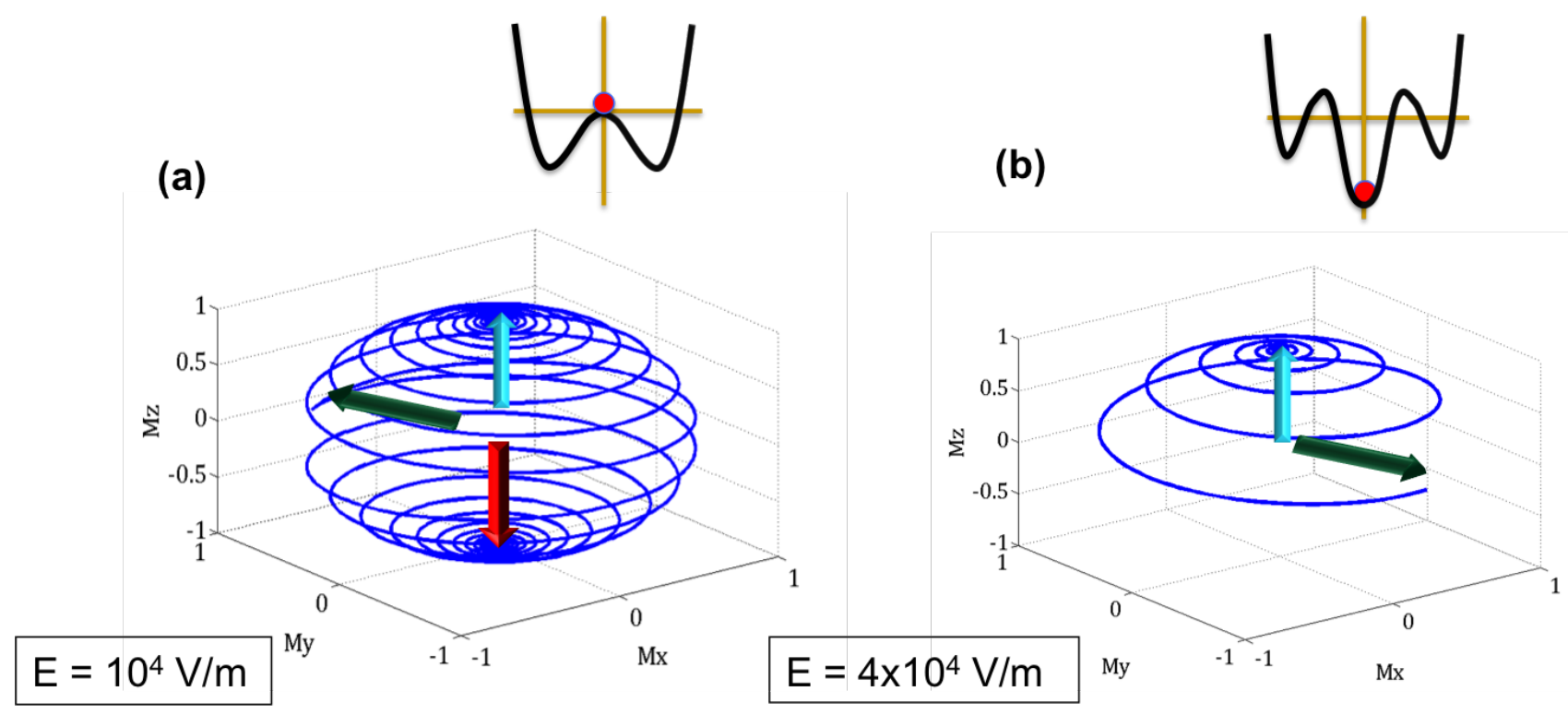

Figure 3.6: (a): The trajectory of magnetization in the central pillar in the presence of a weak electric field. The pillar first moves from the out-of-plane direction to the in-plane direction. When the electric field is turned off, the dipolar field causes the pillar to switch to the down magnetization. (b): With a strong applied electric field, the pillar switches to the in-plane direction and remains there when the electric field is turned off. The insets show the energy landscape for each of the scenarios.

in the inset) shows that this in-plane state is a metastable state. Any small magnetic field can tip the magnetization into the out-of-plane directions. The final regime occurs when the stress energy is large compared to the magnetostatic energies (Fig. 3.6(b)). When the electric field is strong, then the ferroelectric saturates and the remanent polarization keeps the magnetization in-plane. This is based on the energy landscape (see inset) having a global minimum in the in-plane direction. Furthermore, a large magnetic field is required to overcome the barriers and reach the out-of-plane state. The remanent polarization of the multiferroic allows for a persistent nulling of the ferromagnet even after the electric field is removed. With these three regimes of operation, we can encode information and perform logic operations. This will be described in the next section. 


\section{Chapter 4}

\section{Reconfigurable Array of Magnetic}

\section{Automata}

\subsection{Magnetic Quantum Cellular Automata}

Cellular automata (CA) were proposed by von Neumann as a possible computational paradigm [37]. They consist of binary state cells arranged in a regular grid, computation being performed through local interactions. It was later shown that cellular automata can be used to create a universal Turing machine capable of performing arbitrary logic [38]. Quantum Cellular Automata (QCA) have been implemented by using tunneling electrons to represent the binary cells [39], but the application of QCAs towards large scale systems has been hampered by the requirement of cryogenic temperatures. It has since been shown that Magnetic QCA (MQCA)which use the magnetization of nanomagnetsare better suited for low energy, room temperature operations [40]. Checkerboard MQCA cells (Fig 4.1(a)) can be arranged in various patterns to realize different gates such as inverters and majority gates, allowing them 
(a)

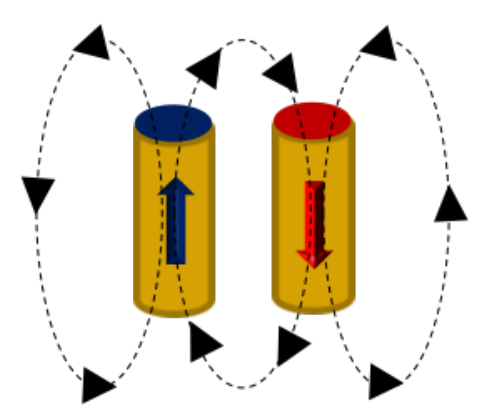

(b)

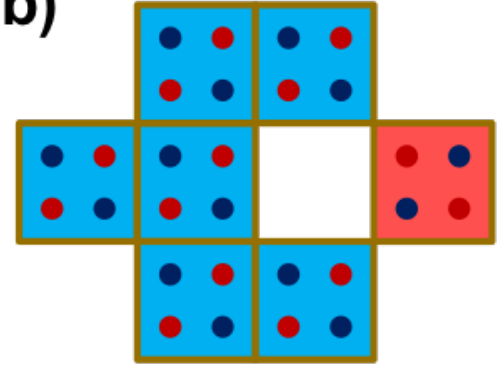

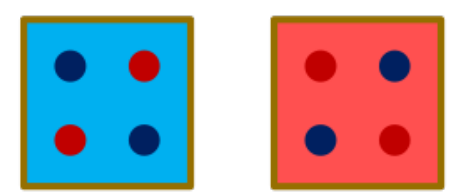

"0"

“1"

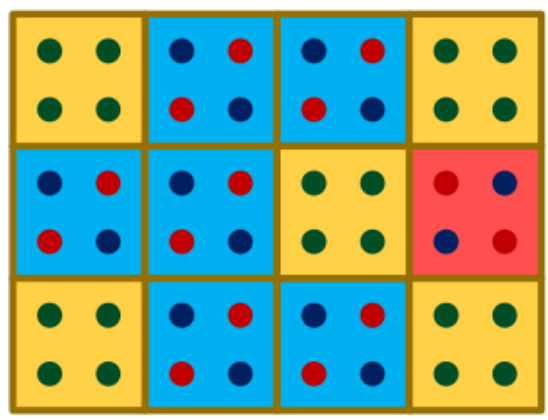

Figure 4.1: (a): Neighboring magnetic nanopillars prefer to align antiferromagnetically due to the dipolar exchange field. This creates two stable configurations which can be mapped as logic bit 0 and 1. (b): MQCA bit cells are patterned in different ways to implement various logic gates. Here an MQCA NOT gate is shown on the left. The same NOT gate is implement in RAMA as shown on the right. All unused bits are deactivated by placing the pillars into the in-plane direction.

to implement universal logic. One of the disadvantages of MQCAs is the need for localized magnetic fields to operate the devices. Therefore, we proposed using RAMA as a type of multiferroic MQCA that controls the magnetic properties by using electric fields [4, 41, 42].

\subsection{RAMA: A Multiferroic MQCA}

Reconfigurable Array of Magnetic Automata (RAMA) consists of a two-dimensional array of checkerboard MQCA bit cells (Fig. 4.2) [41, 42]. Each cell is multiferroic and made up of four ferromagnetic cobalt ferrite $\left(\mathrm{CoFe}_{2} \mathrm{O}_{4} ; \mathrm{CFO}\right)$ nanopillars which are immersed in a ferroelectric bismuth ferrite $\left(\mathrm{BiFeO}_{3}\right.$; $\left.\mathrm{BFO}\right)$ matrix. Changes to the magnetization of the 


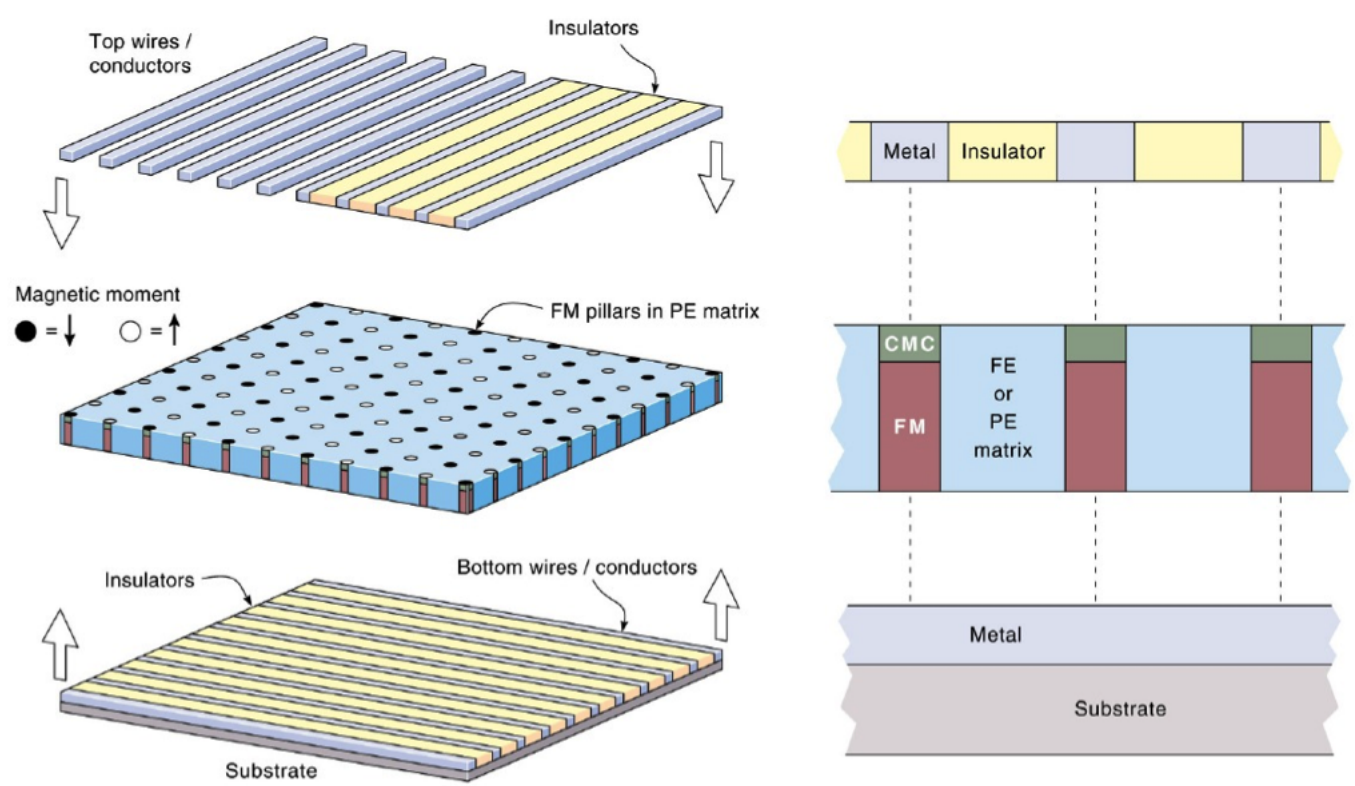

Figure 4.2: The structural layout of RAMA. The magnetic layer consists of ferromagnetic cobalt ferrite nanopillars (red) embedded in a ferroelectric bismuth ferrite matrix (blue). The magnetic layer is encased in a crossbar structure of wires. Single nanopillars can be selected by applying voltages to both the top and bottom metal layers just as a crosspoint switch.

CFO pillars are achieved by applying an electric field to the MQCA cell. The electric field changes the polarization of the ferroelectric $\mathrm{BFO}$ which in turn causes a change in its crystal structure. This structural change creates a strain on $\mathrm{BFO}$ which is transferred to the $\mathrm{CFO}$ nanopillars through the tight interfacial bonding between the two materials. Normally, the CFO magnetization prefers to be in the out-of-plane direction (up or down) perpendicular to the plane of the array. However, when a strong enough stress is applied to the CFO nanopillar, the preferred direction of magnetization rotates from out-of-plane to the in-plane direction. Depending on the magnitude of the applied electric field there are two cases possible depending on the magnitude of the electric field. 


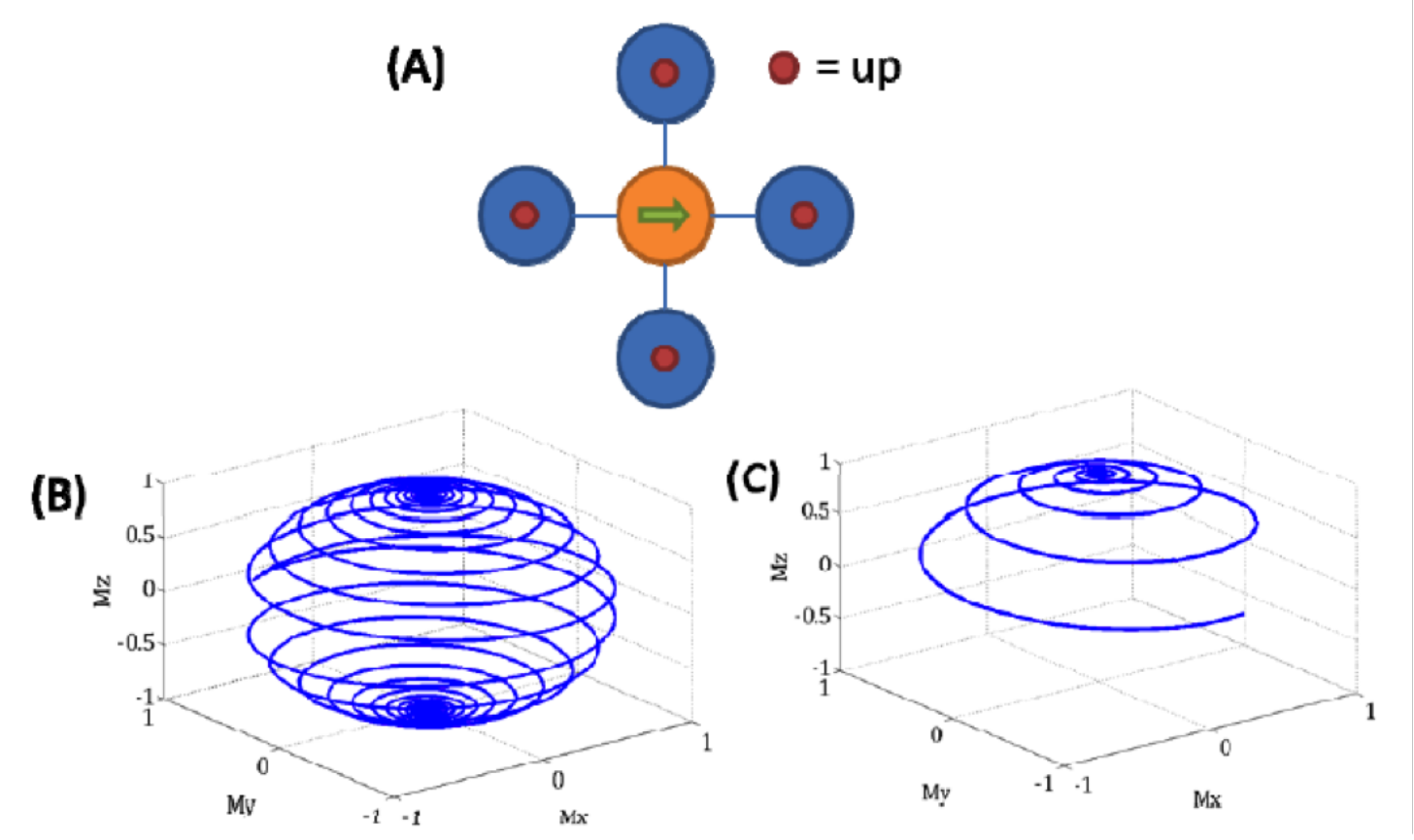

Figure 4.3: (a): The simulation setup includes a central pillar which is placed in the in-plane direction surrounded by neighboring pillars with fixed magnetizations in the up magnetization. (b): The trajectory of magnetization in the central pillar in the presence of a weak electric field. The pillar first moves from the out-of-plane direction to the in-plane direction. When the electric field is turned off, the dipolar field causes the pillar to switch to the down magnetization. (c): With a strong applied electric field, the pillar switches to the in-plane direction and remains there when the electric field is turned off.

\subsubsection{Reconfigurability}

If the electric field is strong, then the ferroelectric saturates and the remanent polarization (after the field is removed) keeps the magnetization in-plane - we use this property for configuring the array. The remanent polarization of the multiferroic allows for a persistent nulling of the magnetic nanopillars even after the electric field is removed. When the nanopillars are placed into the in-plane direction, the respective MQCA bits no longer interact with their neighbors. For example, Fig. 4.1(b) shows a typical pattern for a MQCA NOT gate (inverter). In RAMA, the patterning is done by reconfiguration, i.e. nulling the inactive cells. 


\subsubsection{Input \& Output}

On the other hand, if the electric field is relatively weak, the ferroelectric does not saturate, and when the electric field is removed there is little remanent polarization; thus, even as the magnetization still goes in-plane while the electric field is applied, it comes back out-of-plane when the electric field is removed - we use this property for clocking the array. Writing is performed by first clocking the nanopillars by placing them in-plane direction with a weak electric field and then applying a small biasing magnetic field in the direction to be written. Since neighboring nanopillars are coupled antiferromagnetically, the dipolar exchange field provides the biasing field to write to the appropriate state when the clock is removed. Reading of a nanopillar can be done inductively by placing a wire loop over the pillar - when the nanopillar is switched from the in-plane direction to the out-of-plane direction, the change in the magnetic flux in the wire loop induces a current through the loop. The direction of the magnetization determines the polarity of the current through the loop which can be used to read the bit. Other possible reading mechanisms are using colossal magneto-capacitance or magnetic tunnel junctions (MTJs).

\subsubsection{Half Select Problem}

The half select problem is an artifact of using a crossbar wiring structure. To configure a bit, half the voltage must be applied by the top wire and the other half by the bottom. However, this means that all the other MQCA bits on the same column and row feel half the activation voltage (Fig. 4.4(a)). This half-selected bit could possibly change the state of that bit.

However, the multiferroic properties of the MQCA can be used to ensure proper operations. 
(a)

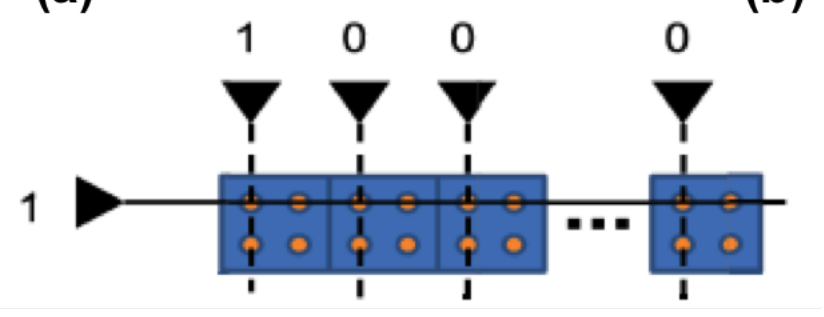

(b)

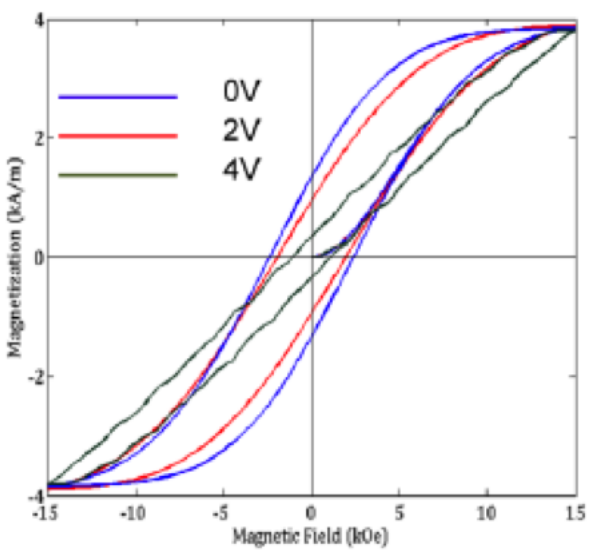

Figure 4.4: (a): When selecting a single bit, entire row and column of the crossbar structure feels at least half of the configuration voltage. (b): Magnetization hysteresis loops in the out-of-plane direction for various applied electric fields. The remananet magnetization $(\mathrm{H}=0)$ changes in a nonlinear manner when voltage is increased.

Because of the nonlinear nature of the material, changing the voltage by half does not necessarily mean that the bit is halfway between the out-of-plane diretion and the in-plane direction. This effect can be seen in Fig. 4.4(b). The change in the remnant magnetization is nonlinear when the applied voltage transistions between $0 \mathrm{~V}$ and $4 \mathrm{~V}$. Between $0 \mathrm{~V}$ and $2 \mathrm{~V}$, there is very little change in the remanance. However, the transition between $2 \mathrm{~V}$ and $4 \mathrm{~V}$ produces a much larger change in the remnant magnetization. Therefore, if $4 \mathrm{~V}$ was the configuration voltage, a half voltage of $2 \mathrm{~V}$ would not alter the state of the bit.

\subsection{RAMA Circuits}

The advantage of using a Verilog-A model is that it encapsulates the multiferroic physics of the nanopillar into a single circuit element which we can use to study more complicated scenarios. In the simulation shown in Fig. 4.3(a), we explore the interaction of a pillar with its neighbors. First, the central pillar is placed into the in-plane direction using electric 


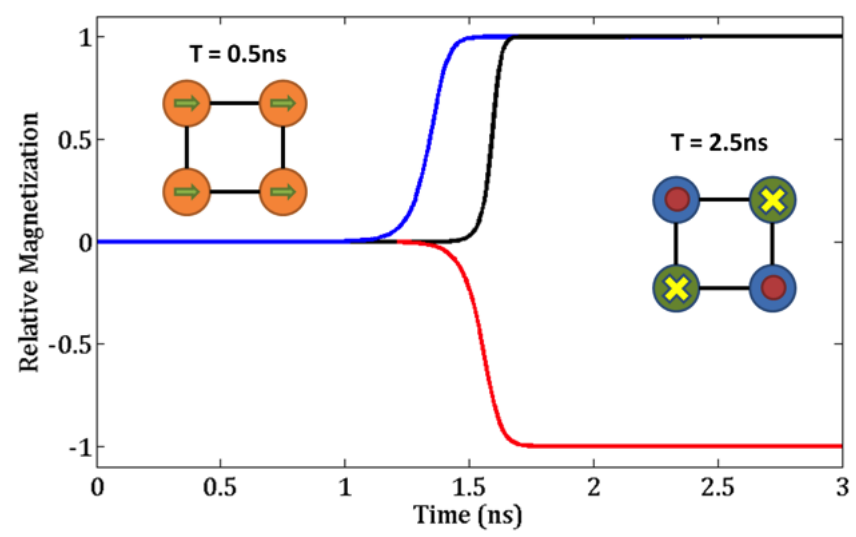

Figure 4.5: Verilog-A simulation of the initialization of an MQCA bit cell. The blue curve shows the evolution of the top left nanopillar, the red represents top right and bottom left pillars and the black curve represents the bottom right pillar.

fields of two different strengths - one that is comparable to the magnetostatic energies and one that is much larger. The magnetizations of the neighboring pillars are kept fixed in the out-of-plane direction - then we turn off the applied electric field and allow the pillars to interact through their dipolar exchange fields. We find that in the comparable electric field scenario, the pillar is switched into the out-of-plane direction and antiferromagnetically aligned with its neighbors (Fig. 4.3(b)) - thus performing a write operation. However, when the electric field is large (Fig. 4.3(c)), the dipolar exchange energy cannot overcome the stress energy from the remanent polarization even when the electric field is turned off. Therefore, the magnetization remains in the in-plane direction. This mode of operation is useful during the configuration phase in RAMA where we deactivate cells to prevent them from interacting with neighboring cells. In order to remove the in-plane magnetization, a reverse electric field is needed to restore the out-of-plane magnetization. Thus the strength of the electric field determines whether we are clocking the device for read/write operation or whether we are configuring the array. We also used the Verilog-A model to simulate the behavior of some 

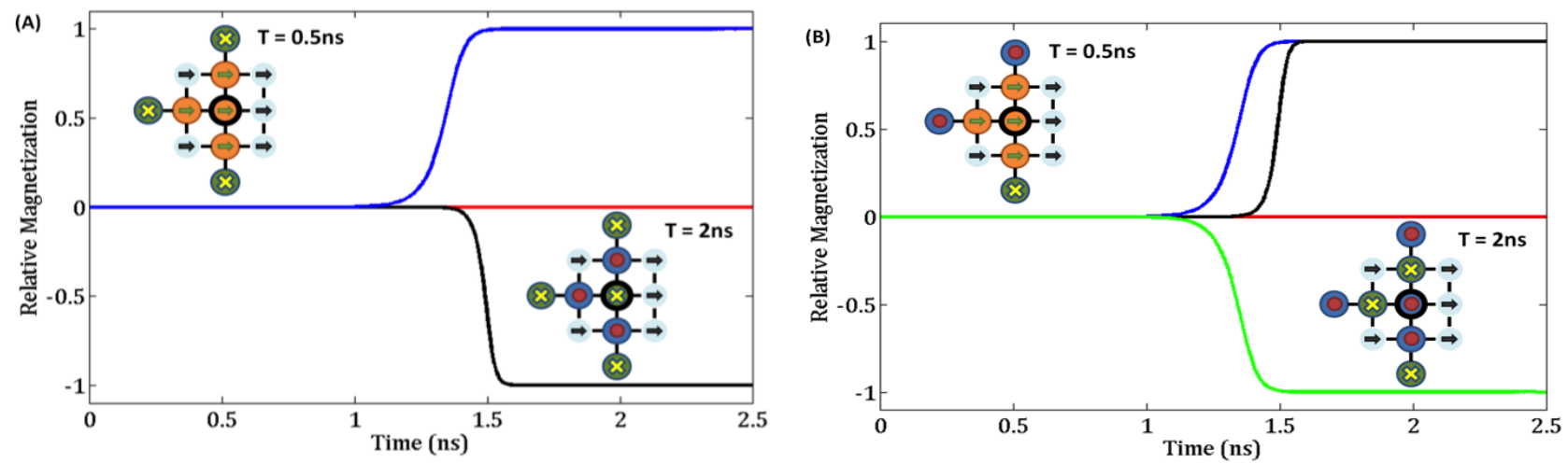

Figure 4.6: Simulation of MQCA majority gate. The black curve represents the state of the output bit shown with the bolded outline in the diagrams. The red line represents the state of the configuration bits, while the other curves represent the state of the other bits. (a): Dynamics of majority gate when all inputs are the same. (b): Outputs when one input is different from the others.

common structures in MQCA circuits. In simulating these structures, we only considered nearest neighbor dipolar interactions (higher order interactions can be added to the model by including extra terms in the LLG equations for second neighbors, etc.). In Figure 4.5, we simulate the checkerboard pattern of the MQCA bit cell which is an important requirement for proper operations in RAMA. First, we clock all four pillars into the in-plane direction. Then we bias the top left corner pillar using neighboring pillars (not shown) and allow the other pillars to evolve through their mutual interactions. Initially, the top left corner cell switches to the out-of-plane direction. This transition affects the two nearest neighboring pillars and causes them to move to the antiparallel alignment. Finally, these two pillars cause the last pillar to switch into the checkerboard formation. A structure that is commonly used in MQCA circuits is the majority voter (MV) gate which can implement other logic gates such as AND or OR gates. Using the Verilog-A multiferroic model, we implemented a majority voter gate with three input cells (with fixed magnetization) and one output bit. The central structure is made up of a $3 \times 3$ tile of nanopillars with the corner cells deactivated 
in order to demonstrate the configuration process. In Figure 4.6, we illustrate the outputs of the active central pillars and the output bit for two sets of inputs.

Having demonstrated some of the basic gates using multiferroic nanopillars, we can now look at an architecture for RAMA which can implement large scale, arbitrary logic.

\subsection{RAMA Architecture}

In this section we look at a circuit architecture for RAMA which can realize large scale logic. Our criteria for a successful architecture must be able to:

- Implement arbitrary logic functions using simple primitives like majority voter (MV) gate and inverters

- Take advantage of the reconfigurability of RAMA to create an FPGA-style programmability to maximize the benefits of the multiferroic technology.

- Break the inherent symmetry of MQCA gates and show unidirectional propogation of information from input to output.

Given our requirements for the RAMA logic system, the best architecture which addresses all these specifications has been the work of Huang et. al. [43, 44]. In the following sections we give a description of this architecture and demonstrate simple circuits using this methodology.

\subsubsection{MQCA Tiling Logic}

Consider the processing features of CMOS systems: some circuits perform computation, while others are used for signal/data transfer and communication. In FPGAs, for example, 


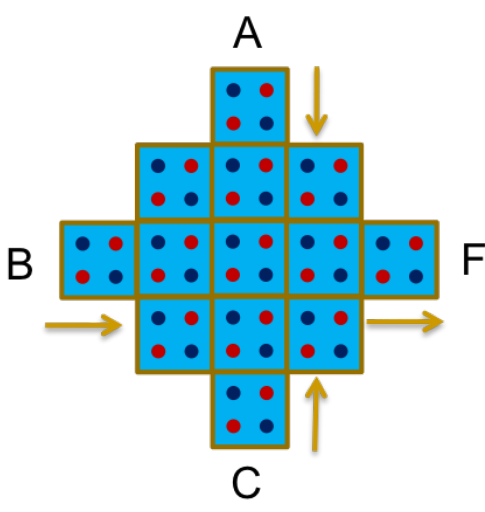

(a)

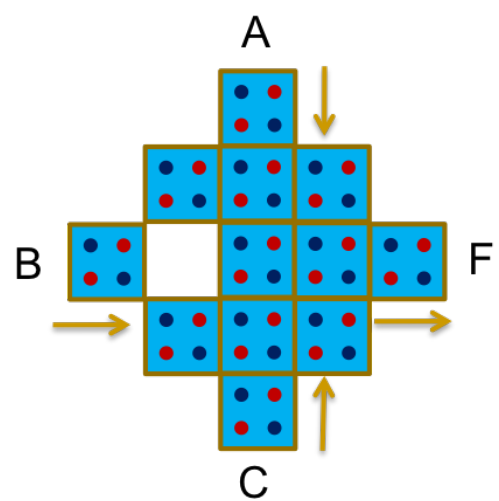

(b)

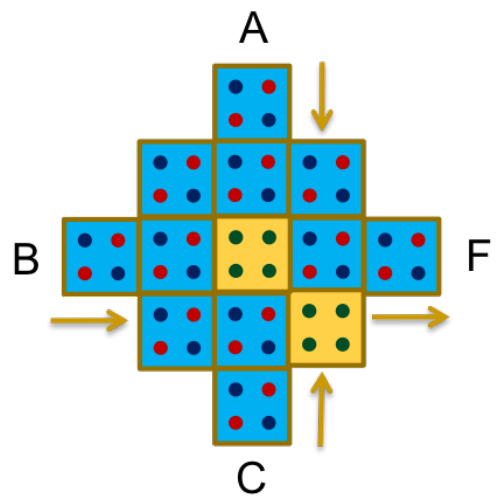

(c)

Figure 4.7: (a): A 3x3 fully populated (FP) majority voter (MV) gate. (b-c): By selectively deactivating cells in the $3 \times 3$ grid, different logic gates can be realized. In RAMA, this is achieved by placing the cell in the in-plane configuration state.

computation is performed by the logic resources or processing elements, while communication is accomplished by the interconnect fabric. In MQCA, computation and communication occur simultaneously. Logic elements are based on two gate primitives-the inverter, INV (Fig. 4.1(b)) and majority voter gate, MV (Fig. 4.6) -to implement combinational circuits; as for interconnect, a chain of MQCAs referred to as the binary wire is utilized.

In this architecture, a design based on elementary building blocks referred to as tiles, is proposed for MQCA. Two types of tiles, namely the active tiles and the passive tiles can be used. A tile is defined as active if it implements a combinational logic function with minterms of at least two literals. For example, a tile that performs the majority voter function is an active tile. A tile is said to be passive if it implements logic functions of only one literal, that is, wire and INV functions. The basic logic primitive in the proposed design is the MV-like tile. This tile performs a majority function with selective inversion (if required) at its inputs. The MV-like function is universal and area efficient compared with using separate MV and INV. In addition to the MV-like tile, passive tiles are used for interconnect. 
A tile can be built from an $n \times n$ square grid of MQCA cells. Both fully populated (FP) and non fully populated (NFP) grids can be used as basic logic blocks. Fig. 4.7(a) illustrates a $3 \times 3 \mathrm{FP}$ grid made up of 9 cells with three inputs (A, B and C) and one output (F). An NFP grid (Fig. 4.7(b)) is obtained in RAMA from the FP grid by selectively deactivating the cell by using a strong electric field and placing the pillars in the stable in-plane configuration (see e.g. Fig. 3.6(b)).

Among $n \times n$ grids, the $3 \times 3$ grid has computational properties which makes it very attractive for designing larger circuits. Tiles which utilize the $3 \times 3$ grid, are therefore used as examples. Using different input and output cells, these tiles are analyzed as providing a high degree of flexibility in logic operation. Different logic functions can be generated by using less than $n^{2}$ cells in a grid of dimension of $n$ (NFP grid) [45]. The functional behaviors of the tiles are reported based on simulations using the Verilog-A model.

\subsubsection{Logic Primitives \& Clocking}

One of the primitive logic gates in MQCA is the majority voter. The majority voter with logic function $\operatorname{Maj}(A, B, C)=A B+A C+B C$, can be realized using MQCA cells, as shown in Fig. 4.6. Logic AND and OR functions can be implemented from the majority voter by setting one of the inputs (the so-called programming or control input) permanently to a 0 or 1 value, respectively. The inverter is the other basic gate in MQCA and is shown in Fig. 4.1(b).

In MQCA, timing is accomplished by clocking in four distinct and periodic phases (as shown in Fig. 4.8). A MQCA circuit is partitioned into a number of clocking zones and all 


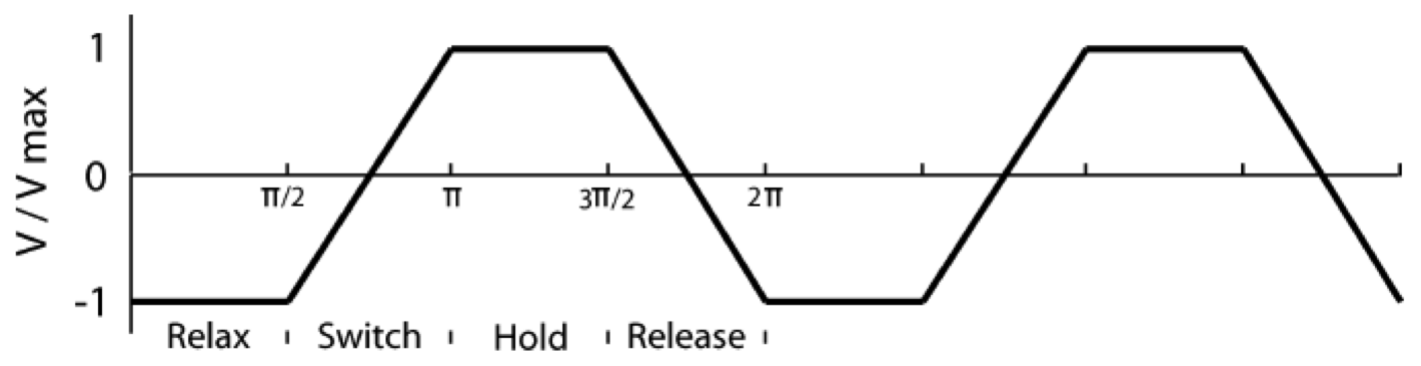

Figure 4.8: Four-phased signal for clocking zones in MQCA, adiabatic switching.

cells in the same zone are controlled by the common clock signal. The use of a quasi-adiabatic switching technique for MQCA circuits requires a four-phased clocking signal for modulating the magnetic barriers between the bi-stable magnetization states. This is done using a small electric field as shown in Fig. 3.6(a), and allowing the neighboring dipolar interaction or small biasing field to set the new state.

The four phases of the clock are Relax, Switch, Hold and Release (Fig. 4.8). During the Relax phase, there is no electric field and a cell remains in one of the bi-stable out-of-plane states. During the Switch phase, the electric field is slowly raised and the cell is placed in the metastable in-plane state. In the Hold phase, the cell feels the interactions of its neighboring cells (or small external biasing field), causing it to move away from the metastable in-plane states to one of its bi-stable states. Finally, in the Release phase, the electric field is removed and the cell remains in its stable state. Timing zones of a MQCA circuit or system are arranged in this periodic fashion such that zones in the Hold phase are followed by zones in the Switch, Release and Relax phases.A signal is effectively latched when one clocking zone goes into the Hold phase and acts as input to the subsequent zone. This clocking mechanism provides inherent pipelining and directionality and allows multibit information transfer for MQCA through signal latching. 

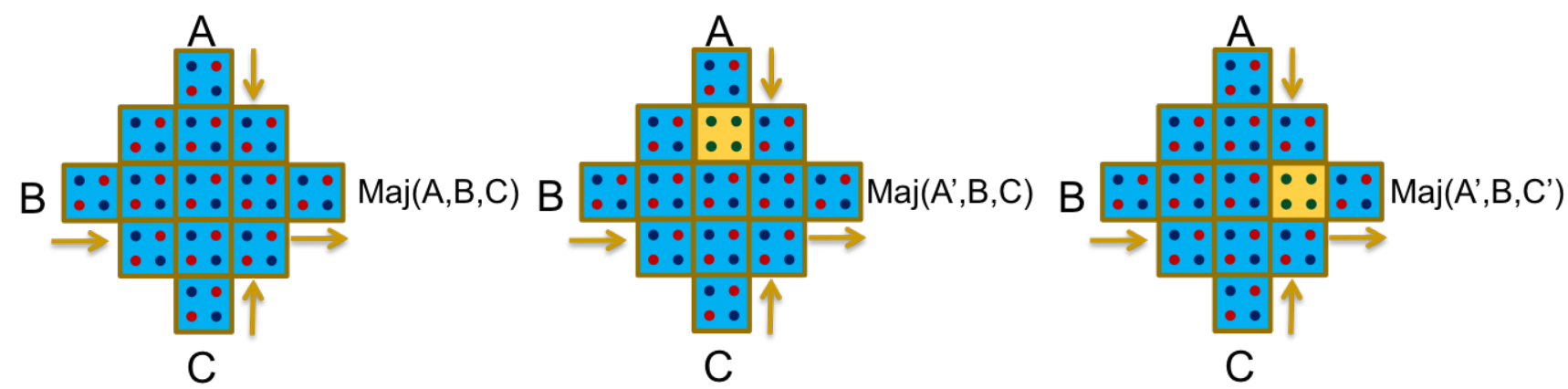

Figure 4.9: Different configurations for the orthogonal tile. By deactivating cells in the orthogonal tiles, the majority logic can be changed by inverting the inputs depending on the configuration.

\subsection{3 $3 \times 3$ MQCA Tiles}

An NFP grid is generated from an FP grid by selectively deactivating cells causing changes in the logic behavior of a MQCA circuit. So, it is interesting to compare the characteristics of tiles with different input/output cells. For the $3 \times 3$ grid, several different tiles are possible. Tiles with one input and one output are not considered due to the obvious wire function; they are referred to as interconnection (passive) tiles. The other scenarios are considered below:

- The orthogonal tile has three inputs (the horizontal input cell B and the vertical input cells A and C) and one output (the horizontal output cell F), as shown in Fig. 4.9(a). New MV-like functions (majority function with at least one input inversion) and NXOR are possible at the output by selectively deactivating cells in this tile (Fig. 4.9(b-c)). The MV-like function occurs due to the interaction of the cells at the corners of the tile with the center cell of the MV.

- The double and triple fan-out tiles are shown in Fig. 4.10(a) \& (b); these tiles have one input (given by the horizontal cell B) and two (or three) outputs (given by the output cells F1, F2 (and F3)). Simulation results show that both original and 


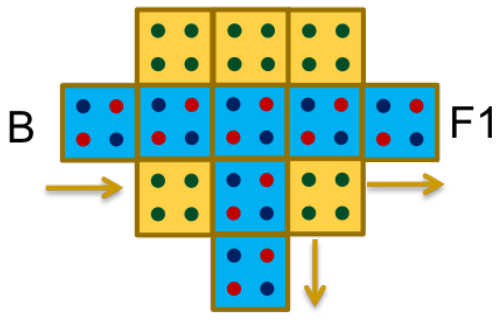

F2

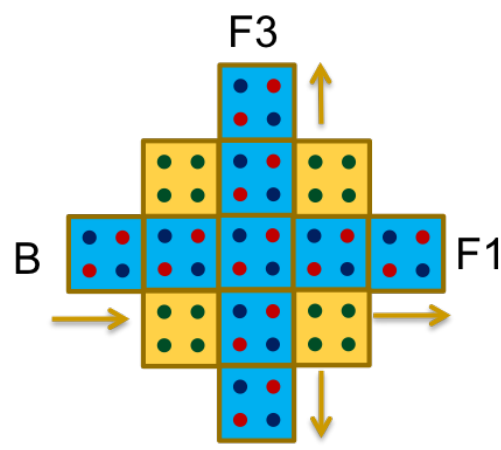

$\mathrm{F} 2$

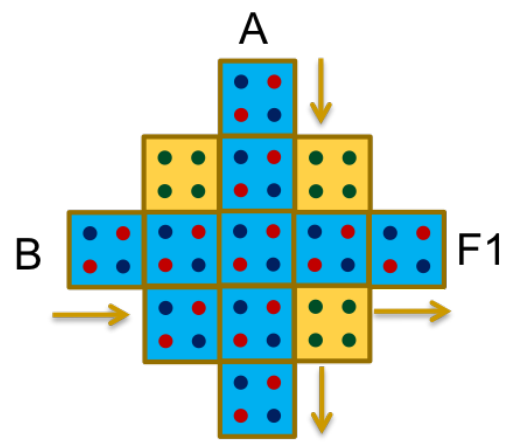

$\mathrm{F} 2$

(a)

(b)

Figure 4.10: Addition structures in MQCA tiling logic. (a): Double fan-out with one input and two output. (b): Triple fan-out with one input and three outputs. (c): Baseline gate which implements wire crossings with two inputs and two outputs.

complemented signals can be observed at the outputs by deactivating the appropriate cells in these tiles.

- The baseline tile has two inputs (one vertical input cell A and one horizontal input cell B) and two outputs (the horizontal output cell F1 and the vertical output cell F2), as shown in Fig. 4.10(c). This tile accomplishes wire crossing and signal complementation by selectively deactivating the appropriate cells.

With these logic tiles, we can implement any arbitrary logic. In the next section, we use the tile primitives to implement a single-bit full adder as a proof of concept of circuit design.

\subsubsection{Single-bit Full Adder}

In this section, the single-bit full adder is analyzed. In this design, different tiles such as the baseline, double and triple fan-out and the orthogonal tiles are utilized. The baseline tile is used to achieve wire crossing; the double and triple fan-out tiles are used for signal routing; MV as well as MV-like functions are employed using the orthogonal tile. It is interesting that 

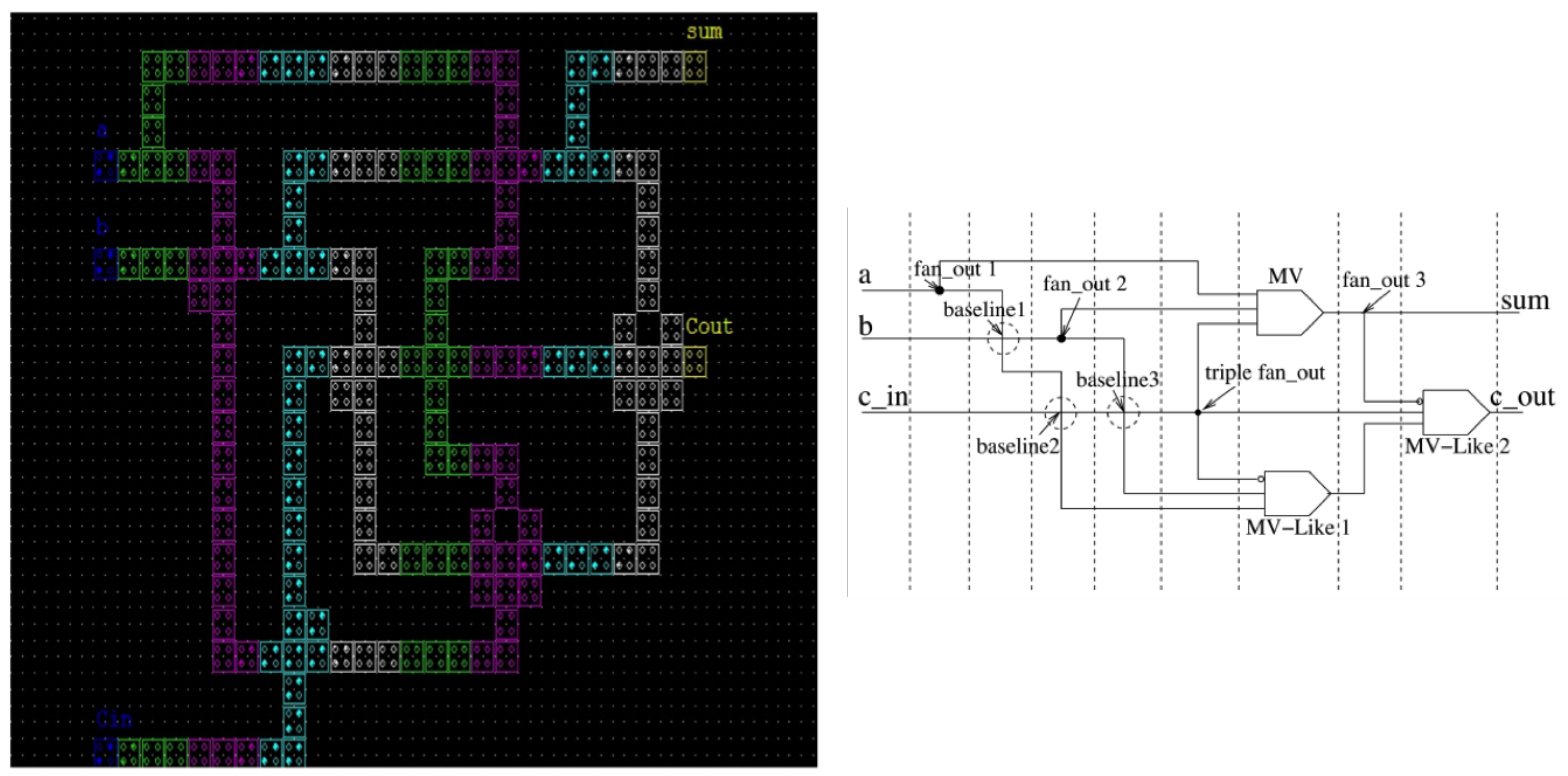

Figure 4.11: Implementation of single-bit full adder and its corresponding circuit diagram.

although the MV and the triple fan-out are similar, the arrangements in input/output cells cause the two tiles to function differently. Also the flow of signals is enforced by arranging the clocking zones.

The single-bit full adder is built using one MV and two MV-like (with inversion at one of the inputs) gates. The MQCA layout as well as the corresponding circuit schematic are shown in Fig. 4.11. Three baseline tiles (as wire crossing), two double fan-out tiles, one triple fan-out tile, three orthogonal tiles (as MV and MV-like) are used in this design. These tiles are connected using passive tiles, which function as wires. The MV gate and the MV-like gates are highlighted by dotted squares. In the design of a full adder, no additional isolation is needed between tiles. The waveforms for the single-bit adder are shown in Fig. 4.12. 


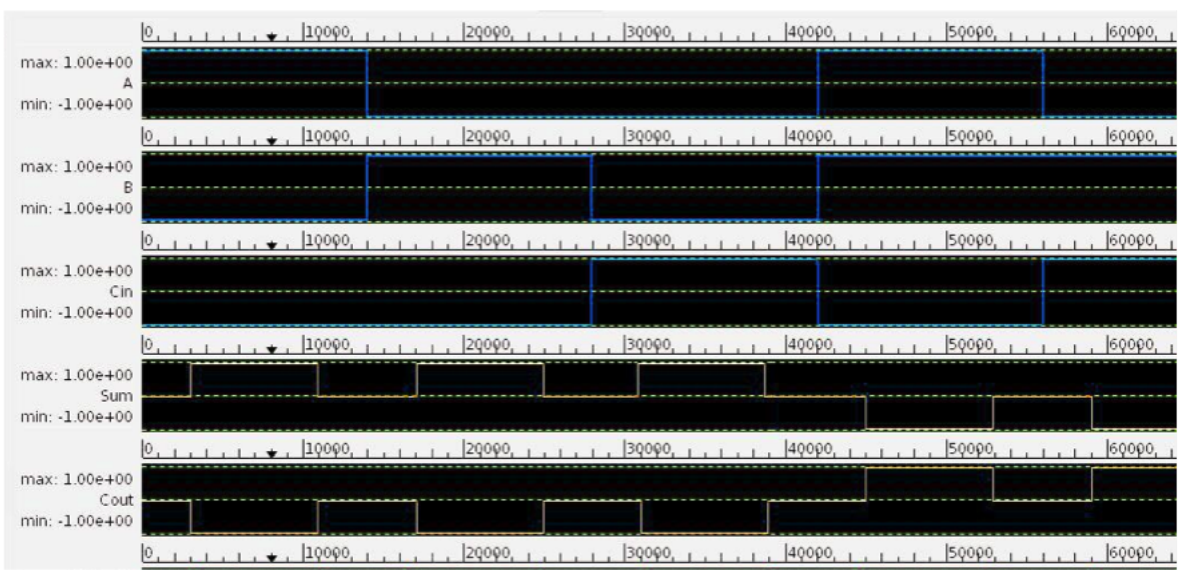

Figure 4.12: Waveform output of a single-bit full adder.

\subsection{RAMA Benchmarking}

The unique computational architecture of RAMA makes it an attractive possible post-CMOS technology. The main advantage of RAMA comes from the low powered switching and the correlated states between neighboring pillars which aids in lowering the energy barrier during the switching process. In addition, the ordered array structure allows for both non-volatile memory and reconfigurable logic on the same chip.

The disadvantage of RAMA comes from the relatively slow switching speed $\left(\sim 10^{-10}\right.$ seconds) compared to the latest technology node MOSFETs. This is due to the response time of the ferroelectric material to the applied electric field. Therefore, RAMA would not be ideal for high performance computation that are the focus of novel post-CMOS technologies (e.g. graphene)

In order to benchmark RAMA with other post-CMOS technologies, an effort was made [46] to derive similar metrics for various nanotechnologies in implementing a two-input NAND gate. This benchmarking effort took into account the full process of computation, from application of electric fields to clocking of the logic gates. The results for the energy vs. delay 


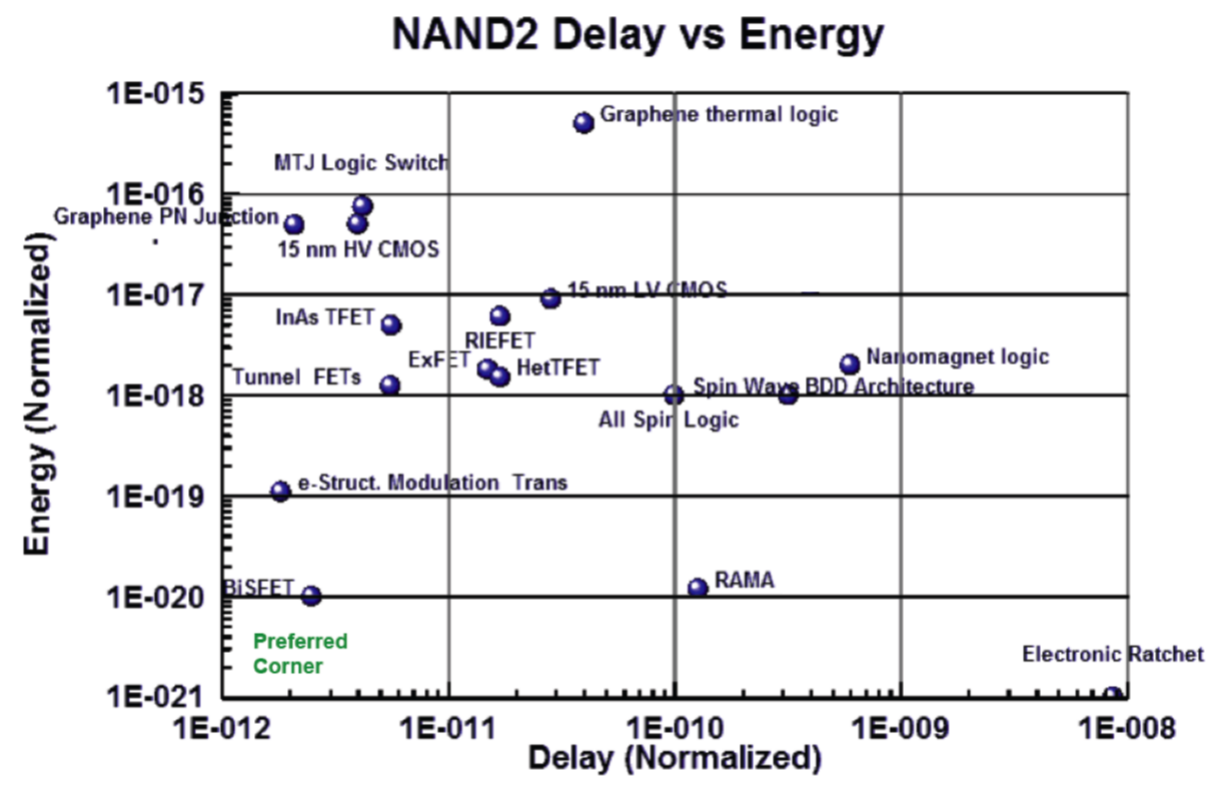

Figure 4.13: Comparison of the energy and delay of a 2-input NAND gate in RAMA versus other emerging technologies.

are shown in Fig. 4.13. Based on these results, RAMA would be ideal for ultra-low power applications requiring long battery life and modest computational intensity. 


\section{Chapter 5}

\section{Modeling of Spin-Torque}

\section{Nano-Oscillators}

The operation of spin torque oscillators takes advantage of two key discoveries in magnetic materials-spin dependent transport and spin transfer torque. These discoveries were made in a structure known as the magnetic tunnel junction (MTJ), which consists of two ferromagnetic metal layers separated by a thin insulating barrier layer (Figure 5.1). The insulating layer is so thin (a few nm or less) that electrons can tunnel through the barrier when a bias voltage is applied between the two metal electrodes. An important property of an MTJ is that the tunnelling current depends on the relative orientation of the magnetizations of the two ferromagnetic layers, which can in turn be changed by an applied magnetic field. This phenomenon is called tunneling magnetoresistance (TMR). In this section, we review some of the major results which led to current focus on spin torque nano-oscillators. We will then develop the dynamical equations for the Verilog-A model and an analytical model which can accurately explain the oscillation frequency and synchronization of multiple oscillators. 
After validating the results with experiment, we look at the geometry of the spin torque nano-oscillator and its consequence for synchronization in a parallel electrically-coupled array.

\subsection{Spin Dependent Transport}

The history of spin-dependent transport in magnetic multi-layers went from obscurity to Nobel Prize winning research. Here we present just a brief outline of that history-full reviews of the research progress are given in the following excellent papers [47, 48, 49, 50, 51]. The first work on spin dependent transport began more than 40 years ago with experiments by Meservey et. al. $[52,53]$. A few years earlier, the BCS theory of superconductivity had been published [54] and there was great interest in transport properties of electrons through superconducting junctions [55]. Meservey et. al. predicted that the energy level of a superconductor in a magnetic field could be split in such a way that it could act as a high precision spin polarization detector. This allowed them to constructed a trilayer junction, consisting of ferromagnetic-insulating-superconducting layers, which could detect the conduction changes due changes in the magnetization orientation of the ferromagnetic layers. This was the first experimental verification that electron transmission could be a function of its spin orientation.

Soon afterwards, Julliere [56] demonstrated tunneling magnetoresistance (TMR) between two ferromagnetic layers separated by an insulating barrier. By using ferromagnets of different coercivities, it was possible to detect the change of resistance between parallel magnetizations and anti-parallel magnetizations. Julliere postulated that the TMR could be calculated based on the spin polarization of the two ferromagnetic layers as given by the equation: 

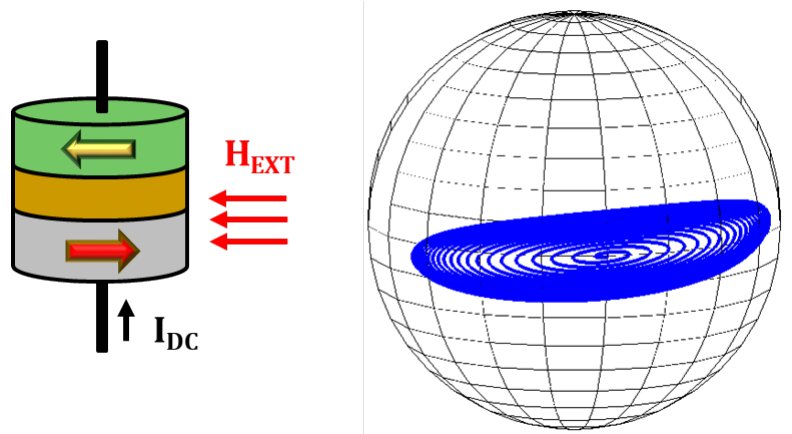

Figure 5.1: (a): Structure of a magnetic tunnel junction with a fixed layer, whose magnetization is pinned, and a free layer, whose magnetization can be changed. (b): The stable precession trajectory of the magnetization of an in-plane free layer in response to spin transfer torque.

$$
T M R=\frac{2 P_{1} P_{2}}{1-P_{1} P_{2}}
$$

where $P=\frac{\rho_{\uparrow}-\rho_{\downarrow}}{\rho_{\uparrow}+\rho_{\downarrow}}$ and $\rho_{\uparrow}$ and $\rho_{\downarrow}$ are up-spin and down-spin electron carriers, respectively, in the ferromagnetic layer.

Despite these discoveries, there would be very little research on spin-dependent transport for another decade. Partly this was caused by technologically demanding fabrication process, which makes it difficult to fabricate robust and reliable tunnel junctions. Also, the fact that the reported values of TMR were small (at most a few percent at low temperatures), did not trigger considerable interest in view of sensor/memory applications. The perfecting of molecular beam epitaxy (MBE) and its greater availability during the 1980s [57] allowed for better thin-film fabrication allowing studies of magnetic thin-film structures.

The discovery of giant magnetoresistance (GMR) in spin-valve structures (using metallic spacer layers) ignited interest in spintronic devices. Because of their pioneering work on GMR, Albert Fert [5, 58] and Peter Grünberg [6] were awarded the Nobel Prize in Physics in 2007 [50, 51]. While previous experiments used cryogenic conditions, room temperature 
operations were shown by Miyazaki and Tezuka $[59,60]$ that demonstrated the possibility of large values of TMR in MTJs with $\mathrm{Al}_{2} \mathrm{O}_{3}$ insulating layers, while Moodera et al. [61] developed a fabrication process, which appeared to fulfill the requirements for smooth and pinhole-free $\mathrm{Al}_{2} \mathrm{O}_{3}$ deposition.

The first accurate theoretical consideration of TMR was made by Slonczewski in 1989 [62]. He considered tunnelling between two identical ferromagnetic electrodes separated by a rectangular potential barrier assuming that the ferromagnets can be described by two parabolic bands shifted rigidly with respect to one another to model the exchange splitting of the spin bands. By imposing perfect translational symmetry of the tunnel junction along the layers and matching the wave functions of electrons across the junction, he solved the Schrödinger equation and determined the conductance as a function of the relative magnetization alignment of the two ferromagnetic films. In the limit of thick barrier, he found that the conductance is a linear function of the cosine of angle $\theta$ between the magnetic moments of the films:

$$
G(\theta)=G_{\text {int }}\left(1+P^{2} \cos \theta\right)
$$

where $G_{\text {int }}$ is the normal conductance through the trilayer interfaces, $P$ is the spin-polarization of the fixed layer and $\theta$ is the angular difference in the magnetic orientation between the fixed and free layers. This matches the previous observations in Julliere's equation (Eqn. 5.1) that parallel magnetizations have higher conductance than anti-parallel orientation. 


\subsection{Spin Transfer Torque}

The other effect, spin transfer torque, was first predicted by Slonczewski [63] and Berger $[64,65]$ and subsequently verified experimentally in oxide-based MTJs $[66,67]$. This behavior is observed in MTJs when a DC current is applied through the junction. One of the ferromagnetic layers (known as the fixed or pinned layer) is used to spin-polarize the DC current to align a majority of electrons in the spin orientation of layer. When these electrons tunnel through the insulating layer and are injected into the second ferromagnetic layer (the free layer), they transfer their angular momentum to electrons in the free layer causing the magnetization to change. Normally, dynamic processes in magnetic layers create damped oscillations which dissipate over time. However, Slonczewski showed that the spin transfer torque effect can be used to cancel out the dampening effect, creating stable oscillations, and in some cases, complete reversal of the magnetization [68] (Figure 5.1(b)). The latter effect can be used to perform the "write" operation of MTJs in magnetic memory devices such as MRAM and STT-RAM [69, 70], while the former can be used to create microwave oscillations in spin torque nano-oscillators (STNO).

Soon after the theoretical development, spin torque nano-oscillators were demonstrated experimentally [68, 71, 72]. The microwave oscillations generated by the STNOs exhibit wide ranging tunability simply by adjusting the DC current and applied magnetic field (Figure 5.1(a)), with frequencies ranging from $0.1 \mathrm{GHz}-100 \mathrm{GHz}$ and linewidths on the order of $\sim 100 \mathrm{MHz}[73]$. More recently, it has been experimentally shown that two STNOs can mutually phase-lock and synchronize [74, 75]. These experiments involved two MTJ structures with a shared free layer which allowed current injected spinwaves to interact with each other 
causing them to phase-lock. The ability of spin torque oscillators to synchronize is not only advantageous in increasing the output oscillation power [76] but also provides a way of transferring information and computing with multiple oscillators.

In this paper, we propose an array of STNOs which can perform Non-Boolean computations through synchronization. Specifically, we show how to use the STNO array for pattern recognition, which serves as a first step towards associative memory behaviour [77, 78]. Throughout this paper, we discuss some of the design parameters that have to be considered for effective synchronization of the oscillators. In Sections 5.3-5.5, we discuss the dynamics of the STNO and how electrically coupling them can provide an alternative method to synchronization. In Section 5.6, we examine the device geometries which are optimal for coherence of multiple STNOs and introduce the idea of parallel connected DMTJ-STNO arrays. We use HSPICE simulations to model the spin torque nano-oscillator behavior. More details about the simulation setup are provided in the Appendix.

\subsection{Modeling the Dynamic Behavior of STNOs}

The precessional motion of magnetization (M) of the free layer of an MTJ, in the presence of an effective magnetic field $\left(\mathbf{H}_{\mathbf{e f f}}\right)$, can be accurately modeled by the Landau-Lifshitz-Gilbert (LLG) equation (Eqn. 2.15). With the introduction of Slonczewski's spin-transfer torque, the LLG equation with the STT term is given by:

$$
\frac{\partial \mathbf{M}}{\partial t}=-\gamma_{0} \mathbf{M} \times \mathbf{H}_{\mathrm{eff}}+\frac{\gamma_{0} \alpha}{M_{s}} \mathbf{M} \times\left(\mathbf{M} \times \mathbf{H}_{\mathrm{eff}}\right)+\eta(\theta) \frac{\mu_{B} I}{e V} \mathbf{M} \times\left(\mathbf{M} \times \mathbf{M}_{\mathbf{p}}\right)
$$


The first term in Eqn. (5.3) is the precession of the magnetization (M) around and effective magnetic field $\left(\mathbf{H}_{\text {eff }}\right)$ where $\gamma_{0}$ is the gyromagnetic ratio. The second term describes the phenomenological dampening $(\alpha)$ which brings the magnetization in anti-/parallel alignment with $\mathbf{H}_{\text {eff }}$ (here $M_{s}$ is the saturation magnetization of the free layer). Finally, the third Slonczewski term is similar to the dampening term, except the torque pulls the magnetization towards the orientation of the polarizing layer $\left(\mathbf{M}_{\mathbf{p}}\right)$. The spin torque is modified by the applied current density given by $\left(\frac{I}{V}\right)$, where $V$ is the free layer volume, and also by the spin polarization efficiency factor $\eta(\theta)$ given by [63]:

$$
\eta(\theta)=\left[-4+(1+P)^{3} \frac{3+\cos \theta}{4 P^{3 / 2}}\right]^{-1}
$$

where $P$ is the polarization of the fixed layer and the angle $\theta$ is the relative orientation between the free and fixed layer.

The effective magnetic field $\left(\mathbf{H}_{\mathbf{e f f}}\right)$ is given by:

$$
\mathbf{H}_{\mathrm{eff}}=\mathbf{H}_{\mathrm{ext}}+\mathbf{H}_{\mathrm{an}}+\mathbf{H}_{\mathrm{dem}}+\mathbf{H}_{\mathrm{amp}}
$$

where $\mathbf{H}_{\text {ext }}$ is the external applied magnetic field. The field $\mathbf{H}_{\mathbf{a n}}$ represents the magnetocrystalline anisotropies that exist due to the crystal structure. This anisotropy contribution can be further divided into contributions from the easy-axis, perpendicular axis and planar fields:

$$
\mathbf{H}_{\mathrm{an}}=\mathbf{H}_{\mathrm{easy}}+\mathbf{H}_{\text {perp }}+\mathbf{H}_{\text {planar }}
$$

The easy axis field lies in the plane of the free layer while the perpendicular axis is the 
out-of-plane contribution. Finally, the planar field is the field that tries to pull an out-of-plane magnetization towards the free layer plane. The demagnetization field captures the shape anisotropy of the magnetic material and depends on the geometry. In general, $\mathbf{H}_{\mathbf{d e m}}=\mathbf{N} \cdot \mathbf{M}$, where $\mathbf{N}$ is a shape dependent term [10]. Finally, $\mathbf{H}_{\mathbf{a m p}}$ is the amperian field created by the injected current within the magnetic tunnel junction. This circular field will lie in the plane of the free layer and will depend on the magnitude of the current through the wire.

\subsection{Self-oscillation in STNOs}

\subsubsection{Universal Oscillator Model}

In this section, we will develop a theory for self-oscillation of spin torque nano-oscillator based on the Verilog-A model. This theory will help model STNOs and explain how they maintain the precession and achieve synchronization. In this respect, we follow the reviews by Slavin et. al. [79] and Li et. al. [80] which provide the standard expositions on the subject.

Self-oscillatory systems are very common in nature and technology [81, 82, 83]. Despite of a wide variety of different physical realizations, all self-oscillatory systems have the same three key elements: (i) resonant element that determines frequency of self-oscillations, (ii) dissipative element, which is inevitable in any real device or mechanism, and (iii) active element that compensates the energy losses and makes self-oscillations possible. In many cases the effect of the active element can be described as a negative damping, i.e., the presence of an active element in the system is reflected in the dynamical equations of motion by the presence of a term that has a form, similar to the term, which describes the natural damping, 
but with opposite (negative) sign.

The majority of self-oscillators of this type, regardless of a particular physical realization, can be described by the same nonlinear oscillator model [79]:

$$
\frac{d c}{d t}+i \omega(p) c+\Gamma_{+}(p) c-\Gamma_{-}(p) c=f(t)
$$

or the complex amplitude $c(t)$ of self-oscillations, which measures both the power $p=|c|^{2}$ and phase $\phi=\arg (c)$ of the oscillations. In Eqn. 5.7, $\omega(p)$ is the resonance frequency of the resonant element, $\Gamma_{+}(p)$ is the damping rate for the natural energy dissipation (natural, or positive, damping), negative damping rate $\Gamma_{-}(p)$ describes the effective action of the external or internal energy source (active element), and the driving term $f(t)$ describes the interaction of the self-oscillator with the rest of the world, e.g., influence of external signals or/and thermal fluctuations. The resonance frequency $\omega(p)$ and both damping rates $\Gamma_{ \pm}(p)$ are, in general, functions of the self-oscillation power $p=|c|^{2}$, which makes the self-oscillator a nonlinear dynamical system.

We consider the simplest possible geometry of spin-torque nano-oscillator (STNO)the oscillator based on a normally magnetized isotropic magnetic nano-pillar shown schematically in Fig. 5.2. We will assume that the spin-polarized current is uniform across the area of the free layer and that only the spatially-uniform magnetization precession is excited. This simple case allows one to perform reduction of Eqn. 5.3 to the universal oscillator model Eqn. 5.7 exactly, and without complicated mathematics. 


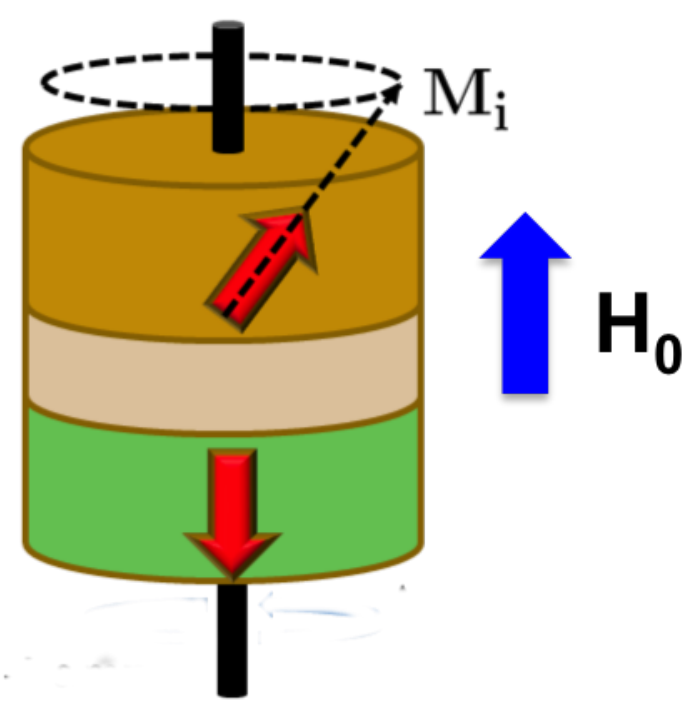

Figure 5.2: Configuration of fixed and free layers of an out-of-plane spin torque nano-oscillator.

The effective magnetic field $\mathbf{H}_{\mathbf{e f f}}$ in the considered case has the simple form:

$$
\mathbf{H}_{\mathrm{eff}}=\left(H_{0}-4 \pi M_{z}\right) \mathbf{e}_{\mathbf{z}}
$$

where $\mathbf{e}_{\mathbf{z}}$ is the unit direction normal to the plane of the free layer, $H_{0}$ is the external bias field, and the term $-4 \pi M_{z}$ describes the influence of the demagnetization field.

In all the practically interesting cases the influence of the conservative torque $\gamma_{0}\left(\mathbf{M} \times \mathbf{H}_{\text {eff }}\right)$ in Eqn. 5.7 is much larger than the influence of the nonconservative damping term and spin-transfer torques. Thus, it is convenient to rewrite Eqn. 5.3 using the normal coordinates of the conservative system, in which the conservative term has the simplest possible form. To get the insight about the possible form of the normal coordinates for the Landau-Lifshitz equation 2.15, one can consider conservative Landau-Lifshitz equation, linearized about the equilibrium orientation of magnetization $\mathbf{M}=M_{0} \mathbf{e}_{\mathbf{z}}$ : 


$$
\begin{aligned}
& \left(\frac{d M_{x}}{d t}\right)_{\mathrm{cons}}=-\omega_{0} M_{y} \\
& \left(\frac{d M_{y}}{d t}\right)_{\mathrm{cons}}=+\omega_{0} M_{x}
\end{aligned}
$$

where $\omega_{0}=\gamma_{0}\left(H-4 \pi M_{0}\right)$ is the ferromagnetic resonance (FMR) frequency of the normally magnetized magnetic film. Note, that the third component of the magnetization vector is constant in the linear approximation $\left(M_{z}=M_{0}\right)$ due to the conservation of the length of the magnetization vector $|M|=M_{0}$. Two real equations for the magnetization projections $M_{x}$ and $M_{y}$ can be rewritten as one complex equation (in the nonlinear regime) for the dimensionless complex variable $c=\left(M_{x}-i M_{y}\right) / \sqrt{2 M_{0}\left(M_{0}+M_{z}\right)}$ :

$$
\left(\frac{d c}{d t}\right)_{\mathrm{cons}}=-i \omega(p) c
$$

where $\omega(p)$ is the nonlinear precession frequency:

$$
\omega(p)=\omega_{0}+2 \omega_{M} p
$$

where $\omega_{M}=4 \pi \gamma_{0} M_{0}$.

One can see that Eqn. 5.10, which describes the nonlinear magnetization precession, has a very simple form and coincides with the conservative part of the universal model. The general solution of Eqn. 5.10 can be easily found in the form:

$$
c(t)=\sqrt{p} \exp \left[-i \omega(p) t+i \phi_{0}\right]
$$


where $p$ and $\phi_{0}$ are, respectively, the dimensionless power and the initial phase of the precession. Such simple description of the nonlinear precession regime is made possible solely by the use of the proper normal coordinates of the systemcomplex spin wave amplitude $c$.

Now, we will consider corrections to the equation of motion caused by the damping and spin-transfer torques. One can obtain the following correction caused by the Gilbert $(\alpha)$ damping torque:

$$
\left(\frac{d c}{d t}\right)_{\text {damp }}=-\Gamma_{+}(p) c
$$

where

$$
\Gamma_{+}(p)=\Gamma_{G}\left(1+Q p+Q^{\prime} p^{2}\right)
$$

and $\Gamma_{G}=\alpha \omega_{0}, Q=2 \omega_{0} / \omega_{M}-1$ and $Q^{\prime}=-2 \omega_{M} / \omega_{0}$. In all physically-relevant range of complex amplitudes $c$ the damping function $\Gamma_{+}(p)$ is positive and Eqn. 5.13 leads to the reduction of spin wave amplitude $c$ with time. For sufficiently small spin wave amplitudes, when one can neglect nonlinear terms in Eqn. 5.14, this reduction is exponential with the linear rate $\Gamma_{G}$, which represents the half-linewidth of the linear ferromagnetic resonance.

It is important to note, that the damping function $\Gamma_{+}(p)$ is nonlinear, even if one uses the usual linear form of the Gilbert damping torque $(\alpha)$. Thus, the damping rate of spin wave excitations depends on the spin wave power $p$ and, generally, increases with $p$. The use of the nonlinear form of the damping torque will lead to the same functional form of the damping correction as in Eqn. 5.13, but with different values of nonlinear damping coefficients $Q$ and $Q$. This correction is qualitatively important in the case of an in-plane magnetized free layer.

In a similar way one can find corrections to the dynamical equation for the spin wave amplitude $c$ caused by the spin-transfer torque. In the case when the bias current is spin- 
polarized along $\mathbf{e}_{\mathbf{z}}$ direction, this correction has the form:

$$
\left(\frac{d c}{d t}\right)_{\operatorname{spin}}=+\Gamma_{-}(p) c
$$

Here, $\Gamma_{-}(p)$ is given by:

$$
\Gamma_{-}(p)=\sigma_{0} I(1-p)
$$

where $\sigma_{0}=\eta \mu_{B} / e V$. For $I>0, \Gamma_{-}(p)$ is positive in physically relevant range of complex spin wave amplitudes $c(p<1)$, and Eqn. 5.15 leads to the increase of the amplitude $c$ with time, i.e., spin-transfer torque creates effective negative damping for the magnetization precession in the free magnetic layer.

Collecting together contributions from the all three torques, we get:

$$
\left(\frac{d c}{d t}\right)=\left(\frac{d c}{d t}\right)_{\mathrm{cons}}+\left(\frac{d c}{d t}\right)_{\mathrm{damp}}+\left(\frac{d c}{d t}\right)_{\mathrm{spin}}
$$

and the dynamical equation for the spin wave amplitude $c$ takes the form:

$$
\frac{d c}{d t}+i \omega(p) c+\Gamma_{+}(p) c-\Gamma_{-}(p) c=0
$$

which exactly coincides with the universal oscillator model without the external driving force $f(t)$. Thus, the dynamics of the considered here simplest spin-torque oscillator can be exactly described by the universal model with properly defined nonlinear frequency $\omega(p)$, natural positive damping $\Gamma_{+}(p)$, and effective negative current-induced damping $\Gamma_{-}$. 


\subsubsection{Precession Generation}

To find the stationary solution of the free self-oscillator equation (Eqn. 5.18), we rewrite it as a system of two real equations for the power $p=|c|^{2}$ and phase $\phi=\arg (c)$ of the oscillations:

$$
\begin{aligned}
\frac{d p}{d t} & =-2\left[\Gamma_{+}(p)-\Gamma_{-}(p)\right] p \\
\frac{d \phi}{d t} & =-\omega(p)
\end{aligned}
$$

Here, the first equation determines the dynamics of the oscillation power $p$ in an autonomous self-oscillator, whereas the second one describes the dynamics of the oscillation phase $\phi$ or defines the generated frequency $d \phi / d t$.

According to Eqn. 5.19, there are two possible types of stationary $(d p / d t=0)$ solutions. The first stationary solution $p=0$ is trivial and corresponds to the absence of any oscillations. Linearizing the equation near this zero-power solution $(p \rightarrow 0)$, one obtains the equation:

$$
\frac{d p}{d t}=-2\left[\Gamma_{+}(0)-\Gamma_{-}(0)\right] p
$$

It is clear from the linearized equation, that the zero-power solution is stable and, therefore, physically realizable, when $\Gamma_{+}(0)>\Gamma_{-}(0)$. Thus, the condition $\Gamma_{+}(0)=\Gamma_{-}(0)$ determines the threshold of appearance of an oscillation having a nonzero power, or, in other words, the threshold of generation in an self-oscillator.

Using Eqns. 5.14 and 5.16 for defining the parameters of the universal oscillator model of an STNO, one obtains the expression for the threshold current $I_{t h}$ of microwave generation 
in the case of a spin-torque nano-oscillator:

$$
I_{t h}=\frac{\Gamma_{G}}{\sigma}
$$

This equation implicitly determines the dependence of the threshold current on the external bias field, geometrical sizes, and other parameters of the STNO.

The second type of stationary solutions of Eqn: 5.19 is a nonzero solution $p=p_{0}$, where $p_{0}$ is determined from the condition $\Gamma_{+}\left(p_{0}\right)=\Gamma_{-}\left(p_{0}\right)$. This equation has a clear physical meaning: in the stationary regime $\left(p=p_{0}\right)$, energy losses due to the natural energy dissipation $\left(\Gamma_{+}\left(p_{0}\right)\right)$ should be exactly compensated by the energy supply from the external energy source $\left(\Gamma_{-}\left(p_{0}\right)\right)$, and the total effective damping $\left(\Gamma_{+}\left(p_{0}\right)-\Gamma_{-}\left(p_{0}\right)\right)$ should be exactly equal to zero.

Using Eqn. 5.19, the stationary power $p_{0}$ for the spin-torque nano-oscillator can be written as:

$$
p_{0}=\frac{I / I_{t h}-1}{I / I_{t h}+Q}
$$

The stationary power (Eqn. 5.23) equals to zero at the threshold of generation (i.e., $\left.I=I_{t h}\right)$ and gradually increases with the increase of the ratio $I / I_{t h}$ up to a limiting value of $p_{\infty}=1$ for $I / I_{t h} \rightarrow \infty$. The dependence of the stationary power $p_{0}$ on the current ratio for several different nonlinear damping coefficients $Q$ is shown on Fig. 5.3.

Similarly, the frequency of generation is one of the most important parameters of an self-oscillator. Equation 5.20 states that the instantaneous oscillator frequency $d \phi / d t$ is, at each moment of time, equal to the nonlinear frequency $\omega(p(t))$ determined by the current value of the oscillation power $p(t)$. Thus, the frequency $\omega_{g}$, generated in the stationary 


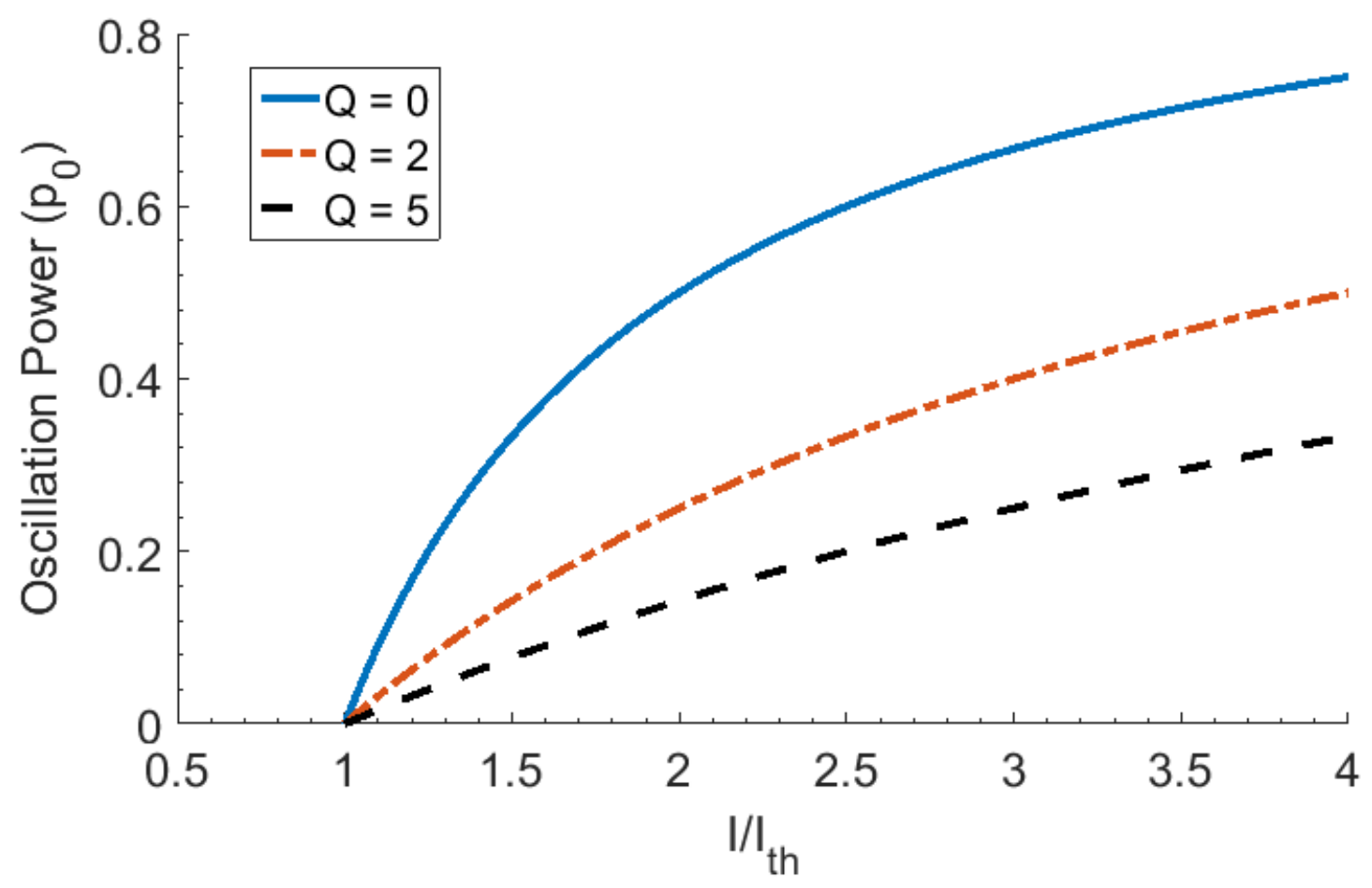

Figure 5.3: Dependence of the stationary power $p_{0}$ of a spin-torque nano-oscillator on the current ratio $I / I_{t h}$ for several values of the nonlinear damping parameter $Q$.

oscillation regime $\left(p=p_{0}=\right.$ const $)$, is given by the equation $\omega=\omega_{g}\left(p_{0}\right)$, which corresponds to the linear time dependence of the oscillation phase:

$$
\phi(t)=-\omega_{g} t+\phi_{0}=-\omega\left(p_{0}\right) t+\phi_{0}
$$

where $\phi_{0}$ is an arbitrary initial phase of the oscillations. Then, the full stationary solution $c_{0}(t)$ for the complex amplitude $c$ of the self-oscillation can be written as:

$$
c_{0}(t)=\sqrt{p_{0}} \exp \left[-i \omega_{g} t+i \phi_{0}\right]
$$




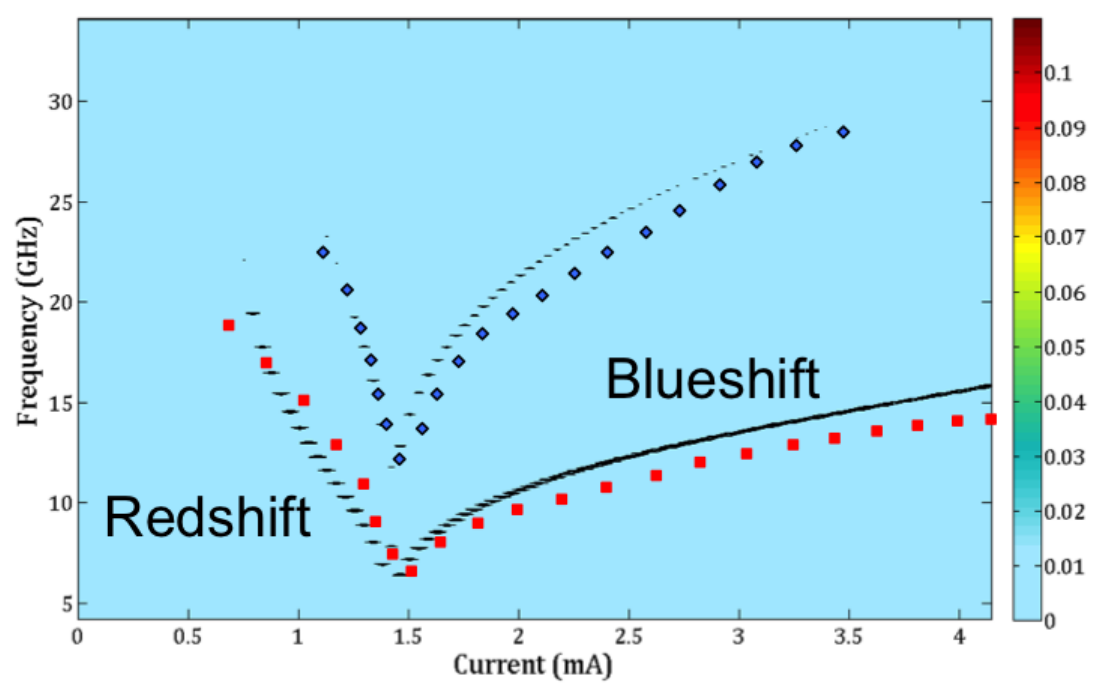

Figure 5.4: Frequency versus applied current behavior in STNOs. The lines represent the Verilog-A model predictions, while the red and blue points represent experimental results [50].

Using Eqn.5.23 and 5.11, one can obtain an explicit expression for the frequency, generated by a spin-torque oscillator:

$$
\omega_{g}=\omega_{0}+N \frac{I / I_{t h}-1}{I / I_{t h}+Q}
$$

The dependence is shown in Fig. 5.4 for positive (blue frequency shift) and negative (red frequency shift) values of $N$. One can, also, find the frequency tunability $d \omega_{g} / d \mu$ with respect to the variation of some control parameter $\mu$ of the oscillator:

$$
\frac{d \omega_{g}}{d \mu}=\frac{d \omega}{d \mu}+N \frac{d p_{0}}{d \mu}
$$

where $N=d \omega(p) / d p$ is the nonlinear and all the derivatives are taken at the stationary operation point $p=p_{0}$. The first term accounts for the direct dependence of the oscillator frequency $\omega(p)$ on the control parameter $\mu$, while the second term describes the indirect 


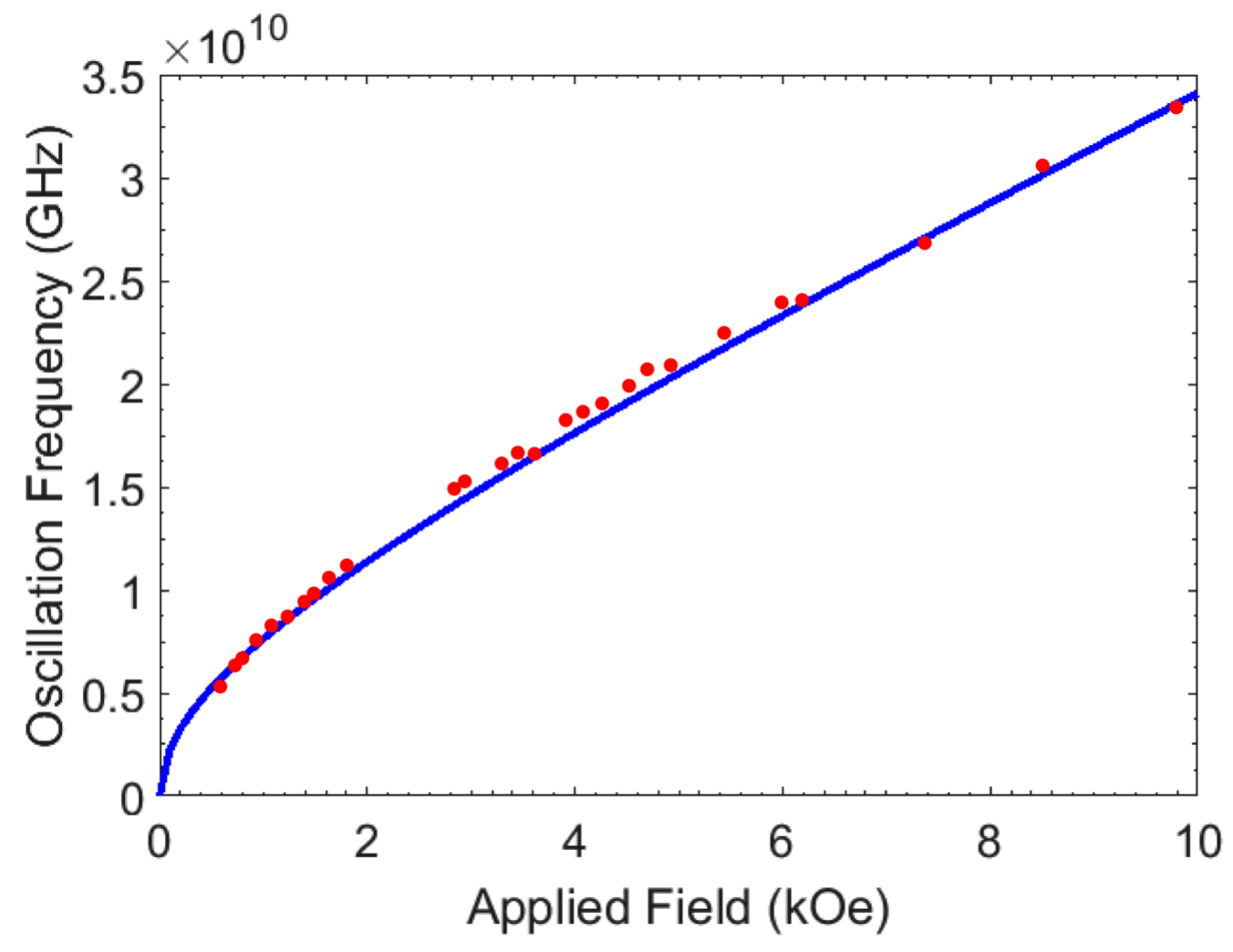

Figure 5.5: Frequency of current-induced microwave oscillations $\omega_{g}$ in the in-plane magnetized nano-contact as a function of the bias magnetic field $H_{0}$. Solid circles - experimental data from [74]; Solid line-Verilog model.

changes in the generation frequency $\omega\left(p_{0}\right)$ due to the dependence of the stationary power $p_{0}$ and on the parameter $\mu$.

\subsubsection{Model Validation}

The Verilog-A based model gave a natural explanation of a number of qualitative features of microwave generation in spin-torque nano-oscillators. First of all, it explained the dependencies of the generation frequency $\omega_{g}$ and frequency-current slope $d \omega_{g} / d I$ on the direction and magnitude of the external bias field, allowed one to estimate the threshold current $I_{t h}$ of microwave generation, etc. Here we present several examples of experimental data that can be quantitatively explained by the simple nonlinear oscillator model (Eqn. 5.19). In Fig. 5.5 


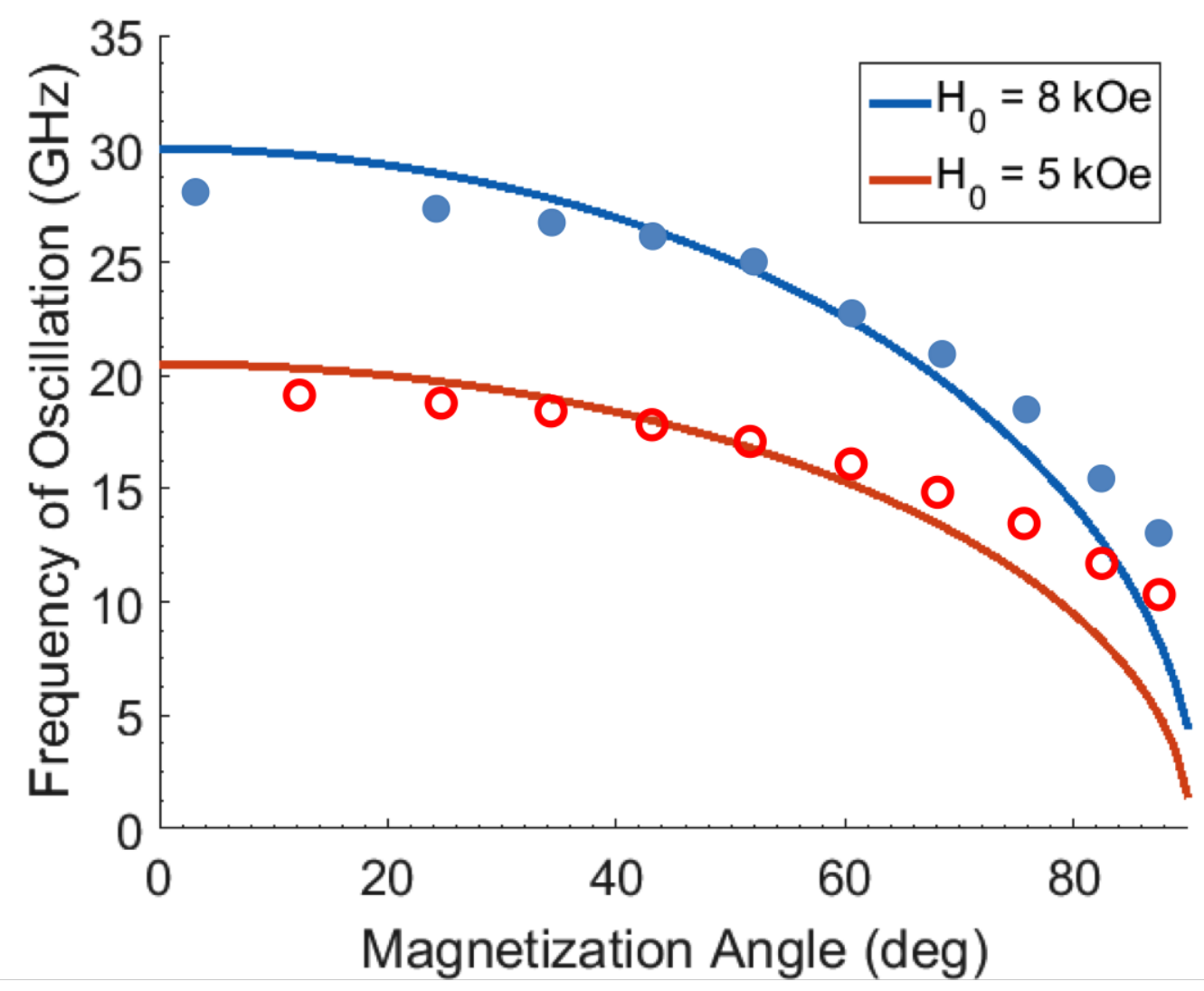

Figure 5.6: Frequency of current-induced microwave oscillations $\omega_{g}$ as a function of the outof-plane magnetization angle $\theta_{0}$ for $H_{0}=8 \mathrm{kOe}$ (solid circles) and $H_{0}=5 \mathrm{kOe}$ (open circles). The solid and open circles represent the experimental data from [73]; solid lines-Verilog model.

shows the dependence of the generation frequency $\omega_{g}$ on the bias magnetic field $H_{0}$ for an inplane magnetized magnetic nano-contact. One can see, that the nonlinear oscillator model of current-induced oscillations can give satisfactory quantitative explanation of the experimental data in all the range of bias magnetic fields $H_{0}$. While the shape of the dependence of the generation frequency $\omega_{g}$ on $H_{0}$ is qualitatively similar to the dependence of the FMR frequency $\omega_{0}$ on the bias field $H_{0}$, the quantitative values of $\omega_{g}$ are significantly below $\omega_{0}$. This is explained by the fact that the experiments [74] were performed significantly above the threshold of generation (where the current ratio parameter was equal to $I / I_{t h}=1.56[79]$ ), 
and, since the nonlinear frequency shift coefficient $N$ is negative for an in-plane magnetized film, the generation frequency $\omega_{g}=\omega\left(p_{0}\right)$ was shifted down from the FMR frequency $\omega_{0}$ (see Eqn. 5.26).

In Fig. 5.6, we shows the dependence of the generation frequency g on the out-of-plane magnetization angle $\theta_{0}$ for two values of the bias magnetic field $H_{0}$. The points represent experimental data from [73], while the lines show Verilog-A model results in the framework of nonlinear oscillator model. Here, again, the simple theory gives a good quantitative description of the experimental data.

\subsection{Electrically Coupled STNOs}

\subsubsection{Magnetic vs. Electrical Synchronization}

While phase locking via magnetic spinwaves has been shown between two spin torque oscillators $[84,74,75]$, it still remains uncertain if this process can be scaled up for fabrication, or even synchronization, when many STNOs are involved [85].

An alternative to magnetic synchronization is to use the magnetoresistance effect to convert the magnetic oscillations in the free layer into an alternating current signal through the MTJ. Then two or more STNOs can be connected electrically to achieve synchronization. Each STNO in an electrically coupled array can operate independently and does not require specialized features (e.g. shared free layer) to achieve synchronization. This allows for a variety of circuit topologies which would be difficult to realize in a purely magnetic system. In spinwave-coupled STNOs, the geometric layout of the oscillators will determine functionality, 


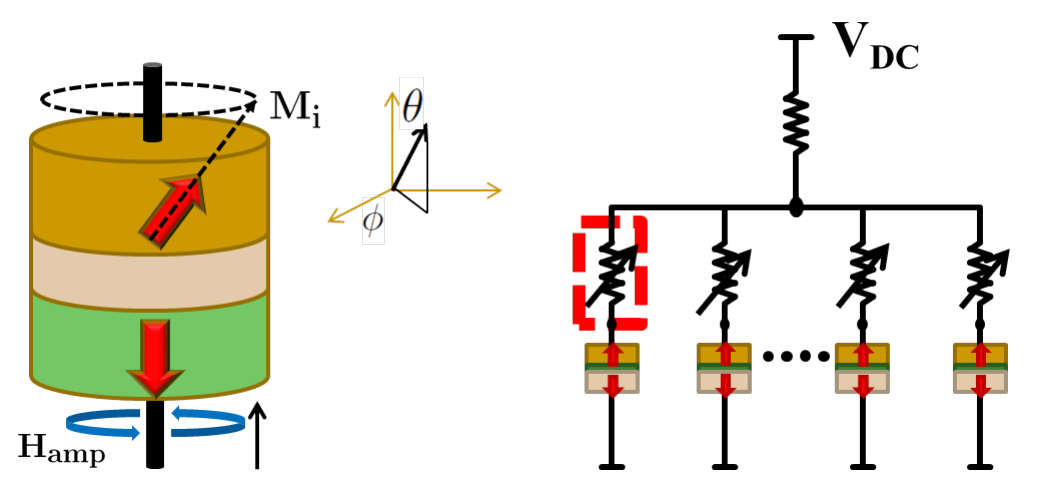

Figure 5.7: (a) The relative orientation of the magnetization vector and the effective magnetic field which synchronizes the STNOs. (b) A general layout of an electrically-coupled parallelconnected STNO array. The variable resistor in the schematic represents the ability to change the current through each parallel branch of the array to set the $\mathrm{DC}$ current $\left(I_{D C, i}\right)$. This produces an oscillating signal $\left(I_{A C, i}\right)$ which combines on the common node with the signals from the other parallel branches.

whereas in electrically-coupled arrays the oscillator connectivity is determined by the more flexible circuit topology. Furthermore, the electrical nature of the signals can be used to integrate the STNOs with MOSFET transistors and other conventional circuit elements. This feature makes a hybrid MOSFET/STNO circuit attractive for computational purposes.

\subsubsection{Synchronization Dynamics}

One possible method of increasing the power of spin torque nano-oscillators has been to synchronize them to a common frequency $[75,86,87,79]$. Electrically synchronizing multiple STNOs has been previously considered for both series and parallel configurations [88, 89, 90,

91]. We have shown previously $[92,93,94]$ that in a parallel array of STNOs with out-of-plane fixed and free layers, oscillators with similar frequencies can exchange energies through their AC signal, slowing down or speeding up their oscillations until they achieve synchronization. We now examine the synchronization dynamics of the circuit shown in Fig. 5.7. Each parallel STNO will have a DC current, $I_{d c, i}$, which determines the initial frequency of its operation. 
This in turn will generate an AC current, $I_{a c, i}$, due to the TMR of the magnetic junction. By Kirchoff Current Law, the common node will have a combined AC current of $I_{a c}=\Sigma_{i} I_{a c, i}$. This AC current will re-distribute the energy throughout the array. To quantify the energy gain/loss in one of the STNO, we look at the $\mathbf{H}_{\text {amp }}$ component of the effective magnetic field in the LLG equation (Eqn. 5.3). In this case, $\mathbf{H}_{\mathbf{a m p}}=\xi I_{a c} \phi_{\mathbf{a c}}$, where $\xi$ is a material dependent property and $\phi_{\mathbf{a c}}$ is the angular unit vector in-plane to the free layer of the STNO. Therefore, for the $i$-th STNO, the energy injected/removed by the magnetic field $\mathbf{H}_{\mathbf{a m p}}$ is given by:

$$
\begin{aligned}
E_{i} & =-\mu_{0} M_{s} V \int \mathbf{H}_{\mathbf{a m p}} \cdot d \mathbf{M}_{\mathbf{i}} \\
& =-\mu_{0} M_{s} V \xi \int \sum_{j} I_{a c, j} \phi_{\mathbf{a c}, \mathbf{j}} \cdot d \mathbf{M}_{\mathbf{i}} \\
& =-\sum_{j} \vartheta_{j} m_{i} \cos \left(\phi_{i}-\phi_{j}\right)
\end{aligned}
$$

where $\vartheta_{j}=\pi \mu_{0} M_{s} V K I_{a c, j}$, and $m_{i}=\sqrt{\frac{1}{2}\left[m_{x}^{2}+m_{y}^{2}+\left(m_{x}^{2}-m_{y}^{2}\right) \sin \left(2 \phi_{a c, j}\right)\right]}$ represents the projection of $\mathbf{M}_{\mathbf{i}}$ onto $\phi_{\mathbf{a c}, \mathbf{j}}$. Finally, $\phi_{i}$ and $\phi_{j}$ are phase components of the $i$-th and $j$-th oscillator respectively.

Now that we have an expression for the energy landscape of STNOs, we can determine how the frequency of oscillation changes to respond to the change in energy. First, we recognize that the phase is related to its frequency as $\phi_{i}=\phi_{i, 0}+2 \pi f_{i} t$. Therefore, the changes in the 
phase can be represented as:

$$
\begin{aligned}
\frac{d \phi_{i}}{d t} & =2 \pi f_{i}+\delta f_{i} \\
& =2 \pi f_{i}+\left(\frac{d f_{i}}{d E_{i}}\right) E_{i} \\
& =2 \pi f_{i}-\sum_{j} \Delta_{i j} \cos \left(\phi_{i}-\phi_{j}\right)
\end{aligned}
$$

where $\phi_{i}$ and $f_{i}$ are respectively the phase and frequency of the oscillator, and the coupling

constant $\Delta_{i j}=-\left(\frac{d f_{i}}{d E_{i}}\right) \vartheta_{j} m_{i}$ is between the two oscillators which depends on the material properties of the device and relative frequency distance. Equation (5.29) represents the exact dynamics outlined by Kuramoto's model of weakly coupled oscillators [95]. Kuramoto's oscillation model is a general principle which applies to many emergent behaviour phenomena in nature $[96,97,98,99]$. An important application was first proposed by Hoppensteadt and Izhikevich [100] to model associative memory in neural networks. In the next chapter, we will consider the similarities between the Hoppensteadt model and an array of parallel connected STNOs.

\subsection{STNO Geometry \& Oscillations}

In the previous sections, we have considered the properties of a general parallel connected STNO array. However, to create a physical array there are several device-level considerations that must be taken into account. There are many different geometric configurations of the fixed and free layers of a spin torque oscillator. Each configuration can generate different modes of oscillation depending on the biasing current and applied external magnetic fields. 

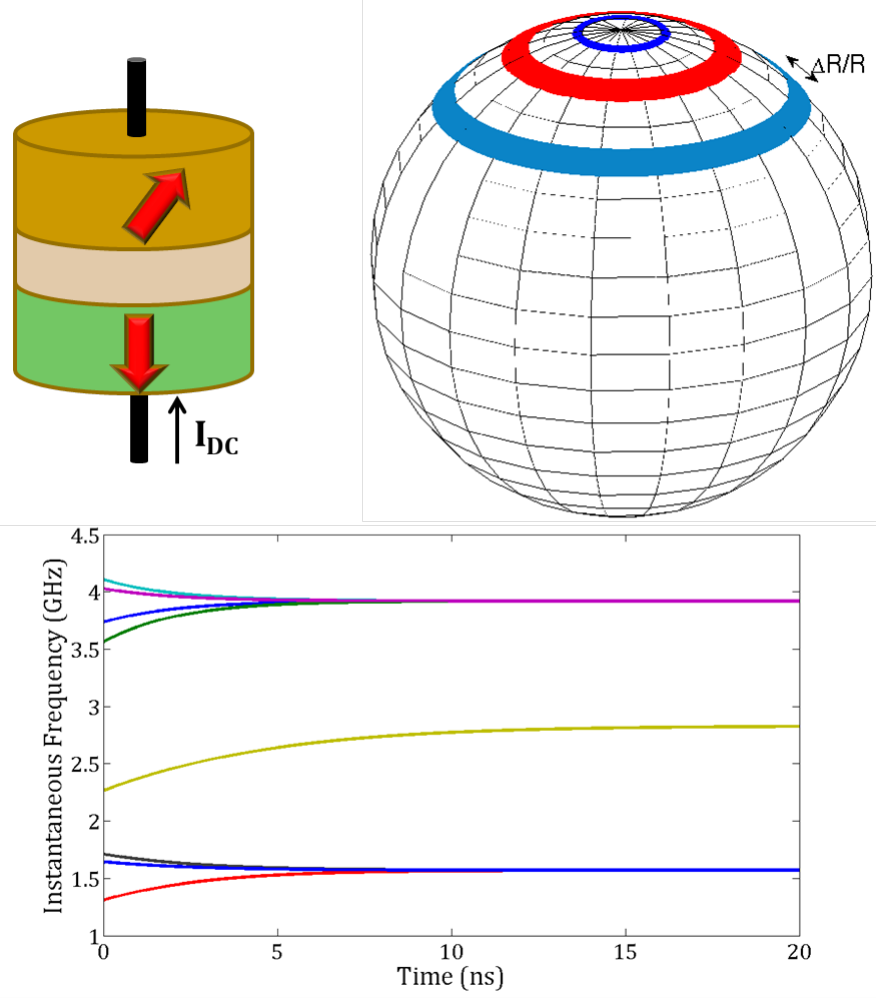

Figure 5.8: (Top) A Perpendicular STNO. This configuration exhibits circular orbits which produce almost harmonic oscillations. However, the amplitude of the electrical signal is weak due to the infinitesimal variation in $\Delta R / R$ when the angle of precession is constant. Here we show multiple orbits corresponding to different DC currents and frequencies. (Bottom) Transient simulation showing changes in frequency and synchronization of almost harmonic STNOs.

First, we consider two configurations-perpendicular (Figure 5.8) and mixed-both of which use perpendicular magnetic anisotropy (PMA) free layers. These geometries were chosen because they can produce oscillations without the need for an external magnetic field [101, 102, 103]; this makes it much easier to operate a large-scale array without the need of a localized magnetic field. The mode of oscillation for these two configurations are different which creates a trade-off between synchronization and signal strength. 

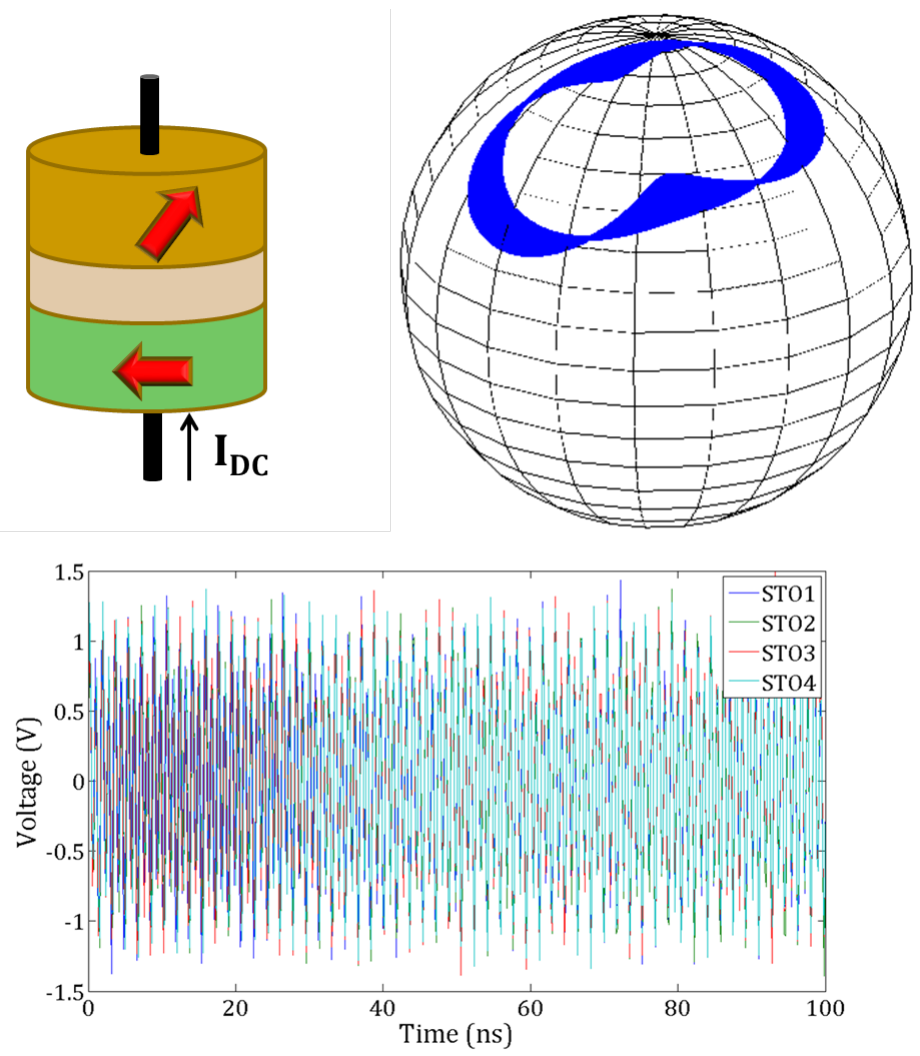

Figure 5.9: Top: Mixed Geometry STNO. The mixed geometry STNO exhibits complex orbital precessions which lead to nonharmonic oscillations. Bottom: Transient analysis shows the complex periodic orbit of four mixed STNOs with similar frequencies. This configuration creates a large electrical signal because of the large variations in magnetoresistance. At the end, all four oscillators are shown to synchronize.

\subsubsection{Harmonicity \& Signal Strength}

When considering these two geometries of STNOs, the oscillation mode plays a significant role in the synchronization behaviour and the amplitude of the electrical signal generated by the MTJ. The amplitude of the electrical signal depends on the magnetoresistance of the MTJ. This effect can be estimated as the angular difference between the free and fixed layer given by the equation,

$$
\frac{\Delta R}{R}=1-P^{2} \cos (\theta)
$$



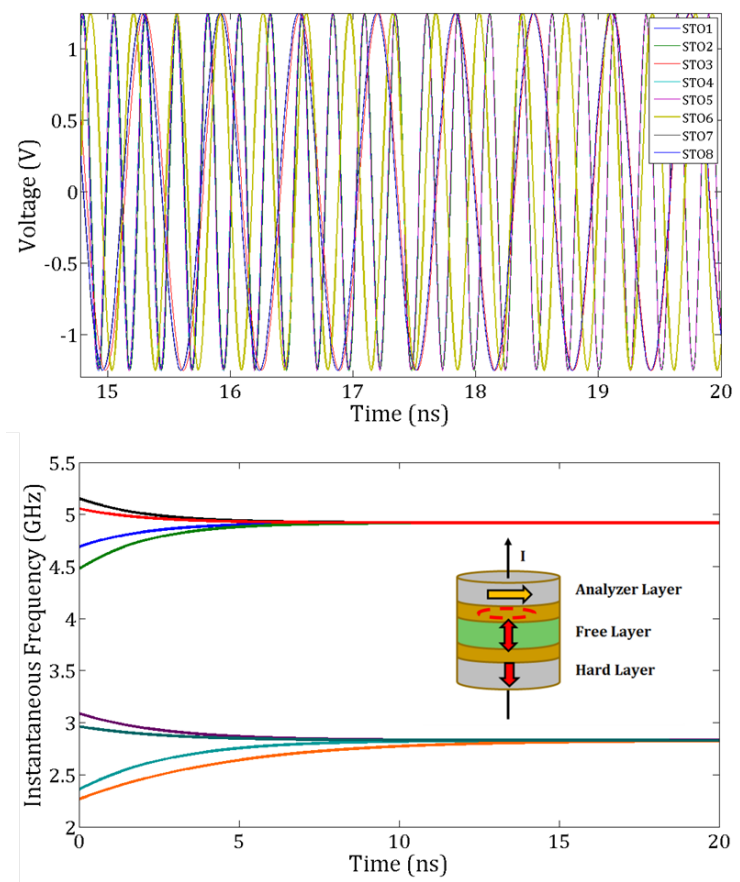

Figure 5.10: Dual Barrier Magnetic Tunnel Junction. (Top) The oscillations in the DMTJ are both harmonic and have large electrical signal. (Bottom) The transient frequency behavior of eight DMTJ STNOs as they synchronize.

where $R$ is the average MTJ resistance, $\Delta R$ is the deviation from the average, $P$ is the effective polarization by the fixed layer and $\theta$ is the angular difference between the free and fixed layer.

In the perpendicular geometry, where both the free and fixed layer are PMA materials, the trajectory of the magnetization is approximately circular, producing a simple almost harmonic oscillation. With almost harmonic oscillations, synchronization between multiple oscillators can be achieved with frequency differences as large as 22\% (Figure 5.8(Bottom)) and within $\sim 100$ periodic cycles. However, because of the circular trajectory, there is very little change in $\Delta R / R$ from Equation (5.30), creating very small electrical signals (Figure 5.8(Top)). This, in turn, prevents effective communications between the oscillators in the array. 

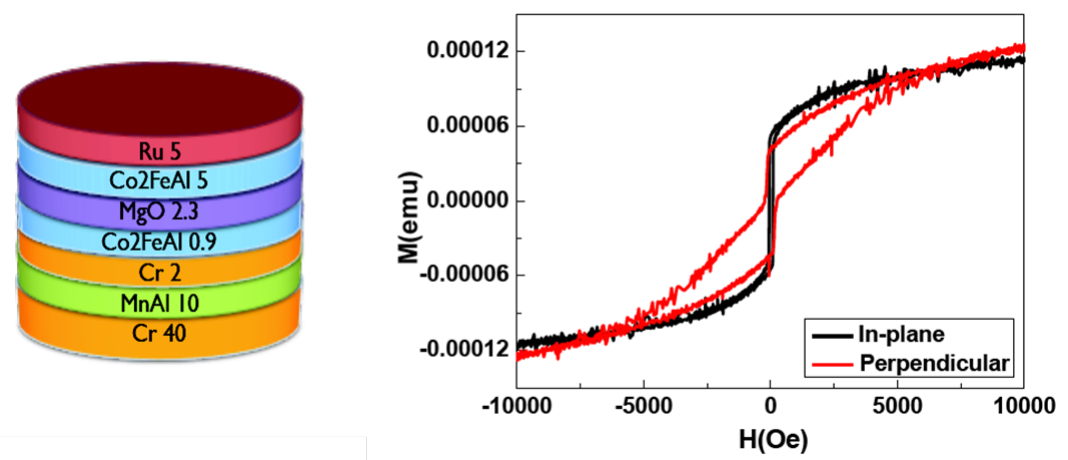

Figure 5.11: (a): Schematic diagram of a perpendicular spin torque nano-oscillator in design. (b): In-plane and out-of-plane hysteresis loops of the multilayer structure.

In a mixed configuration the fixed layer is in-plane while the free layer is out-of-plane (Figure 5.9). In this geometry, the orbit of oscillation produces a Lissajous curve and thus produces a complex periodic signal. This is advantageous from an electric signal point of view due to the large change in $\Delta R / R$. However, simulation results show that synchronization becomes difficult as the frequency bandwidth of coherence is reduced to $2-3 \%$ with much larger coherence time than for harmonic oscillations.

\subsubsection{Dual Barrier MTJ}

In order to take advantage of the positive aspects of both configurations, we propose combining the two geometries in a trilayer structure. This dual barrier magnetic tunnel junction (DMTJ) consists of PMA fixed and free layers and an in-plane analyzer layer. During operation, the PMA fixed layer induces harmonic oscillation in the free layer while the in-plane analyzer layer creates large changes in $\Delta R / R$ yielding a large electrical signal.

It should be noted that DMTJs have been realized experimentally [104] though those devices used only in-plane ferromagnets for all three layers. The fabrication of PMA DMTJs 
is an area of active research (Figure 5.11).

Simulations results show that the DMTJ structure maintains near harmonic oscillations and thus can have synchronization frequency bandwidths as large as $18 \%$ with coherence times on the same order as the perpendicular geometry (Figure 5.10). At the same time, the DMTJ maintains a large electrical signal comparable to the mixed geometry. With this device geometry, an array of STNOs can be created which is robust enough for computation. 


\section{Chapter 6}

\section{Applications of Synchronized Spin}

\section{Torque Nano-Oscillators}

In the previous chapter, we developed an accurate Verilog-A model of the spin torque nanooscillator and showed that in a parallel, electrically-connected array, the spin torque oscillators can mutually synchronize. Furthermore, we showed analytically that such an architecture follows the dynamics outlined by Kuramoto's model of weakly couple oscillators [95]. An important application was first proposed by Hoppensteadt and Izhikevich [100] to model associative memory in neural networks. In this chapter we will explore the applications of synchronized spin torque nano-oscillators.

\subsection{STNO Array as an Oscillatory Neurocomputer}

The Hoppensteadt model uses oscillators to implement a neural network. The Non-Boolean nature of the oscillators can realize neural networks with only $N$-connections as opposed 

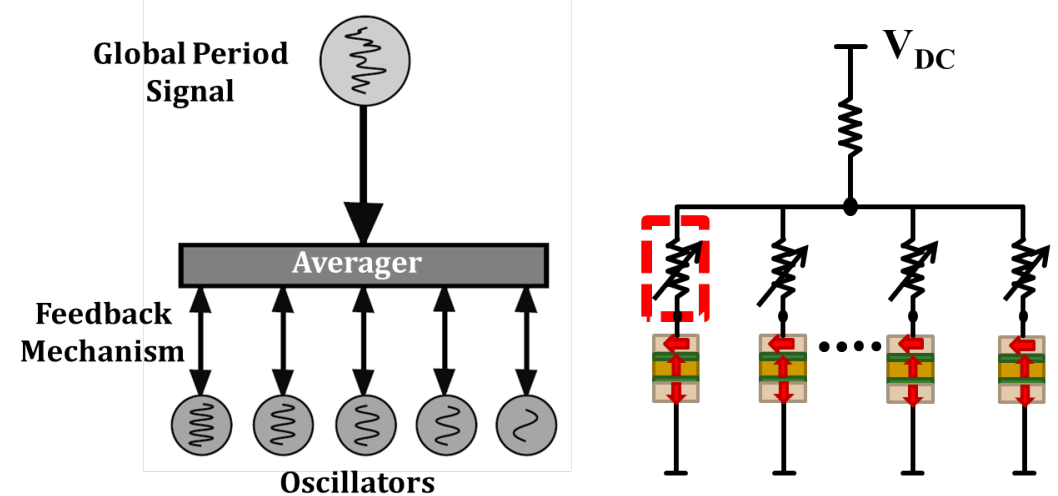

Figure 6.1: STNO array as an oscillatory neurocomputer. (a) Components of an oscillatory neurocomputer (modified from Hoppensteadt et. al). (b) A general layout of an electrically coupled parallel-connected DMTJ-STNO array. The dual barrier magnetic tunnel junction (DMTJ) produces a harmonic signal with a strong voltage signal.

to $N^{2}$-connections required by traditional cross-weighted architectures [78]. Because both systems are manifestations of Kuramoto's oscillation model, the STNO array can act as a physical implementation of Hoppensteadt's oscillatory neurocomputer.

Figure 6.1 shows the similarities between the oscillatory neurocomputer and the STNO array. The STNO array consists of parallel connected devices with the oscillation frequency set by the current running through each branch. The oscillating signals from each parallel branch are summed up in an analogous fashion to the "averager" in the Hoppensteadt scheme and act as a global periodic signal through which the oscillators communicate. The feedback layer is emulated in the STNOs through the magnetization dynamics of the free layer. The overall effect is a change in frequency and phase of the oscillator shown in Eqn. 5.29. When the oscillator frequencies are close they begin to synchronize. In the next section we discuss how such an array can be physically implemented. 

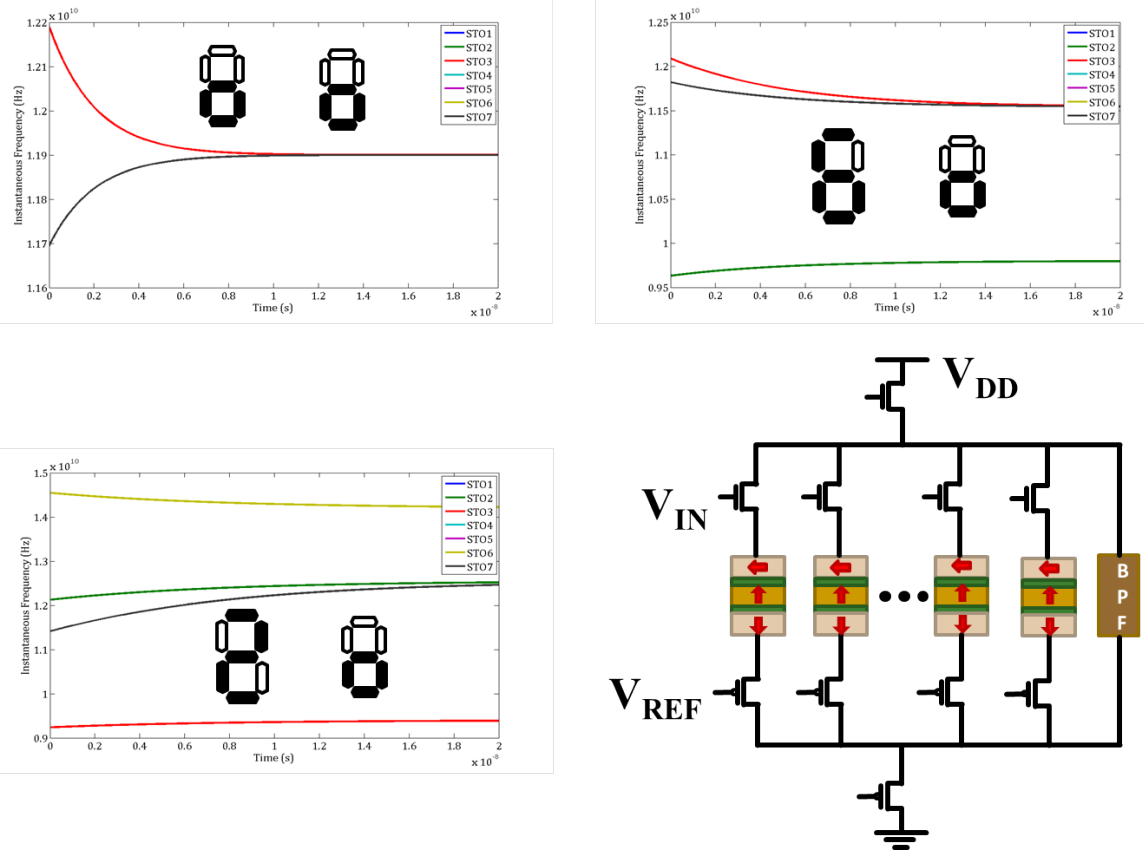

Figure 6.2: Hybrid MOSFET/STNO Array for Pattern Recognition. (Bottom Right Corner) The hybrid circuit consists of DMTJ spin torque oscillators sandwiched by NMOS and PMOS source followers. The $V_{R E F}$ transistors receive the reference vector, while the $V_{I N}$ handle the input vector. The bandpass filter $(\mathrm{BPF})$ is used to isolate frequencies representing matches. (Left Column) Transient analysis showing the synchronization of STNOs over time.

\subsection{Pattern Recognition}

As a first step to an associative memory circuit, we designed a hybrid MOSFET/STNO array which can compare a test vector with a reference vector and give an indication of the degree of match (DOM) between the two data sets. As explained in the previous chapter, we use DMTJ spin torque nano-oscillators for the array.

The MOSFET transistors are used to control the frequency of oscillation by modulating the current in each branch depending on the test vectors. Each of the transistors act as source followers thereby maintaining the same voltage across the DMTJ if both inputs to the transistors are shifted by an equal amount. This ensures that the frequency of the STNO remains unchanged if the reference and test bits are the same. In the test case shown in Fig. 
6.2, an STNO is assigned to each bit of a 7-segment array. The Reference column shows how the reference transistors are assigned-with '0' representing a blank and '1' representing a filled segment. These bits represent patterns which have already been "memorized" by the array. Similarly, the Input column represents the bits for the test pattern which will be compared to the memorized patterns.

In the topmost case, we have a test pattern which is an exact match to one of the memorized patterns. The STNO arrays start at two frequencies representing the bits which are both '0's and both '1's. These frequencies are close enough such that they synchronize together until all oscillators operate at the same frequency. The time for all the STNOs to cohere together was approximately $10 \mathrm{~ns}$ for oscillators operating at $12 \mathrm{GHz}$.

The middle and bottom cases illustrate the scenario where the test pattern is not an exact match to the memorized pattern. As the transient analysis shows, there are additional frequencies that appear when there are mismatches. These frequencies are different enough that they do not synchronize completely with the STNOs which represent matches, though their frequencies are affected. In case of mismatches, the oscillators take longer to reach synchronization, sometimes taking more than twice longer than for a perfect match scenario. If we perform a frequency analysis on the parallel node of the STNO array after all the signals have synchronized, we can get an estimate of the DOM between the test and reference vectors. As the number of oscillator synchronize to a single frequency, the output signal power is boosted. Therefore, if we analyze the signal power near frequencies representing matches, we can determine which memorized pattern represents the best match to the test vector. A bandpass filter can be used to eliminate all frequencies except for the ones representing matches (Figure 6.2). 


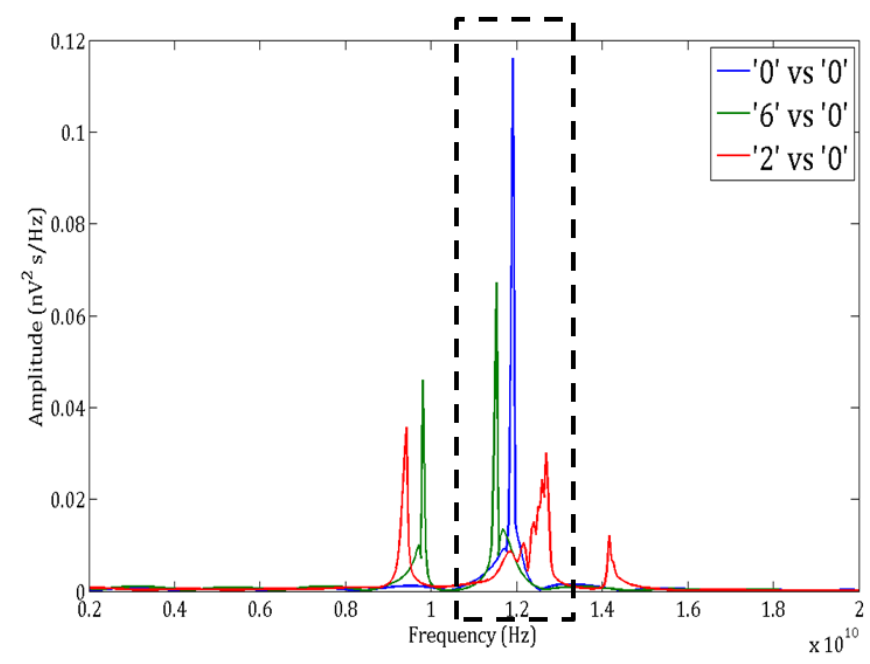

Figure 6.3: The Fourier analysis of the parallel node can be translated as the degree of match. Hence ' 6 ' is a better match than '2'. The dashed box shows the frequencies of the BPF.

This array represents a simple hybrid MOSFET/STNO circuit which can be used to analyze patterns and determine a degree of match. While this toy model is a trivial example of pattern recognition, it displays some of the techniques that can be adapted for more complicated applications. In the next section, we modify the array architecture for texture segmentation.

\subsection{Texture Segmentation}

To humans, an image is not just a random collection of pixels; it is a meaningful arrangement of regions and objects. We can agree about the different regions in the images and recognize the different objects. It has been identified that several factors lead to human perceptual grouping: similarity, proximity, continuity, symmetry, parallelism, closure and familiarity. In computer vision, these factors have been used as guidelines for many grouping algorithms [105]. 


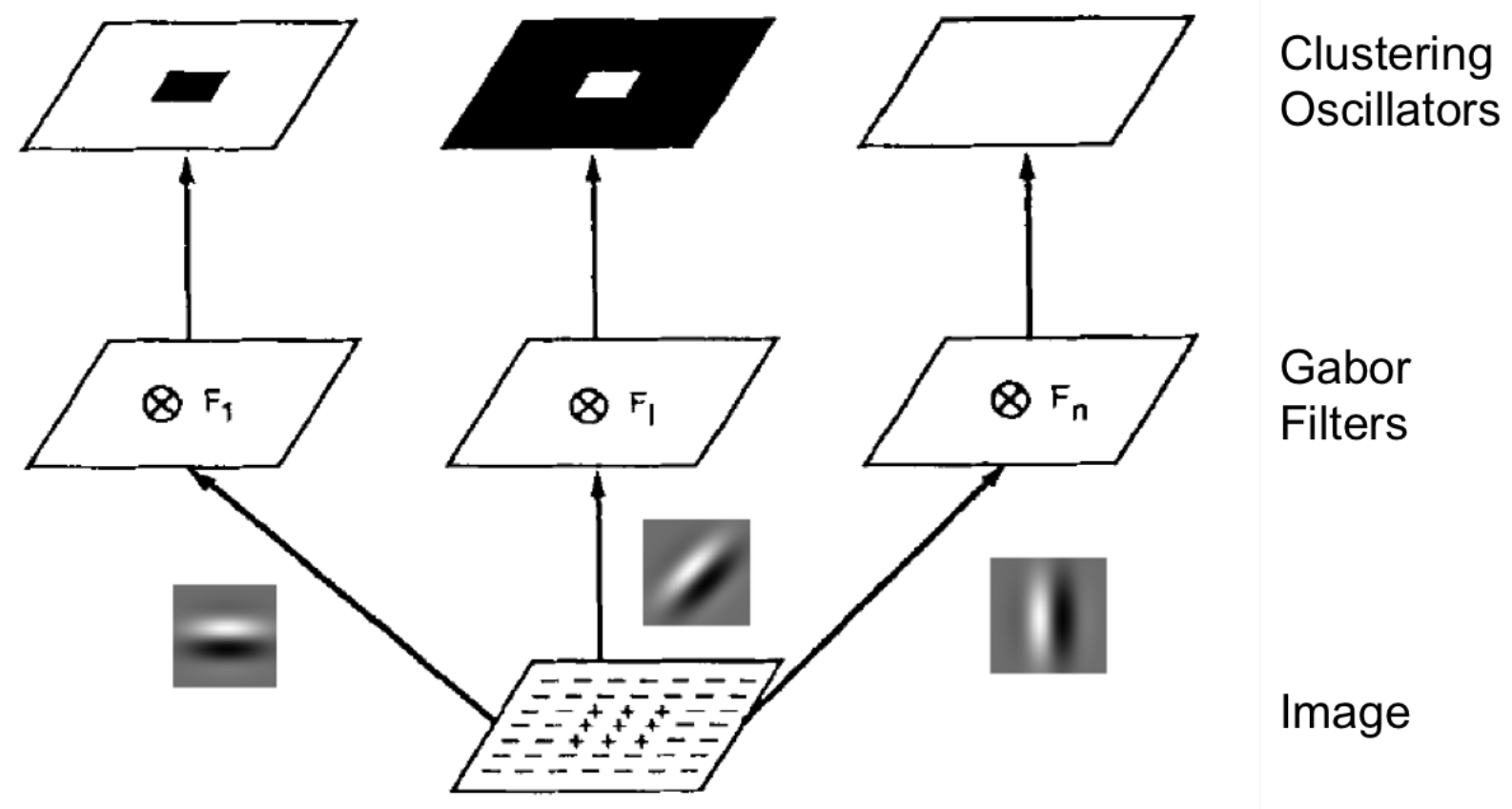

Figure 6.4: Schematic of texture detection using coupled oscillators. The textured image is first filtered through a bank of Gabor filters and the result is fed into an array of oscillators which clusters the spatial data.

The most studied version of grouping in computer vision is image segmentation. Image segmentation techniques can be classified into two broad families (1) region-based, and (2) contour-based approaches. Region-based approaches try to find partitions of the image pixels into sets corresponding to coherent image properties such as brightness, color and texture. Contour-based approaches usually start with a first stage of edge detection, followed by a linking process that seeks to exploit curvilinear continuity. In this section, we will exploit the parallel array architecture of STNOs to implement a region-based segmentation to distinguish different textures within an image.

One of the most useful methods of extracting textures has been filtering methods [81]. In fact, one well known class of filters that are known to achieve both spatial and spatialfrequency localization needed for texture detection is the Gabor function. The process of 


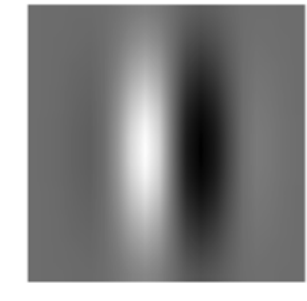

(a)

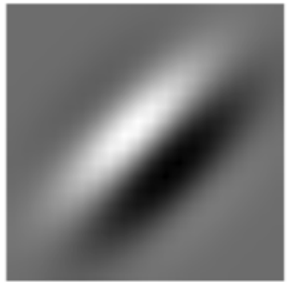

(b)

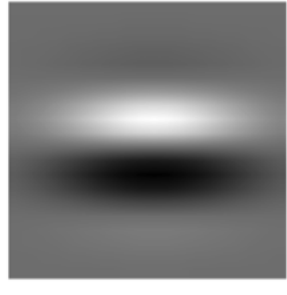

(c)

Figure 6.5: Gabor filters with orientations of (a): $0^{\circ},(\mathbf{b}): 45^{\circ},(\mathbf{c}): 90^{\circ}$

texture segmentation using Gabor filters involves proper design of a filter bank tuned to different spatial-frequencies and orientations to cover the spatial-frequency space; decomposing the image into a number of filtered images; extraction of features from the filtered images; and the clustering of pixels in the feature space to produce the segmented image (Fig. 6.4).

\subsubsection{Gabor Filters}

Gabor filters have the ability to perform multi-resolution decomposition due to its localization both in spatial and spatial-frequency domain. Texture segmentation requires simultaneous measurements in both the spatial and the spatial-frequency domains. Filters with smaller bandwidths in the spatial-frequency domain are more desirable because they allow us to make finer distinctions among different textures. On the other hand, accurate localization of texture boundaries requires filters that are localized in the spatial domain. However, normally the effective width of a filter in the spatial domain and its bandwidth in the spatial-frequency domain are inversely related according the uncertainty principle [106]. That is why Gabor filters are well suited for this kind of problem.

A Gabor function in the spatial domain is a sinusoidal modulated Gaussian. For a 2-D Gaussian curve with a spread of $\sigma_{x}$ and $\sigma_{y}$ in the $x$ and $y$ directions, respectively, and a 
modulating frequency of $u_{0}$, the real impulse response of the filter is given by:

$$
G(x, y)=\frac{1}{2 \pi \sigma_{x} \sigma_{y}} \exp \left\{-\frac{1}{2}\left[\frac{x^{2}}{\sigma_{x}^{2}}+\frac{y^{2}}{\sigma_{y}^{2}}\right]\right\} \cdot \cos \left(2 \pi u_{0} x\right)
$$

The above equations show only an orientation of zero degrees with respect to the x-axis. An arbitrary rotation of the filter can be achieved spatially by rotating the spatial function in the spatial domain in the $x-y$ plane. The frequency $u_{0}$ and the rotation angle $\theta$ define the center location of the filter. By tuning $u_{0}$ and $\theta$ to different center locations we can create multiple filters that cover the spatial-frequency domain. The rotations can be achieved through the transformations:

$$
\left\{\begin{array}{l}
x^{\prime}=x \cos \theta+y \sin \theta \\
y^{\prime}=-x \sin \theta+y \cos \theta
\end{array}\right.
$$

Several orientations of the Gabor filter are shown in Fig. 6.5.

\subsubsection{Oscillator-based Filtering}

The filter process by a Gabor filter is achieved by a convolution operation with the image. Therefore, the filtered image can be represented as [78]:

$$
(F \cdot G)\left(x^{\prime}, y^{\prime}\right)=\int F\left(x^{\prime}-x, y^{\prime}-y\right) G(x, y) d x d y
$$



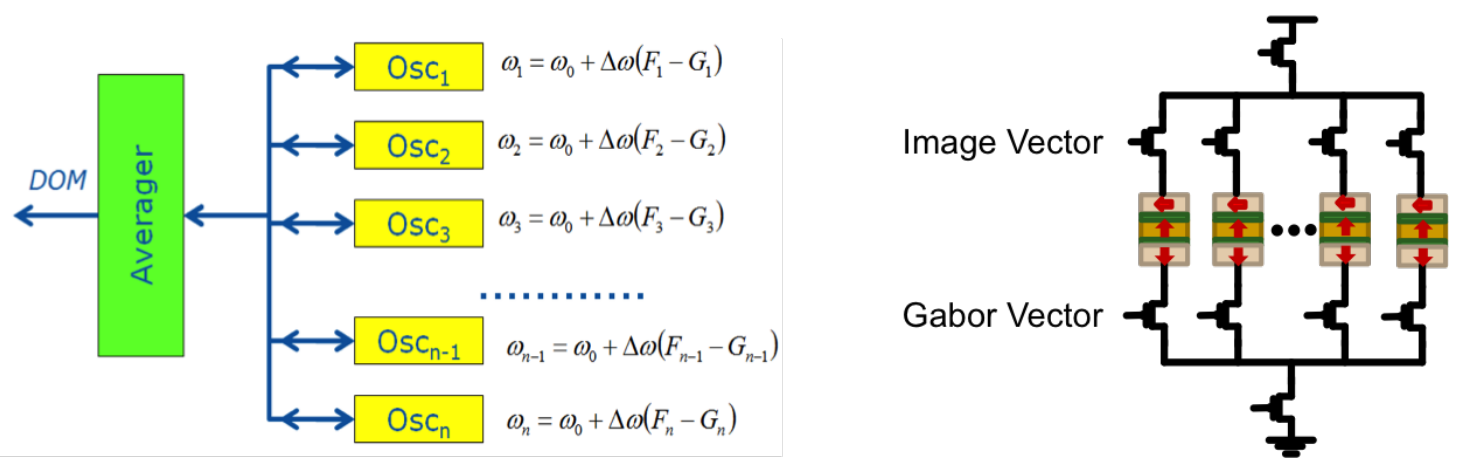

Figure 6.6: Gabor filtering through coupled oscillators using frequency shift keying. The corresponding Gabor filter circuit using a parallel array of STNOs.

where $F$ is the image and $G$ is the Gabor filter function. For 2D pixeled images, this relation can be expressed as a matrix multiplication:

$$
(F \cdot G)_{k l}=\sum_{i, j}\left(F_{k-i, l-j}\right)\left(G_{i j}\right)
$$

It has been shown [77] that the matrix multiplication in Eqn. 6.3 has a direct relationship with the pixel-wise difference between the image and Gabor filter pixels. This suggests that we can approximate the convolution integral (Eqn. 6.2) by using a circuit very similar to the toy model developed in the previous section, since that circuit essentially computed the pixel-wise difference between two vectors.

Furthermore, Nikonov et. al. [107] introduced a coupled oscillator model for implementing the Gabor filter. Nikonov introduced a frequency shift keying (FSK) method for assigning the frequency of oscillation for each of the oscillators. The oscillators have their initial frequencies shifted from the center frequency $\omega_{0}$ by the DC control voltage inputs proportional to the 

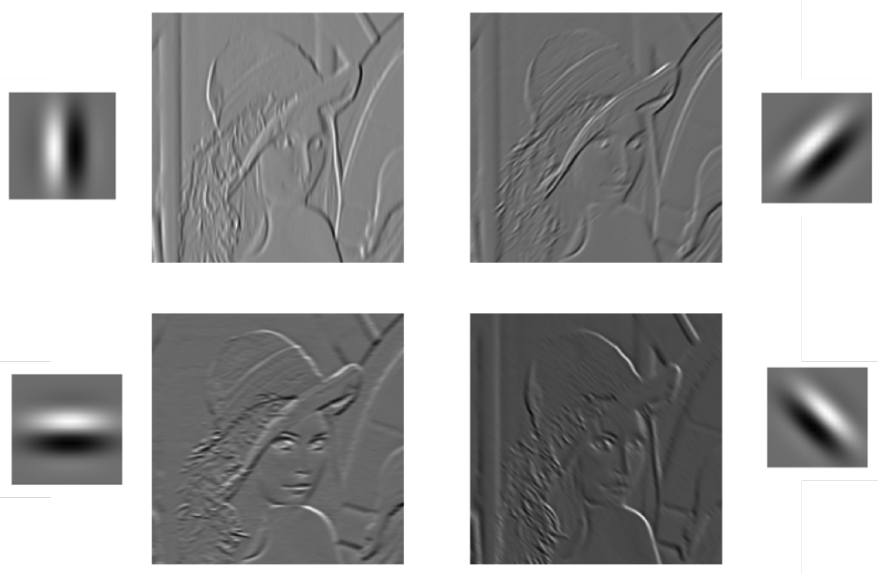

Figure 6.7: Gabor filter response using parallel array of STNOs for four different orientations of the filter.

difference of the image $F$ and the Gabor filter $G$ patterns on a pixel by pixel basis:

$$
\omega_{j}=\omega_{0}+\Delta \omega\left(F_{j}-G_{j}\right)
$$

As the oscillators evolve (Fig. 6.6), their instantaneous frequencies drift. If they synchronize, their final frequencies lock. They can also partially synchronize, with some oscillators remaining at different frequencies.

From Fig. 6.6, we can see the similarities between the FSK scheme and the parallel, electrically connected array of spin torque nano-oscillators. In the texture detection, the test and reference inputs are replaced by the image and Gabor vectors respectively. The difference between the two vectors will shift the frequency of oscillation from the central frequency, $\omega_{0}$. The oscillators then interact with each other via a common transmission line and synchronize when the frequencies are similar to each other. 


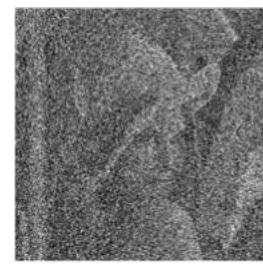

(a)

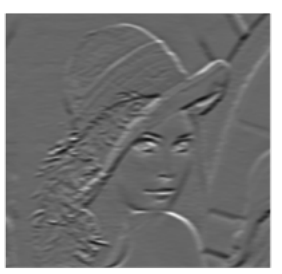

(b)

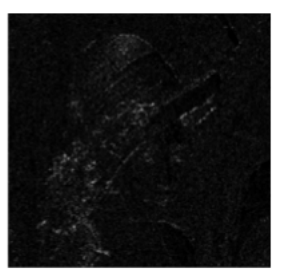

(c)

Figure 6.8: The effect of bandwidth $\Delta \omega$ on filter response. (a): $\Delta \omega / 2 \pi=10 \mathrm{GHz}$; (b): $\Delta \omega / 2 \pi=3.1 \mathrm{GHz}$ (optimum); (c): $\Delta \omega / 2 \pi=200 \mathrm{MHz}$

\subsubsection{Texture Detection}

Using the frequency shift keying scheme, we implemented the Gabor filter on a 64x64 image for four different filter orientation. The results are shown in Fig. 6.7. We see that for each Gabor filter, a different part of the image is emphasized while other parts are attentuated. These selected parts represent signature for various textures within the image.

One of the significant parameters within these simulations is the coupling bandwidth $\Delta \omega$. This number determines the scaling of the frequency based on the difference between the image and the Gabor filter. In Fig. 6.8, we show the effect of the bandwidth on the filtering process. When the bandwidth is too large (Fig. 6.8(a)), only a few oscillators synchronize, creating a noisy output signal. On the other hand, if the bandwidth is too small, then too many oscillators synchronize to the same value giving a near single-valued filtered image (Fig. 6.8(c)). However, there exist a range of bandwidths which allows for the right number of oscillators to synchronize to produce the Gabor filtered-image (Fig. 6.8(b)).

More work needs to be done to implement advanced techniques in pattern recognition such as rotation, scale transformations and edge detection which are part of a more realistic associative memory design. The use of oscillators for these purposes remains an active area of research which suggest that STNOs are a possible device implementation for these 

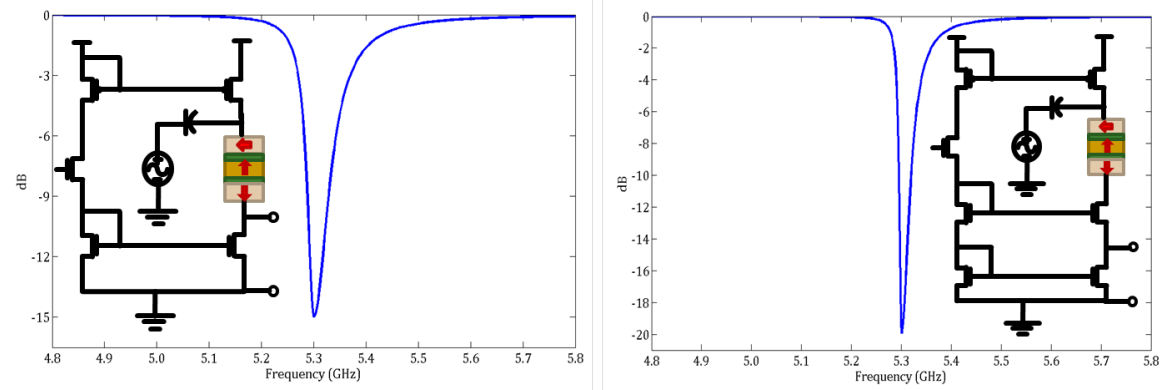

Figure 6.9: (a): An RF filter using STNO. A current mirror is used to set the DC current, which in turn, adjusts the resonating frequency. An input signal is applied to the STNO, which filters the frequencies near resonance. Depending on circuit topology, both bandpass and bandstop (pictured) can be implemented. (b): An RF filter using stacked current mirror. By stacking the footer of the current, we can increase the circuit impedance resulting in a deeper notch filter.

applications. Fabrication of these specialized spin torque oscillators also remains a challenge. However, with the recent advancements in PMA spin torque devices, DMTJs could be feasible in the near future. Finally, more simulation and modelling needs to be done on these STNO arrays to determine the robustness of the design to noise and process variations. These analyses will help refine and improve future circuit designs and architectures.

\subsection{Radio Frequency Circuits}

One of the most exciting aspects of the STNO devices is the possible application to systemon-chip (SoC) $[108,109]$. The nanoscale dimensions, coupled with the possibility of CMOS integration, low power, tunable frequency through magnetic fields and electrical currents, high quality factor $\mathrm{Q}(\geq 10,000)$ and the possible control of coupling opens many possibilities beyond the current state-of-the-art RF systems. One such RF application is as filters which take advantage of injection locking which has already been observed in STNOs [84]. Using a current mirror, we can create a circuit which allows the STNO to be tuned to a resonant 
frequency, and then apply an AC signal to the oscillator. For frequencies close to the center frequency, the STNO acts as either a bandpass or a band-reject filter depending on the circuit topology used. This allows us to do signal processing for a wide range of frequencies (1GHz-100GHz). This simple circuit acts as notch bandpass/bandstop filter with line widths of $10 \mathrm{MHz}$.

Although the STNOs can be used to filter narrow bandwidth signals, we are also interested in developing robust techniques to tune the filter strength and bandwidth. One of the ways to increase the filter strength is to improve the current mirror design used to set the STNO resonance oscillation. A stacked cascode structure (Figure 6.9) can be used to increase the circuit impedance, this strengthening the coupling of the STNO to the desired frequency. In our simulations, the stacked cascode structure produces a $5 \mathrm{~dB}$ improvement over the normal structure (Figure 6.9b).

Yet another technique to increase the filter strength is to use STNO synchronization. It has been shown that the oscillation power of the STNOs can be increased through the synchronization of multiple nano-oscillators $[76,88,110]$. In Figure 6.10, we use the same principle to boost the power of STNOs. The coherence creates a stronger resonance signal which helps to increase the filter strength. In Figure $6.10 \mathrm{~b}$, we show that a coupled STNO can enhance the filter strength by almost 10dB over a single STNO filter. However, the increase in strength begins to level out with more STNO since the oscillation power increases quadratically. The bandwidth of the STNO filter can also be adjusted through engineering the thermal stability of the free layer of the magnetic tunnel junction. The thermal stability determines the susceptibility of the free layer to noise. In general, spin torque oscillators are resilient to noise and thus create high Q-factor oscillations. However, the thermal stability 


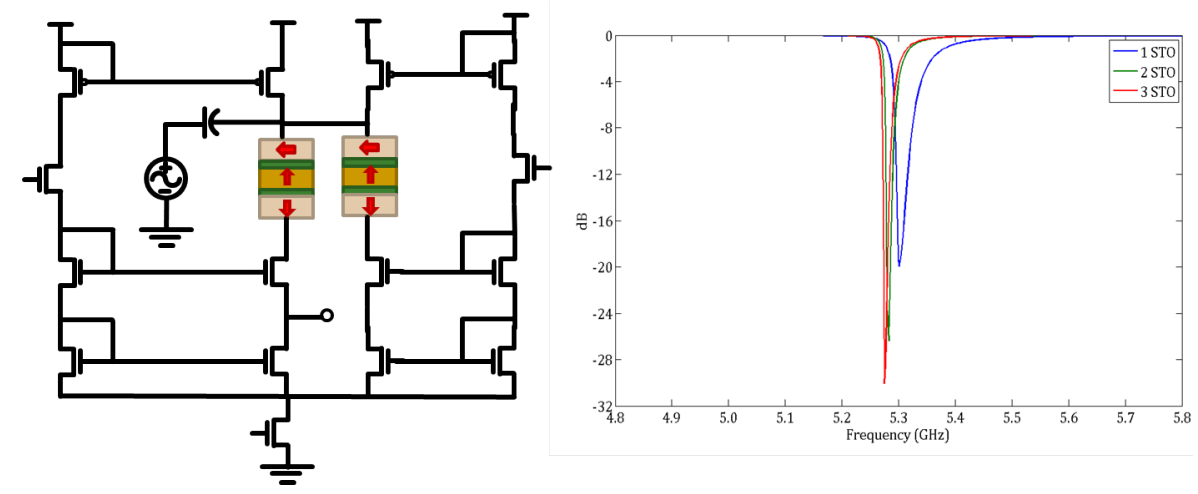

Figure 6.10: (a): A coupled STNO bandstop filter. The cascode current mirrors set the frequency of oscillation in the STNOs. When the frequencies are close together, the oscillators synchronize to produce a stronger filter. (b): The frequency analysis shows a deeper notch for more coupled STNOs. The increase in filter strength is superlinear $\left(\sim N^{2}\right)$

(Eqn. 1) can be exploited through device geometry and material properties to make the device more susceptible to noise.

$$
\Delta \approx \frac{H_{k} M_{s} V}{2 k_{B} T}
$$

where $H_{k}$ and $M_{s}$ are the free layer magnetic properties and $V$ is the volume of the layer. This effect allows other frequencies to be included in the STNO filter which broadens the filter bandwidth. However, there is a trade-off between filter bandwidth and the filter strength as the noise affects the integrity of the harmonic oscillations.

In addition to filters, there are also other RF applications which are actively being researched [111]. Because spin-torque devices exhibit an intrinsic instability leading to oscillation, these devices may also be exploited to realize self-oscillating RF mixers. Mixers are the central component of heterodyne receivers, which are the most important sensor architecture used for RF applications. Heterodyne receivers typically make use of a separate local oscillator with which the signal to be detected is mixed. This allows the power of the detected signal to be measured while maintaining phase coherence. STNO devices offer a 


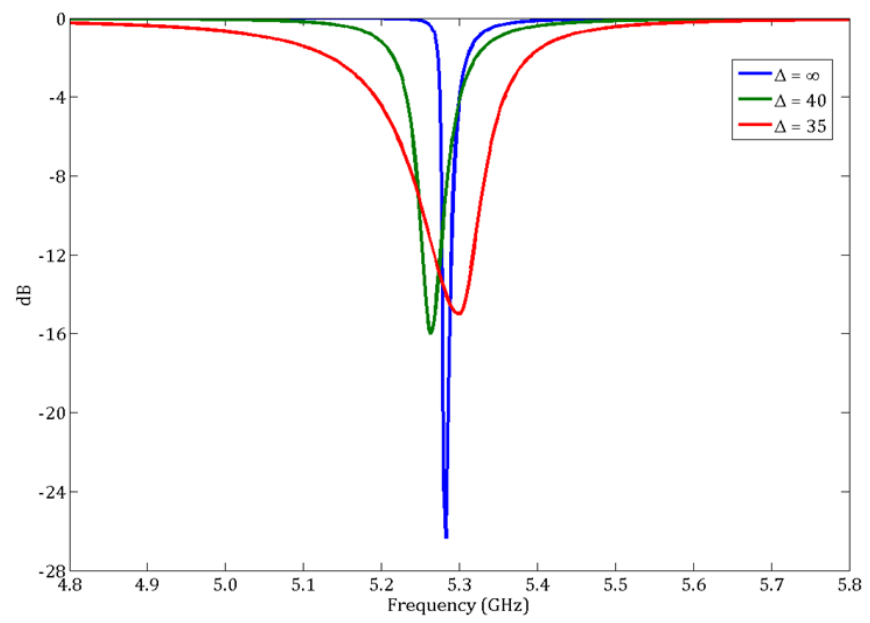

Figure 6.11: The effect of noise and thermal stability on STNO filters.

means of realizing self-oscillating mixers because the magnetic procession in these devices is inherently nonlinear. With the self-mixing characteristic of the device, the oscillation source and the mixer are combined in one device. In addition, the device is tunable via applied current or magnetic field. All these advantages make STNO device an intriguing choice for realizing $\mathrm{RF} /$ millimeter-wave tunable mixers. These preliminary results provide only a hint of some of the RF applications which are possible with nanoscale STNOs. The SoCs of the future can thus replace large power hungry active and passive CMOS devices with simple nanodevices with better characteristics. 


\section{Chapter 7}

\section{Conclusions}

The primary goal of this disseration has been to develop a unified framework in which we can describe the behavior of magnetic spintronic devices. In that respect, this work has created a Verilog-A model capable of modeling spintronic devices governed by the Landau-LifshitzGilbert equation. The development of this model has provided an invaluable scientific and engineering tool to not only explore the behavior spintronic devices, but also use it to design novel circuits and architecture for spin-based technologies.

We first extended this model to include multiferroic devices by developing a thermodynamic model for both ferroelectric and magnetostriction behavior in these special class of materials. Using this model we were able to show close correspondence between experiment and simulation.

In addition, we used this model to design a novel digital logic device known as the Reconfigurable Array of Magnetic Automata (RAMA). The Verilog-A multiferroic model not only exhibited binary switching, but also a third state which can be used for reconfiguration of logic gates similar to field programmable gate arrays (FPGAs). This work then proposed 
a logic architecture based on cellular automata computation. We developed a logic system based on majority gate like structures and a four-phased clocking mechanism which can realize any arbitrary logic. Therefore, RAMA can be an attractive future technology targeting ultra low-power applications. Yet another application of the Verilog-A model has been to simulate the behavior of spin-torque nano-oscillators (STNOs). After extending the model to include the effects of spin transfer torque, we were able to show some remarkable properties of this spin-based oscillator from frequency tunability to synchronization. This work not only validated the device model with experimental results, but also developed an analytical model which can be used to explain the precession generation and synchronization dynamics.

Using this Verilog-A model, we designed applications which take advantage of the synchronization behavior of STNOs. The first application was towards an oscillatory neurocomputer which can be used for pattern recognition. We further developed this recognition circuit to perform more complicated tasks such texture segmentation. Finally, this dissertation proposed using synchronized STNOs for tunable radio-frequency filters. A proof-of-concept demonstrated that STNOs have great flexibility and can be adjusted to provide narrow-band filtering with adjustable parameters such as frequency and bandwidth.

\subsection{Future Work}

In this dissertation, we have only scratched the surface of the possible applications using spintronic devices. There still exist several engineering challenges, such as fabrication and optimized integration of these devices with traditional MOSFET technologies. However, given the theoretical background, rapidly progressing experimental studies and similarities 
to systems in nature and other fields of study, spintronic devices are very promising for future conventional and non-conventional applications. Furthermore, their nanoscale size and compatibility with MOSFET technology opens the possibility of "More-than-Moore" type applications. Some possible future directions for the work presented in this dissertation are:

- Extending the Verilog-A model to include Fourier based LLG solver rather than macrospin model for capturing the behavior and structure of magnetic domains.

- Development of a near-memory computational device using multiferroic nanopillars for both nonvolatile memory and reconfigurable logic.

- Study of optimized synthesis and placement of MQCA gates for RAMA-based logic.

- Optimizing interconnect design within MQCA logic for better criss-crossing of "wires".

- Extending the transport model of spin transfer torque to modify Slonczewski's barrier model to Non-equilibrium Green Formalism (NEGF) for more accurate characterization of the magnetic tunnel junction.

- Modify the pattern recognition architecture for edge detection through Laplacian filters.

- Implement Hebbian learning using STNO based oscillatory neurocomputer.

- Study better methods of improving the output power of STNO through synchronization and amplification. 


\section{Appendix: Simulation Parameters}

All simulations were done using an HSPICE device model of spin torque nano-oscillators. This device model solves the Landau-Lifshitz-Gilbert-Slonczewski (LLGS) equations for a macrospin model. The material properties were extracted from experimental values for $\mathrm{Co}_{2} \mathrm{FeAl} / \mathrm{MgO} / \mathrm{Co}_{2} \mathrm{FeAl}$ MTJ structures. Some of the simulation properties are listed in

Table 1. We also used 45nm PTM transistor model [112].

Table 1: Simulation Parameters

\begin{tabular}{|c|c|}
\hline Material Parameter & Value \\
\hline Saturation Mag. $\left(M_{s}\right)$ & $1 \times 10^{6} \mathrm{~A} / \mathrm{m}$ \\
In-plane Anisotropy $\left(H_{\text {in }}\right)$ & $5 \times 10^{5} \mathrm{~J} / \mathrm{m}^{3}$ \\
Out-of-plane Anisotropy $\left(H_{\text {out }}\right)$ & $2 \times 10^{6} \mathrm{~J} / \mathrm{m}^{3}$ \\
Damping parameter $(\alpha)$ & 0.01 \\
Spin polarization $(P)$ & 0.6 \\
\hline
\end{tabular}




\section{Bibliography}

[1] C. Y. Sung. Post CMOS Nanoelectronics Research for the Next Generation Logic Switches. In International Symposium on VLSI Technology, Systems and Applications, 2008. VLSI-TSA 2008, pages 68-69, April 2008.

[2] W. Arden, M. Brillouët, P. Cogez, M. Graef, B. Huizing, and R. Mahnkopf. Morethan-Moore white paper. Version, 2:14, 2010.

[3] Eric D. Daniel, C. Denis Mee, and Mark H. Clark. Magnetic Recording: The First 100 Years. In Magnetic Recording: The First 100 Years, pages 48-49. John Wiley \& Sons, 1999. Google-Books-ID: 7WrCSCqMk5gC.

[4] S. A. Wolf, J. Lu, M. R. Stan, E. Chen, and D. M. Treger. The Promise of Nanomagnetics and Spintronics for Future Logic and Universal Memory. Proceedings of the IEEE, 98(12):2155-2168, December 2010.

[5] M. N. Baibich, J. M. Broto, A. Fert, F. Nguyen Van Dau, F. Petroff, P. Etienne, G. Creuzet, A. Friederich, and J. Chazelas. Giant Magnetoresistance of (001)Fe/(001)Cr Magnetic Superlattices. Phys. Rev. Lett., 61(21):2472-2475, November 1988 .

[6] G. Binasch, P. Grünberg, F. Saurenbach, and W. Zinn. Enhanced magnetoresistance in layered magnetic structures with antiferromagnetic interlayer exchange. Phys. Rev. B, 39(7):4828-4830, March 1989.

[7] W. Eerenstein, N. D. Mathur, and J. F. Scott. Multiferroic and magnetoelectric materials. Nature, 442(7104):759-765, August 2006.

[8] David J. Griffiths. Introduction to Quantum Mechanics. Cambridge University Press, August 2016.

[9] R. Shankar. Principles of Quantum Mechanics. Springer US, December 2013.

[10] B. D. Cullity and C. D. Graham. Introduction to Magnetic Materials. John Wiley \& Sons, October 2011.

[11] Charles Kittel. Introduction to Solid State Physics. Wiley, November 2004.

[12] T. L. Gilbert. A phenomenological theory of damping in ferromagnetic materials. IEEE Transactions on Magnetics, 40(6):3443-3449, November 2004. 
[13] NIST. OOMMF Project.

[14] muMAG Micromagnetics.

[15] Anurag Nigam, Clinton W. Smullen IV, Vidyabhushan Mohan, Eugene Chen, Sudhanva Gurumurthi, and Mircea R. Stan. Delivering on the promise of universal memory for spin-transfer torque RAM (STT-RAM). In Proceedings of the 17th IEEE/ACM international symposium on Low-power electronics and design, pages 121-126. IEEE Press, 2011.

[16] Kamaram Munira. Nanomagnetic Memory and Logic: Energy-delay-reliability Tradeoff. PhD thesis, University of Virginia, University of Virginia, December 2012.

[17] C. Nan, M. I. Bichurin, S. Dong, D. Viehland, and G. Srinivasan. Multiferroic magnetoelectric composites: Historical perspective, status, and future directions. Journal of Applied Physics, 103(3):031101, February 2008.

[18] Nicola A. Hill. Why Are There so Few Magnetic Ferroelectrics? J. Phys. Chem. B, 104(29):6694-6709, July 2000.

[19] J. F. Scott. Data storage: Multiferroic memories. Nature Materials, 6(4):256, April 2007.

[20] W. Prellier, M. P. Singh, and P. Murugavel. The single-phase multiferroic oxides: from bulk to thin film. J. Phys.: Condens. Matter, 17(30):R803, 2005.

[21] Sang-Wook Cheong and Maxim Mostovoy. Multiferroics: a magnetic twist for ferroelectricity. Nat Mater, 6(1):13-20, January 2007.

[22] Ce-Wen Nan. Magnetoelectric effect in composites of piezoelectric and piezomagnetic phases. Phys. Rev. B, 50(9):6082-6088, September 1994.

[23] R. E. Newnham, D. P. Skinner, and L. E. Cross. Connectivity and piezoelectricpyroelectric composites. Materials Research Bulletin, 13(5):525-536, May 1978.

[24] F. Zavaliche, H. Zheng, L. Mohaddes-Ardabili, S. Y. Yang, Q. Zhan, P. Shafer, E. Reilly, R. Chopdekar, Y. Jia, P. Wright, D. G. Schlom, Y. Suzuki, and R. Ramesh. Electric Field-Induced Magnetization Switching in Epitaxial Columnar Nanostructures. Nano Letters, 5(9):1793-1796, September 2005.

[25] N. Hur, S. Park, P. A. Sharma, J. S. Ahn, S. Guha, and S.-W. Cheong. Electric polarization reversal and memory in a multiferroic material induced by magnetic fields. Nature, 429(6990):392-395, May 2004.

[26] T. Lottermoser, T. Lonkai, U. Amann, D. Hohlwein, J. Ihringer, and M. Fiebig. Magnetic phase control by an electric field. Nature, 430(6999):541-544, July 2004.

[27] S. Ren, R. M. Briber, and M. Wuttig. Diblock copolymer based self-assembled nanomagnetoelectric. Applied Physics Letters, 93(17):173507, October 2008. 
[28] J. K. W. Yang, Y. S. Jung, J. B. Chang, R. A. Mickiewicz, A. Alexander-Katz, C. A. Ross, and K. K. Berggren. Complex self-assembled patterns using sparse commensurate templates with locally varying motifs. Nature Nanotechnology, 5(4):256-260, April 2010.

[29] A. F. Devonshire. Theory of ferroelectrics. Advances in Physics, 3(10):85-130, April 1954.

[30] P. Chandra and P. B. Littlewood. A Landau Primer for Ferroelectrics. September 2006.

[31] Wenwu Cao. Constructing Landau-Ginzburg-Devonshire Type Models for Ferroelectric Systems Based on Symmetry. Ferroelectrics, 375(1):28-39, December 2008.

[32] Earl Callen and Herbert B. Callen. Magnetostriction, Forced Magnetostriction, and Anomalous Thermal Expansion in Ferromagnets. Phys. Rev., 139(2A):A455-A471, July 1965.

[33] E. W. Lee. Magnetostriction and Magnetomechanical Effects. Rep. Prog. Phys., 18(1):184, 1955.

[34] R. D. James and Manfred Wuttig. Magnetostriction of martensite. Philosophical Magazine A, 77(5):1273-1299, May 1998.

[35] H. Zheng, J. Wang, S. E. Lofland, Z. Ma, L. Mohaddes-Ardabili, T. Zhao, L. Salamanca-Riba, S. R. Shinde, S. B. Ogale, F. Bai, and others. Multiferroic $\mathrm{BaTiO}_{3}-\mathrm{CoFe}_{2} \mathrm{O}_{4}$ nanostructures. Science, 303(5658):661-663, 2004.

[36] Khalid Ashraf and Sayeef Salahuddin. Effect of anti-ferromagnet surface moment density on the hysteresis properties of exchange coupled antiferromagnet-ferromagnet systems: The case of bismuth-ferrite. Journal of Applied Physics, 111(10):103904, May 2012.

[37] John Von Neumann. Theory of Self-Reproducing Automata. January 1966.

[38] John Conway. Game of Life. Scientific American, (223), October 1970.

[39] P. Douglas Tougaw and Craig S. Lent. Logical Devices Implemented Using Quantum Cellular Automata. ResearchGate, 75(3):1818-1825, March 1994.

[40] R. P. Cowburn and M. E. Welland. Room Temperature Magnetic Quantum Cellular Automata. Science, 287(5457):1466-1468, February 2000.

[41] M. Kabir, M. R. Stan, S. A. Wolf, R. B. Comes, and J. Lu. RAMA: A Self-assembled Multiferroic Magnetic QCA for Low Power Systems. In Proceedings of the 21st Edition of the Great Lakes Symposium on Great Lakes Symposium on VLSI, GLSVLSI '11, pages 25-30, New York, NY, USA, 2011. ACM. 
[42] M. R. Stan, M. Kabir, J. Lu, and S. Wolf. Self-assembled multiferroic magnetic QCA structures for low power systems. In 2012 IEEE International Symposium on Circuits and Systems, pages 2525-2528, May 2012.

[43] J. Huang, M. Momenzadeh, L. Schiano, M. Ottavi, and F. Lombardi. Tile-based QCA Design Using Majority-like Logic Primitives. J. Emerg. Technol. Comput. Syst., 1(3):163-185, October 2005.

[44] J. Huang, M. Momenzadeh, L. Schiano, and F. Lombardi. Simulation-based design of modular QCA Circuits. In 5th IEEE Conference on Nanotechnology, 2005., pages 533-536 vol. 2, July 2005.

[45] D. Berzon and T. J. Fountain. A memory design in QCAs using the SQUARES formalism. In Proceedings Ninth Great Lakes Symposium on VLSI, pages 166-169, March 1999.

[46] K. Bernstein, R. K. Cavin, W. Porod, A. Seabaugh, and J. Welser. Device and Architecture Outlook for Beyond CMOS Switches. Proceedings of the IEEE, 98(12):21692184, December 2010.

[47] R. Meservey and P. M. Tedrow. Spin-polarized electron tunneling. Physics Reports, 238(4):173-243, March 1994.

[48] Evgeny Y. Tsymbal, Oleg N. Mryasov, and Patrick R. LeClair. Spin-dependent tunnelling in magnetic tunnel junctions. J. Phys.: Condens. Matter, 15(4):R109, February 2003.

[49] G. D. Fuchs, N. C. Emley, I. N. Krivorotov, P. M. Braganca, E. M. Ryan, S. I. Kiselev, J. C. Sankey, D. C. Ralph, R. A. Buhrman, and J. A. Katine. Spin-transfer effects in nanoscale magnetic tunnel junctions. Applied Physics Letters, 85(7):1205, 2004.

[50] Albert Fert. Nobel Lecture: Origin, development, and future of spintronics. Rev. Mod. Phys., 80(4):1517-1530, December 2008.

[51] Peter A. Grünberg. Nobel Lecture: From spin waves to giant magnetoresistance and beyond. Rev. Mod. Phys., 80(4):1531-1540, December 2008.

[52] R. Meservey, P. M. Tedrow, and Peter Fulde. Magnetic Field Splitting of the Quasiparticle States in Superconducting Aluminum Films. Phys. Rev. Lett., 25(18):12701272, November 1970.

[53] E. Y. Tsymbal and D. G. Pettifor. Perspectives of giant magnetoresistance. In Henry Ehrenreich and Frans Spaepen, editor, Solid State Physics, volume Volume 56, pages 113-237. Academic Press, 2001.

[54] J. Bardeen, L. N. Cooper, and J. R. Schrieffer. Theory of Superconductivity. Phys. Rev., 108(5):1175-1204, December 1957. 
[55] B. D. Josephson. Possible new effects in superconductive tunnelling. Physics Letters, 1:251-253, July 1962.

[56] M. Julliere. Tunneling between ferromagnetic films. Physics Letters A, 54(3):225-226, September 1975.

[57] W. Patrick McCray. MBE deserves a place in the history books. Nat Nano, 2(5):259261, May 2007.

[58] T. Valet and A. Fert. Theory of the perpendicular magnetoresistance in magnetic multilayers. Physical Review B, 48:7099-7113, September 1993.

[59] T. Miyazaki and N. Tezuka. Giant magnetic tunneling effect in $\mathrm{Fe} / \mathrm{Al}_{2} \mathrm{O}_{3} / \mathrm{Fe}$ junction. Journal of Magnetism and Magnetic Materials, 139(3):L231-L234, 1995.

[60] D. L. Zhang, X. G. Xu, Y. Wu, J. Miao, and Y. Jiang. Effect of nano-oxide layers on giant magnetoresistance in pseudo-spin-valves using Co2feal electrodes. Journal of Magnetism and Magnetic Materials, 323(5):631-634, March 2011.

[61] J. S. Moodera, Lisa R. Kinder, Terrilyn M. Wong, and R. Meservey. Large Magnetoresistance at Room Temperature in Ferromagnetic Thin Film Tunnel Junctions. Phys. Rev. Lett., 74(16):3273-3276, April 1995.

[62] J. C. Slonczewski. Conductance and exchange coupling of two ferromagnets separated by a tunneling barrier. Phys. Rev. B, 39(10):6995-7002, April 1989.

[63] J. C. Slonczewski. Current-driven excitation of magnetic multilayers. Journal of Magnetism and Magnetic Materials, 159:L1-L7, June 1996.

[64] L. Berger. Emission of spin waves by a magnetic multilayer traversed by a current. Phys. Rev. B, 54(13):9353-9358, October 1996.

[65] L. Berger. Influence of current leads on critical current for spin precession in magnetic multilayers. Journal of Magnetism and Magnetic Materials, 278(1-2):185-194, July 2004.

[66] M. Tsoi, A. G. M. Jansen, J. Bass, W.-C. Chiang, M. Seck, V. Tsoi, and P. Wyder. Excitation of a magnetic multilayer by an electric current. Physical Review Letters, 80(19):4281, 1998.

[67] J. Z Sun. Current-driven magnetic switching in manganite trilayer junctions. Journal of Magnetism and Magnetic Materials, 202(1):157-162, June 1999.

[68] J. A. Katine, F. J. Albert, R. A. Buhrman, E. B. Myers, and D. C. Ralph. CurrentDriven Magnetization Reversal and Spin-Wave Excitations in $\mathrm{Co} / \mathrm{Cu} / \mathrm{Co}$ Pillars. Phys. Rev. Lett., 84(14):3149-3152, April 2000. 
[69] E. Chen, D. Apalkov, Z. Diao, A. Driskill-Smith, D. Druist, D. Lottis, V. Nikitin, X. Tang, S. Watts, S. Wang, S.A. Wolf, A. W. Ghosh, J.W. Lu, S. J. Poon, M. Stan, W.H. Butler, S. Gupta, C. K A Mewes, T. Mewes, and P.B. Visscher. Advances and Future Prospects of Spin-Transfer Torque Random Access Memory. IEEE Transactions on Magnetics, 46(6):1873-1878, June 2010.

[70] C.W. Smullen, V. Mohan, A. Nigam, S. Gurumurthi, and M.R. Stan. Relaxing non-volatility for fast and energy-efficient STT-RAM caches. In 2011 IEEE 17th International Symposium on High Performance Computer Architecture (HPCA), pages 50-61, February 2011.

[71] S. I. Kiselev, J. C. Sankey, I. N. Krivorotov, N. C. Emley, R. J. Schoelkopf, R. A. Buhrman, and D. C. Ralph. Microwave oscillations of a nanomagnet driven by a spin-polarized current. Nature, 425(6956):380-383, September 2003.

[72] A. V. Nazarov, H. M. Olson, H. Cho, K. Nikolaev, Z. Gao, S. Stokes, and B. B. Pant. Spin transfer stimulated microwave emission in $\mathrm{MgO}$ magnetic tunnel junctions. Applied Physics Letters, 88(16):162504, 2006.

[73] W. Rippard, M. Pufall, and S. Russek. Comparison of frequency, linewidth, and output power in measurements of spin-transfer nanocontact oscillators. Physical Review B, 74(22), December 2006.

[74] W. Rippard, M. Pufall, S. Kaka, S. Russek, and T. Silva. Direct-Current Induced Dynamics in $\mathrm{Co}_{90} \mathrm{Fe}_{10} / \mathrm{Ni}_{80} \mathrm{Fe}_{20}$ Point Contacts. Physical Review Letters, 92(2), January 2004.

[75] S. Kaka, M. R. Pufall, W. H. Rippard, T. J. Silva, S. E. Russek, and J. A. Katine. Mutual phase-locking of microwave spin torque nano-oscillators. Nature, 437(7057):389-392, September 2005.

[76] S. E. Russek, W. H. Rippard, T. Cecil, and R. Heindl. Spin-Transfer Nano-Oscillators. In Handbook of Nanophysics: Functional Nanomaterials, volume 38, pages 1-22. CRC Press, September 2010.

[77] T. Shibata, Renyuan Zhang, S.P. Levitan, D.E. Nikonov, and G.I. Bourianoff. CMOS supporting circuitries for nano-oscillator-based associative memories. In 2012 13th International Workshop on Cellular Nanoscale Networks and Their Applications (CNNA), pages 1-5, August 2012.

[78] S.P. Levitan, Yan Fang, D.H. Dash, T. Shibata, D.E. Nikonov, and G.I. Bourianoff. Non-Boolean associative architectures based on nano-oscillators. In 2012 13th International Workshop on Cellular Nanoscale Networks and Their Applications (CNNA), pages 1-6, August 2012.

[79] Andrei Slavin and Vasil Tiberkevich. Nonlinear Auto-Oscillator Theory of Microwave Generation by Spin-Polarized Current. IEEE Transactions on Magnetics, 45(4):18751918, April 2009. 
[80] Z. Li, Y. Li, and S. Zhang. Dynamic magnetization states of a spin valve in the presence of dc and ac currents: Synchronization, modification, and chaos. Physical Review B, 74(5), August 2006.

[81] Pierre Baldi and Ronny Meir. Computing with arrays of coupled oscillators: An application to preattentive texture discrimination. Neural Computation, 2(4):458-471, 1990.

[82] K. Wiesenfeld, P. Colet, and S. H. Strogatz. Frequency locking in Josephson arrays: connection with the Kuramoto model. Physical Review E, 57(2):1563, 1998.

[83] MK Stephen Yeung and Steven H. Strogatz. Time delay in the Kuramoto model of coupled oscillators. Physical Review Letters, 82(3):648, 1999.

[84] W. Rippard, M. Pufall, S. Kaka, T. Silva, S. Russek, and J. Katine. Injection Locking and Phase Control of Spin Transfer Nano-oscillators. Physical Review Letters, 95(6), August 2005.

[85] S. Sani, J. Persson, S. M. Mohseni, Ye Pogoryelov, P. K. Muduli, A. Eklund, G. Malm, M. Kll, A. Dmitriev, and J. kerman. Mutually synchronized bottom-up multi-nanocontact spintorque oscillators. Nat Commun, 4, November 2013.

[86] F. B. Mancoff, N. D. Rizzo, B. N. Engel, and S. Tehrani. Phase-locking in doublepoint-contact spin-transfer devices. Nature, 437(7057):393-395, September 2005.

[87] S. Urazhdin, P. Tabor, V. Tiberkevich, and A. Slavin. Fractional Synchronization of Spin-Torque Nano-Oscillators. Phys. Rev. Lett., 105(10):104101, August 2010.

[88] J. Grollier, V. Cros, and A. Fert. Synchronization of spin-transfer oscillators driven by stimulated microwave currents. Physical Review B, 73(6), February 2006.

[89] B. Georges, J. Grollier, V. Cros, and A. Fert. Impact of the electrical connection of spin transfer nano-oscillators on their synchronization: an analytical study. Applied Physics Letters, 92(23):232504, June 2008.

[90] V. Tiberkevich, A. Slavin, E. Bankowski, and G. Gerhart. Phase-locking and frustration in an array of nonlinear spin-torque nano-oscillators. Applied Physics Letters, 95(26):262505, December 2009.

[91] D. C. Ralph and M. D. Stiles. Spin transfer torques. Journal of Magnetism and Magnetic Materials, 320(7):1190-1216, April 2008.

[92] Mehdi Kabir and Mircea Stan. Computing with Hybrid CMOS/STO Circuits. In Proceedings of the The 51st Annual Design Automation Conference on Design Automation Conference, DAC '14, pages 73:1-73:6, New York, NY, USA, 2014. ACM.

[93] Mehdi Kabir and Mircea Stan. Synchronized Spin Torque Nano-Oscillators: From Theory to Applications. In Weisheng Zhao and Guillaume Prenat, editors, Spintronicsbased Computing, pages 231-250. Springer International Publishing, Cham, 2015. 
[94] J. W. Lu, E. Chen, M. Kabir, M. R. Stan, and S. A. Wolf. Spintronics technology: past, present and future. International Materials Reviews, 61(7):456-472, October 2016.

[95] Yoshiki Kuramoto. Chemical Oscillations, Waves, and Turbulence. Courier Dover Publications, 2003.

[96] Arkady Pikovsky. Synchronization: A Universal Concept in Nonlinear Sciences. Cambridge University Press, April 2003.

[97] J. Persson, Yan Zhou, and Johan Akerman. Phase-locked spin torque oscillators: Impact of device variability and time delay. Journal of Applied Physics, 101(9):09A503, 2007.

[98] A.K. Jain, K.K. Likharev, J.E. Lukens, and J.E. Sauvageau. Mutual phase-locking in Josephson junction arrays. Physics Reports, 109(6):309-426, July 1984.

[99] A.N. Slavini and P. Kabos. Approximate theory of microwave generation in a currentdriven magnetic nanocontact magnetized in an arbitrary direction. IEEE Transactions on Magnetics, 41(4):1264-1273, April 2005.

[100] Frank C. Hoppensteadt and Eugene M. Izhikevich. Oscillatory Neurocomputers with Dynamic Connectivity. Phys. Rev. Lett., 82(14):2983-2986, April 1999.

[101] Z. Zeng, G. Finocchio, B. Zhang, P. K. Amiri, J. A. Katine, I. N. Krivorotov, Y. Huai, J. Langer, B. Azzerboni, K. L. Wang, and H. Jiang. Ultralow-current-density and bias-field-free spin-transfer nano-oscillator. Sci. Rep., 3, March 2013.

[102] T. Devolder, A. Meftah, K. Ito, J. A. Katine, P. Crozat, and C. Chappert. Spin transfer oscillators emitting microwave in zero applied magnetic field. Journal of Applied Physics, 101(6):063916, March 2007.

[103] I. Krivorotov, D. Berkov, N. Gorn, N. Emley, J. Sankey, D. Ralph, and R. Buhrman. Large-amplitude coherent spin waves excited by spin-polarized current in nanoscale spin valves. Physical Review B, 76(2), July 2007.

[104] G. Feng, Sebastiaan van Dijken, J. F. Feng, J. M. D. Coey, T. Leo, and David J. Smith. Annealing of $\mathrm{CoFeB} / \mathrm{MgO}$ based single and double barrier magnetic tunnel junctions: Tunnel magnetoresistance, bias dependence, and output voltage. Journal of Applied Physics, 105(3):033916, February 2009.

[105] Anil K. Jain and Farshid Farrokhnia. Unsupervised Texture Segmentation Using Gabor Filters. Pattern Recogn., 24(12):1167-1186, December 1991.

[106] John G. Daugman. Two-dimensional spectral analysis of cortical receptive field profiles. Vision Research, 20(10):847-856, 1980.

[107] D. E. Nikonov, I. A. Young, and G. I. Bourianoff. Convolutional networks for image processing by coupled oscillator arrays. arXiv preprint arXiv:1409.4469, 2014. 
[108] M.R. Stan, M. Kabir, Jiwei Lu, and S. Wolf. Nano-pattemed coupled spin torque nano oscillator (STNO) arrays-A potentially disruptive multipurpose nanotechnology. In New Circuits and Systems Conference (NEWCAS), 2013 IEEE 11th International, pages 1-4, June 2013.

[109] M.R. Stan, M. Kabir, S. Wolf, and Jiwei Lu. Spin torque nano oscillators as key building blocks for the Systems-on-Chip of the future. In 2014 IEEE/ACM International Symposium on Nanoscale Architectures (NANOARCH), pages 37-38, July 2014.

[110] B. Razavi. A study of injection locking and pulling in oscillators. IEEE Journal of Solid-State Circuits, 39(9):1415-1424, September 2004.

[111] N. Locatelli, V. Cros, and J. Grollier. Spin-torque building blocks. Nature Materials, 13(1):11-20, December 2013.

[112] Arizona State University. Predictive Technology Model (PTM). 